\title{
Tests and Problems of the Standard Model in Cosmology
}

\author{
Martín López-Corredoira
}

\begin{abstract}
The main foundations of the standard 1 CDM model of cosmology are that: 1) The redshifts of the galaxies are due to the expansion of the Universe plus peculiar motions; 2) The cosmic microwave background radiation and its anisotropies derive from the high energy primordial Universe when matter and radiation became decoupled; 3) The abundance pattern of the light elements is explained in terms of primordial nucleosynthesis; and 4) The formation and evolution of galaxies can be explained only in terms of gravitation within a inflation+dark matter+dark energy scenario. Numerous tests have been carried out on these ideas and, although the standard model works pretty well in fitting many observations, there are also many data that present apparent caveats to be understood with it. In this paper, I offer a review of these tests and problems, as well as some examples of alternative models.
\end{abstract}

Keywords Cosmology · Observational cosmology · Origin, formation, and abundances of the elements · dark matter · dark energy · superclusters and large-scale structure of the Universe

PACS 98.80.-k $\cdot 98.80 . \mathrm{E} \cdot 98.80 . \mathrm{Ft} \cdot 95.35 .+\mathrm{d} \cdot 95.36 .+\mathrm{x} \cdot$ 98.65.Dx

Mathematics Subject Classification (2000) 85A40 $\cdot 85-03$

\section{Introduction}

There is a dearth of discussion about possible wrong statements in the foundations of standard cosmology (the "Big Bang" hypothesis in the present-day version of $\Lambda \mathrm{CDM}$, i.e. model which includes cold dark matter and the $\Lambda$ term

Instituto de Astrofísica de Canarias, E-38205 La Laguna, Tenerife, Spain

Departamento de Astrofísica, Universidad de La Laguna, E-38206 La Laguna, Tenerife, Spain

Tel.: +34-922-605264

Fax: +34-922-605210

E-mail: martinlc@iac.es 
in the energy, standing for dark energy or quintessence). Most cosmologists are pretty sure that they have the correct theory, and that they do not need to think about possible major errors in the basic notions of their standard theory. Most works in cosmology are dedicated to refining small details of the standard model and do not worry about the foundations. There is still, however, a significant number of results in isolated and disconnected papers that are usually ignored by the leading cosmologists, and that are more challenging and critical of the standard model. My intention here is to bring together many of those heretical papers in order to help the more open-minded cosmologists to search the bibliography on tests and problems of the standard model.

In this paper, I will critically review the most important assumptions of the standard cosmological scenarios:

- The redshifts of the galaxies are due to the expansion of the Universe.

- The cosmic microwave background radiation and its anisotropies come from the high energy primordial Universe.

- The abundance pattern of the light elements is to be explained in terms of the primordial nucleosynthesis.

- The formation and evolution of galaxies can only be explained in terms of gravitation in the cold dark matter theory of an expanding Universe.

Some observations will be discussed or rediscussed in order to show that these facts were not strictly proven in some cases, but also, in other cases to show the solidity of the standard theory against certain tests.

There are many alternative theories. However, the purpose of this review is not to analyze the different theories, but to concentrate on the observational facts. In Ref. 1] I have reviewed theoretical ideas in cosmology that differ from the standard "Big Bang". It would be interesting to extend this brief analysis to a wider literature survey, but that is a vast task well beyond the scope of the present review. Another review paper would be necessary to compile these alternative ideas and complete the present review. Here, I focus mainly in the compilation of facts and their interpretations.

Cosmologists do not usually work within the framework of alternative cosmologies because they feel that these are not at present as competitive as the standard model. Certainly, they are not so developed, and they are not so developed because cosmologists do not work on them. It is a vicious circle. The fact that most cosmologists do not pay alternative theories any attention and only dedicate their research time to the standard model is to a great extent due to the sociological phenomenon known as the "snowball effect" or "groupthink" 1. This restricted view is unfair; therefore, I consider it appropriate to open the door here to further discussion of the results of the fundamental observations of cosmology.

It is not my purpose to defend a particular theory against the standard cosmology. All theories have their own problems. Only the problems of the standard Big Bang theory are put forward here, but many more affect the alternative models. Not all the problems and all the papers are considered since the literature is too vast in this broad topic. 
I have chosen to review the general aspects of the foundations of cosmology as a whole instead of certain branches of it because I am interested in expressing the caveats and open questions as a whole in order to extract global conclusions on cosmology as a whole. It may also be that some of the caveats presented are no longer caveats, or that some of the observational measurements are not correct. Warning: I review only certain critical papers, and in a few cases I discuss them, but I take no responsibility for their contents. In most cases, I collect the information given by the different papers without any further research; I am conscious in any case that most of the most critical papers need further analysis in the problems they posit before reaching a firm conclusion on whether the standard model is correct or not. My own position is also neutral, I express no opinion on whether the standard cosmology is correct or not.

\section{Redshift and expansion}

2.1 Is a static model theoretically impossible?

A static model is usually rejected by most cosmologists. However, from a purely theoretical point of view, the representation of the Cosmos as Euclidean and static is not excluded. Both expanding and static space are possible for the description of the Universe.

Before Einstein and the rise of Riemannian and other non-Euclidean geometries to the stage of physics, there were attempts to describe the known Universe in terms of Euclidean geometry, but with the problem of justifying a stable equilibrium. Within a relativistic context, Einstein 2 proposed a static model including a cosmological constant, his biggest blunder according to himself. This model still has problems in guaranteeing stability, but it might be solved somehow. Narlikar \& Arp 3 solve it within some variation of the HoyleNarlikar conformal theory of gravity, in which small perturbations of the flat Minkowski spacetime would lead to small oscillations about the line element rather than to a collapse. Boehmer et al. 4] analyze the stability of the Einstein static universe by considering homogeneous scalar perturbations in the context of $f(R)$ modified theories of gravity, and it is found that a stable Einstein cosmos with a positive cosmological constant is possible. Other authors solve the stability problem with the variation of fundamental constants [5, 6]. Another idea by Van Flandern[7] is that hypothetical gravitons responsible for the gravitational interaction have a finite cross-sectional area, so that they can only travel a finite distance, however great, before colliding with another graviton. So the range of the force of gravity would necessarily be limited in this way and collapse is avoided.

The very concept of space expansion has its own problems [8,9. Curved geometry (general relativity and its modifications) has no conservation of the energy-momentum of the gravity field. However, Minkowski space follows the conservation of energy-momentum of the gravitational field. One 
approach with a material tensor field in Minkowski space is given in Feynman's gravitation[10, where the space is static but matter and fields can be expanding in a static space. Also worth mentioning is a model related to modern relativistic and quantum field theories of basic fundamental interactions (strong, weak, electromagnetic): the relativistic field gravity theory and fractal matter distribution in static Minkowski space[11.

Olber's paradox for an infinite Universe also needs subtle solutions, but extinction, absorption, and re-emission of light, fractal distribution of density, and the mechanism which itself produces the redshift of the galaxies might have something to do with its solution. These are old questions discussed in many classical books on cosmology (e.g., [12, ch. 3) and do not warrant further discussion here.

\subsection{Does redshift mean expansion?}

Lemaître 13 in 1927 and later Hubble 14 in 1929 established the redshift $(z)-$ apparent magnitude relation of the galaxies, which gave an observational clue that the Universe is expanding. Hubble was cautious in suggesting this interpretation, but the following generations of cosmologists became pretty sure that the redshift of the galaxies following Hubble's law is a definitive proof of expansion. This was due mainly to the absence of a good theory that explains the possible phenomenological fact of alternative proposals. General relativity provided an explanation for the cosmological expansion, while alternative proposals were not supported by any well-known orthodox theory. The expansion was preferred and the phenomenological approaches which were not supported by present-day theory would be doomed to be forgotten. This position would be right if our physics represented all the phenomena in the Universe, but from a deductive-empiricist point of view we should deduce theories from the observations, and not the opposite.

\subsection{Alternative redshift theories}

There are other mechanisms that produce redshift [15, 16 apart from space expansion or the Doppler effect. A bibliographical catalogue with hundreds of references before the ' 80 s to 17 classes of phenomenologies with untrivial redshifts is collected by Reboul[17](Sect. 1), the main idea being the "tired light" scenario.

A tired light scenario assumes that the photon loses energy owing to some unknown photon-matter process or photon-photon interaction, when it travels some distance: the distance is long if we consider all the intergalactic space between the object and the earth, or short, for instance taking into consideration only the coronae enveloping the object. Indeed, it is not so much a theory as a possible phenomenological approach to explain the loss of the energy of the photons in a putative static Universe that could be explained by 
different theories. There are several hypothetical theories which can produce this "tired light" effect. The idea of loss of energy of the photon in the intergalactic medium was first suggested in 1929 by Zwicky[18 and was defended by him for a long time. Nernst in 1937 had developed a model which assumed that radiation was being absorbed by luminiferous ether. As late as the midtwentieth century, Zwicky[19] maintained that the hypothesis of tired light was viable. But there are two problems 15: 1) the $\phi$-bath smears out the coherence of the radiation from the source, and so all images of distant objects would look blurred if intergalactic space produced scattering, something that is incompatible with present-day observations [20]);2) the scattering effect and the consequent loss of energy would be frequency dependent, which is again incompatible with what we observe in galaxies 21]22] (S 4.1.1). Nonetheless, there are proposed solutions to these problems, as we will see in the following paragraphs.

Vigier 23 proposed a mechanism in which the vacuum behaves like a stochastic covariant superfluid aether whose excitations can interfere with the propagation of particles or light waves through it in a dissipative way. This avoids the two former difficulties of blurring and frequency dependence.

The photon-Raman interactions with atomic hydrogen [24,25] also explains shifts that emulate the Doppler effect with light-matter interaction that does not blur the images. Or the redshift may be produced by a surface plasma that is undergoing rapid radial expansion giving rise to population inversion and laser action in some atomic species [26].

The justification of the shift in photon frequency in a low density plasma could also come from quantum effects derived from standard quantum electrodynamics [27. According to Paul Marmet and Grote Reber (a co-initiator of radio astronomy), quantum mechanics indicates that a photon gives up a tiny amount of energy as it collides with an electron, but its trajectory does not change 28] (appendix); this is called plasma redshift 29, 30, 31]. This mechanism also avoids blurring and scattering. In another model 32 the photon is viewed as an electromagnetic wave whose electric field component causes oscillations in deep space free electrons, which then reradiate energy from the photon, causing a redshift. This new theoretical model is fundamentally different from Compton scattering, and therefore avoids any problems associated with Compton scattering, such as image blurring. Potentially, this effect could explain the high redshifts of apparently nearby QSOs (Quasi Stellar Objects), since light travelling through the outer atmosphere of the QSO could be redshifted before leaving it. In order to explain galactic redshifts with long travel distances in the scattering, the density in the intergalactic medium should be $10^{4}$ atoms $/ \mathrm{m}^{3}$, which is much higher than the density that is normally accepted $\left(\sim 10^{-1}\right.$ atoms $/ \mathrm{m}^{3}$ ). However, the disagreement in the density of the intergalactic medium is not necessarily a caveat in the hypothesis since our knowledge of the intergalactic medium is very poor, and there is also the possibility that intergalactic space is not so empty of baryonic matter.

The dynamic multiple scattering theory is also very interesting as a possible tired light mechanism. Results in statistical optics 33, 34] have shown 
that the shift in frequency of spectral lines is produced when light passes through a turbulent (or inhomogeneous) medium, owing to multiple scattering effects. The new frequency of the line is proportional to the old one, the redshift does not depend on the incident frequency 34. Redshift can also be explained in terms of non-linear optics, which assumes that the harmonic oscillator model of light has to be extended somewhat by an extremely small anharmonic contribution 35.

The redshift might be due to a tired-light gravitational interaction of wave packets with curved spacetime 36, 37, 38. The result is a redshift that is proportional to distance and to the square root of the density. Curvature pressure results from the non-geodesic motion of charged particles in the gravitational interaction.

Apart from the standard gravitational redshift derived from general relativity [39,40,41,16, with its own caveats, some theories are based on heterodox ideas about gravitation. Broberg [42, for example, pointed out that a comparison between a quantizing scheme inherent in the Schwarzschild metric and observed redshifts from quasars shows that the contracted distances in the gravitational field are quantized in terms of the gravitational radii of the gravitating objects responsible for the field, whilst non-contracted distances are not so quantized. Indeed, physicists have observed quantized states of matter under the influence of gravity: cold neutrons moving in a gravitational field do not move smoothly but jump from one height to another 43 . In Broberg's view, this might be as important for modern quantum physics as the Michelson-Morley Experiment was for the introduction of special relativity. Quantization therefore appears in systems with significant relativistic contraction (gravitational or Lorentz-contraction), such as de Broglie waves or, in the extreme case, photon energy packets at the speed of light. In generalized terms, this would mean that quantum physics has its origin in relativistically contracted fields, whereas the gravitational field can be scaled to particle dimensions, with a constant surface energy density as the invariant parameter in the process. This might, for example, lead to the possibility of a topological transformation from the gravitational force to the strong nuclear force.

Amitabha Ghosh 44] proposed a phenomenological model of dynamic gravitational interaction between two objects that depends not only on their masses and the distance between them, but also on the relative velocity and acceleration between the two. These velocity- and acceleration-dependent interactions are termed "inertial induction". The velocity-dependent force leads to a cosmic drag on all objects moving with respect to the mean rest-frame of a universe treated as infinite and quasi-static. This cosmic drag results in the cosmological redshift of light coming from distant galaxies without any universal expansion. This force model leads to the exact equivalence of gravitational and inertial masses and explains many not well understood observed celestial and astronomical phenomena.

"Self Creation Cosmology" (SCC) [45,46, 47,48, is an adaptation of the Brans Dicke theory in which the conservation requirement is relaxed to allow the scalar field to interact with matter. SCC can be thought of as general 
relativity + Mach's principle + local conservation of energy. In SCC energy is conserved but energy-momentum is not. Particle masses increase with gravitational potential energy and, as a consequence, cosmological redshift, is caused by a secular, exponential increase of particle masses. The universe is static and eternal in its Jordan frame and linearly expanding in its Einstein frame. Furthermore, as the scalar field adapts the cosmological equations, they require the universe to have an overall density of only one third of the critical density and yet be spatially flat. Also in the theory a "time-slip" exists between atomic "clock" time on one hand and gravitational ephemeris and cosmological time on the other, which would result in observed cosmic acceleration.

There is more to be said about gravity and/or interaction with gravitons. In general relativity, dropping the restriction that a global time parameter exists, and assuming instead that the time scale depends on spatial distance, leads to static solutions, which exhibit no singularities, need no unobserved dark energy, and can explain the cosmological redshift without expansion 49. Additional redshift can also be explained using Weyl geometry-gravitation 50 , 51,52,53. Interaction between gravitons and photons $7,54,55,5$ might be responsible for the redshift because it provides an energy loss mechanism that is not subject to the usual problem of making distant galaxy images fuzzy, and because it varies with $(1+z)^{-2}$ which is closer to the observed rate of decrease of surface brightness than any Big Bang model.

Instead of new ideas on gravitation, Roscoe's [56] aim was to shed new light on classical electrodynamics. After abstracting from certain well-known symmetries of classical electrodynamics and showing that these symmetries are alone sufficient to recover the classical theory exactly and unadorned, he found that the classical electromagnetic field is then "irreducibly" associated with a massive vector field. This latter field can only be interpreted as a classical representation of a massive photon. That is, it was demonstrated that existing classical theory is "already" compatible with the notion of a massive photon. He was then able to show that, given a certain natural assumption about how we should calculate the trajectories of this massive photon, the "quantized redshift phenomenology" can be understood in a simple and natural way. Other new ideas rested not on new physics or new reinterpretations of old theories, but on effects predicted at present with standard physics. Also, Mosquera Cuesta et al. [57] propose a non-cosmological redshift that is non-linear in terms of the electrodynamical description of photon propagation through weak background intergalactic magnetic fields. Indeed, Maxwell may have been the first to consider the possibility of non-conservative photon behaviour. His original electromagnetic wave equation contained the energy damping term $\sigma_{0} \mu_{0}(\partial \phi / \partial t)$, where $\sigma_{0}$ and $\mu_{0}$ represented the electrical conductivity and magnetic permeabitity of background space[58](p. 431 of reprinted version of 1954) 59. In 1921, Nernst 60 (p. 40) put forth the idea in an astronomical object by proposing that Olber's paradox might be resolved if photons were assumed to undergo non-conservative energy damping during their journey through intergalactic space. 
Furthermore, just to give further examples from the huge literature on the topic, there are explanations of non-cosmological/non-Doppler redshifts in terms of the local shrinkage of the quantum world 61; or the time variation of the speed of light 62 ; or proposals of a quantum long time energy redshift 63 : the energy is smaller owing to a quantum effect when the photon travels a very long time; chronometric cosmology $[64,65$; the variable mass hypothesis 66,67 3]; time acceleration 68, etc.

All these proposed mechanisms show us that it is quite possible to construct a cosmological scenario with non-expansion redshifts. Nonetheless, all these theories are at present just speculations without direct experimental or observational support.

\subsection{Anomalous redshift in the laboratory and in the solar system}

There are not many experiments that try to measure anomalous (neither Doppler nor gravitational) redshifts in the laboratory. One of these called my attention: Chen et al. 69 reported the observation of line shift increase with plasma electron densities: roughly $\Delta \lambda(\mathrm{nm}) \sim N_{e}\left(10^{18} \mathrm{~cm}^{-3}\right)$ for the HgI line with $\lambda=435.83 \mathrm{~nm}$. The dependence is also influenced by the temperature. This is justified by the authors by the difference in the atomic energy levels for higher electron density, leading to a longer wavelength. The $\mathrm{Hg}$ atomic levels embedded in a density environment are influenced by the free electron density. The electronic fields generated from free electrons compressed inside an atom screen the Coulomb potential of the atomic nucleus. The nuclear forces on the bound electrons are then diminished, while the repulsion of free to bound electrons is enhanced, so that the energy levels outside the nucleus are raised and the intervals of excited energy level $7 s^{3} S$ to $6 p^{3} P_{1}^{0}$ are diminished. Previous studies also pointed out this kind of redshifts [70. My impression is that this emission redshift affects only to some lines and not the spectrum as a whole; in principle one would anticipate a dependence on wavelength. Moreover, it is not shown here that this can explain the absorption of a photon and its emission with redshift (like cosmological redshift), as Ashmore 30] thinks.

The centre-to-limb variation of the wavelengths of solar spectrum was discovered by Halm in 1907. Mérat et al. [71,72 measured in 1974 redshifts in radio of background stars when they are near the Sun, crossing the solar coronal layers, and it is strong near the solar limb. General relativity predicts a deviation of light of $1.75^{\prime \prime}$. However, very near the solar limb, the deviation is $2.03^{\prime \prime}$, whose excess over $1.75^{\prime \prime}$ might be linked with an excess of redshift. Further research was carried out later by several defenders of anomalous redshifts 73 , 29]. However, solar physicists consider the effect to be well explained in terms of standard physics and our knowledge of the convection in the Sun 74. The properties of solar line asymmetries and wavelength shifts can be traced back to the small-scale inhomogeneities in the photosphere 74]: granulation (irregular polygonal shape of 1000-2000 km separated by narrow dark lanes). 
2.5 Observational tests for the expansion of the Universe

Nobody has ever directly observed a galaxy distance being increased with time. The motion of the putative expansion of the Universe is so slow that it is unmeasurable. The variation in the redshift of a galaxy is also very slow and difficult to measure. Sandage 75 proposed in 1962 to measure the expected $\frac{d z}{d t}=(1+z) H_{0}-H(z)(\sim 1 \mathrm{~cm} / \mathrm{s} / \mathrm{yr})$, but it has not hitherto been possible to meassure such a low acceleration. It would be possibly observable with a $40 \mathrm{~m}$ telescope over a period of $\sim 20$ yr using $4000 \mathrm{~h}$ of observing time 76 . So, unfortunately, apart from the actual redshift of the galaxies, there are at present no direct proofs of the expansion. However, there are different indirect tests to verify whether the Universe is expanding or static:

1. Microwave background temperature as a function of redshift.

The Cosmic Microwave Background Radiation (CMBR) temperature can be detected indirectly at high redshift if suitable absorption lines can be found in high redshift objects. Hot Big Bang cosmology predicts that the temperature of the CMBR required to excite these lines is higher than at $z=0$ by a factor $(1+z)$. For a static Universe, there is no specific CMBR model, but one may say that the temperature would be constant and equal to $2.73 \mathrm{~K}$ for all redshifts if there were a local CMBR origin, or possibly $T_{\mathrm{CMBR}}(z)=2.73(1+z) \mathrm{K}$ for a distant $\mathrm{CMBR}$ origin produced at a unique distance and with a redshift due to tired light.

The CMBR temperature measured from the rotational excitation of some molecules as a function of redshift 77,78, has been quite successful in proving expansion: the results of Noterdaeme et al. 78] with the exact expected dependence on $T=T_{0}(1+z)$ are impressive. However, there are other results that disagree with this dependence 79,80 . The discrepancy might be due to a dependence on collisional excitation [77] or bias due to unresolved structure 80.

Nonetheless, there is another piece of evidence of the relationship $T=$ $T_{0}(1+z)$, which comes from the Sunyaev-Zel'dovich effect 81, the imprint of galaxy clusters on the CMBR, and this does not have the problems noted in the previous paragraph. Therefore, if we accept these results, at least one can say with almost total confidence that a local origin of the CMBR in a static Universe is excluded, but the possibility of the origin of the CMBR at very high $z$ within a static Universe is not excluded.

2. Time dilation test.

Clocks observed by us at high redshifts will appear to keep time at a rate $(1+z)$ times slower when there is expansion. By using sources of known constant intrinsic periodicity, we would expect their light curves to be stretched in the time axis by a factor $(1+z)$.

Time dilation tests in Type Ia supernovae (SNIa) look set to become one of the most successful tests in favour of the expansion of the universe 82,83 , but there are still problems in their interpretation. The fact that SNIa light curves are narrower when redder 84 is an inconvenience for a clean test free 
from selection effects. Other selection effects and the possible compatibility of the results with a wider range of cosmological models, including static ones, have also been pointed out [85,86, 87, 88] 37] (Secc. 2) [89] (Secc. 7.8).

Moreover, neither gamma-ray bursts (GRBs) 90] nor QSOs [91] present time dilation, which is puzzling. However, with regard to this last fact, a variation in the quasar flux was found 92 in observations of more than 90 days with an average $\left|d F_{V} / d t\right|$ approximately proportional to $(1+z)^{-1}$, although they used a sample of only 13 QSOs, without K-corrections. Further research is needed for the time dilation of quasars to arrive at firm conclusions.

3. Cosmic chronometers.

Measurements of the Hubble parameter $\dot{z}$ using the variation of the age of elliptical galaxies with redshift as cosmic chronometers 93, 94 might be used to separate the different cosmological models. For the standard cosmological model $-\frac{\dot{z}}{1+z}=H(z)=H_{0} \sqrt{\Omega_{m}(1+z)^{3}+\Omega_{\Lambda}}$, whereas for a static model with tired light $-\frac{\dot{z}}{1+z}=H_{0}$, and for a static model with a linear extrapolation of Hubble's law with redshift $-\frac{\dot{z}}{1+z}=\frac{H_{0}}{1+z}$. The differences in the predictions are huge, and the actual data 93,94 favour the standard model and exclude a static universe. Regrettably, however, the assumptions made for cosmic chronometers are incorrect. It is not true that elliptical galaxies have a single stellar population, they have at least two distinct stellar populations, a young one and an old one, and the use of the Balmer break to measure the age of the galaxy reflects an age difference between both populations, not the maximum stellar age corresponding to the galaxy 95]. This is a serious problem for the use of cosmic chronometers as they are applied nowadays. Therefore, so long we have no accurate methods to determine the age of galaxies, this method is inapplicable.

4. Hubble diagram. It has been known for many decades that an apparent magnitude (taking into account K-corrections) vs. redshift diagram for elliptical galaxies in clusters better fits a static than an expanding Universe 96. This disagreement could, however, be solved by an increase of luminosity at higher redshift due to the evolution of galaxies.

For SNIa 97] or GRBs 98, for which it is supposed there is no evolution, the standard model works, provided that an ad hoc dark energy constant is included and we assume zero evolution and negligible extinction or selection effects (which are not universally accepted; 99, 100, 101]). Nonetheless, a static Universe may also fit those data 102, 103, 104, 105, 106]. By the way, there is anisotropy in the Hubble diagram (values of $H_{0}$ and $\Omega_{\Lambda}$ ) comparing data of SNIa with $z<0.2$ from both Galactic hemispheres [107.

5. The Tolman surface brightness test.

Hubble and Tolman 108 proposed the so-called Tolman test, based on the measurement of surface brightness. A galaxy at redshift $z$ varies in surface brightness proportionally to $(1+z)^{-n}$, with $n=4$ for expansion and $n=1$ for the static case. 
Lubin \& Sandage 109, using the Tolman test up to $z=0.9$, claimed in 2001 to have definitive proof of the expansion of the Universe. However, their claim, rather than being a Tolman test, was that the evolution of galaxies can explain the difference between the results of the Tolman test and their preferred model, which includes expansion. Lerner[110] observed that Lubin \& Sandage used a very involved evolutionary k-correction scheme, with many adjustable assumptions and parameters to correct observed high- $z$ surface brightness. These evolutionary and K-corrections are subject to uncertainty and cannot be used convincingly. Crawford 37 also pointed out that Lubin \& Sandage performed an erroneous analysis to exclude the static solution, mixing Big Bang and tired-light models.

Furthermore, other more recent Tolman tests 110, 111, 112, 37, some of them up to redshifts of $\sim 5$ and with different wavelength filters so that no K-corrections are necessary, favour a static Universe without the need for galaxy evolution, but they cannot exclude the standard scenario if evolution is allowed.

6. Angular size vs. redshift test.

The angular size $(\theta)$ of a galaxy with a given linear size [113] is very different according to whether we assume the standard model with expansion or a static Universe. Tests have been made by several authors 96, 114, 115, 104, and all of them, either in the radio, near infrared or visible, show, over a range of up to redshift 3 , a dependence $\theta \sim z^{-1}$, a static Euclidean effect over all scales. This result cannot be reconciled with the standard cosmological model unless we assume a strong evolution of galactic radii that just coincidentally happens to compensate for the difference [116]: galaxies with the same luminosity should be six times smaller at $z=3.2$ than at $z=0$ [104. It is usually further argued that elliptical and disc galaxies have different evolutionary paths; that is right, but this difference was analysed and found to be compatible with a null one if we take into account the error bars of selection effects [104. Angular-size test for massive disclike galaxies also obtain a good fit for static universes without evolution according to some authors 89. (Secc. 7.4) [117. Neither the hypothesis that galaxies which formed earlier have much higher densities nor their luminosity evolution, merger ratio, or massive outflows due to a quasar feedback mechanism are enough to justify such a strong size evolution [104; also, the velocity dispersion would be much higher than observed[104]. A static Universe is fitted without any ad hoc element. However, we must be cautious with this interpretation, because of the uncertainty in the galaxy size evolution.

There is yet another idea to be found in the literature about such a strong apparent evolution of galaxies: at high redshift, owing to the Tolman factor proportional to $(1+z)^{-4}$, the surface brightness of the galaxies is much lower, and only very compact galaxies are detected (with high surface brightness) for a given stellar mass/luminosity [118. This will give place to a certain "apparent" evolution in the average size of galaxies (smaller 
galaxies being observed at high redshifts), producing a downsizing illusion (dwarf galaxies apparently lift themselves above the sky at recent epochs). So far, there are no standard rods serving as physical objects without evolution. Double radio sources were proposed as standard rods 119 but there is also a strong inverse correlation between their absolute brightness and linear size, and some degree of evolution is not totally excluded 119, 120. Ultra-compact radio sources were claimed in the past to be free of evolutionary effects, but they are now thought to show some signs of evolution as well 104, 121. The huge size evolution necessary to fit an angular size test with an expanding universe is not understood [104] but still depends on our understanding of the galaxies rather than on pure cosmological approaches. The ratio between gas mass fraction in clusters measured through $\mathrm{X}$-ray and gas mass fraction (in the same clusters) measured through the Sunyaev-Zeldovich effect is proportional to the ratio of luminosity distance and angular distance, thus providing a method to derive indirectly the angular size $(\mathrm{z})$, though this method assumes certain mass distributions in the cluster 122 .

7. The galaxy number count magnitude test.

Differential galaxy number counts vs. galaxy K magnitude are fitted by the standard cosmological model 123, but also by the tired light Universe 89 (Sect. 7.7). The same problem arises again: degeneracy between evolution and cosmological geometry.

8. UV surface brightness test.

Lerner 110] proposed a test of the evolution hypothesis that is also useful in the present case. There is a limit on the ultraviolet surface brightness (UV $\mathrm{SB}$ ) of a galaxy because, when the surface density of hot bright stars and thus supernovae increases, large amounts of dust are produced to absorb all the UV except that from a thin layer. Further increase in the surface density of hot bright stars beyond a given point just produces more dust and a thinner surface layer, not an increase in UV SB. Based on this principle, there should be a maximum UV(at-rest) SB independent of redshift. This was analysed at high redshift 110,104 and the result is that the intrinsic UV SB magnitude of some galaxies would be prohibitively lower(i.e. much brighter) than $18.5 \mathrm{mag}_{A B} / \operatorname{arcsec}^{2}$ with the standard model. For a static model, however, it would be within the normally expected range. Lerner 110 also argues why alternative explanations (lower production of dust at high redshift, winds, or other scenarios) are inconsistent. Nonetheless, Lerner's hypothesis of a maximum UV SB might be incorrect, so this should be further explored before reaching definitive conclusions about this test.

9. Alcock-Paczyński test.

Given a distribution of spherically symmetric objects with a radius along the line of sight $s_{\|}=\Delta z \frac{d d_{\text {com }}(z)}{d z}$ and a radius perpendicular to the line of sight $s_{\perp}=\Delta \theta(1+z)^{m} d_{\text {ang }}(z)(m=1$ with expansion, $m=0$ for static $)$, the ratio $y \equiv \frac{\Delta z}{z \Delta \theta} \frac{s_{\perp}}{s_{\|}}$depends on the cosmological comoving distance $\left(d_{\text {com }}(z)\right)$ 
Table 1 Cosmological tests of the expansion.

\begin{tabular}{ccc} 
Test & Expansion & Static \\
\hline \hline$T_{\text {CMBR }}(z)$ & Good fit & $\begin{array}{l}\text { Tired light redshift of a CMBR } \\
\text { coming from very high z. }\end{array}$ \\
\hline Time dilation & $\begin{array}{l}\text { Unexplained absence of time } \\
\text { dilation for QSOs and GRBs. }\end{array}$ & $\begin{array}{l}\text { Selection effects, or ad hoc } \\
\text { modification of the theory or } \\
\text { the zero point calibration, or } \\
\text { evolution of SNIa periods. }\end{array}$ \\
\hline Cosmic chronometer & $\begin{array}{l}\text { Good fit but by chance, } \\
\text { measurements of differential age } \\
\text { are incorrect. }\end{array}$ & $\begin{array}{l}\text { Bad fit but measurements of } \\
\text { differential age are incorrect. }\end{array}$ \\
\hline Hubble diagram & $\begin{array}{l}\text { Good fit but requires the } \\
\text { introduction of dark energy } \\
\text { and/or evolution of galaxies. }\end{array}$ & $\begin{array}{l}\text { Good fit for galaxies. Good } \\
\text { fit for SNIa with some models. }\end{array}$ \\
\hline Tolman (SB) & Requires strong an SB evolution. & Good fit \\
\hline Angular size & $\begin{array}{l}\text { Requires too strong } \\
\text { evolution of angular sizes. }\end{array}$ & Good fit \\
\hline Galaxy counts & $\begin{array}{l}\text { Good fit for galaxies with } \\
\text { evolution. }\end{array}$ & Good fit \\
\hline UV SB limit & Anomalously high UV SB at high z & Within the constraints \\
\hline Alcock-Paczyński & $\begin{array}{l}\text { Bad fit for the } \\
\text { standard model but good fit } \\
\text { with other wCDM models. }\end{array}$ & Good fit for tired light \\
\hline \hline
\end{tabular}

and the angular distance $\left(d_{\text {ang }}(z)\right)$, and is independent of the evolution of galaxies, but it also depends on the redshift distortions produced by the peculiar velocities of gravitational infall 124 .

I have measured $y(z)$ by means of the analysis of the anisotropic correlation function of sources in several surveys 124, using a technique to disentangle the dynamic and geometric distortions, and also took other values available from the literature. From six different cosmological models (concordance $\Lambda$ CDM, Einstein-de Sitter, open-Friedman Cosmology without dark energy, flat quasi-steady state cosmology, a static universe with a linear Hubble law, and a static universe with tired-light redshift), only two of them fitted the data of the Alcock \& Paczyński's test: concordance $\Lambda \mathrm{CDM}$ and static universe with tired-light redshift. The rest were excluded at a $>95 \%$ confidence level. Analyses with further data using Baryonic Acoustic Oscillations (BAO) 125 improve the test and give us a more accurate constraint: $\Lambda \mathrm{CDM}$ with standard values of parameters is excluded at $98 \%$ C.L., whereas static with tired light fits the data, but also an expanding Universe with zero-active mass or ${ }^{\mathrm{w} C D M}$ with $\omega_{\text {dark energy }}=-0.60_{-0.27}^{+0.30}$ and $\Omega_{m}=0.45_{-0.19}^{+0.21}$ fits the data.

Table 1 summarizes the analyses of this subsection. Apparently, there is no winner yet. The first two tests favour expansion, whereas the $4-8$ th tests get a less ad hoc fit with the static solution, although this is insufficient to reject expansion. Most of the cosmological tests are entangled with the evolution of galaxies and/or other effects. Tolman or angular size tests need to 
assume a very strong evolution of galaxy sizes to fit the data with the standard cosmology, whereas the Alcock-Paczynski test is independent of the evolution of galaxies but it does not show any preference for an expanding or static Universe yet.

\subsection{Anomalous redshifts}

Doubt might be cast upon the reality of the expansion as discussed above, but even in the case that it is definitively proven, this does not mean that all galaxies have a cosmological redshift, i.e. that all galaxies have a redshift due to the expansion. There might be some exceptions, which are known as "anomalous redshift" cases 15, 126, 127, 128, 129, 130, 131, 132, 133, 134; that is, a redshift produced by a mechanism different from the expansion of the Universe or the Doppler effect.

There are plenty of statistical analyses $128,135,136,137,138,139,140,141$, $142,143,144$, showing an excess of high redshift sources near low redshift galaxies, positive and very significant cross-correlations between surveys of galaxies and QSOs, an excess of pairs of QSOs with very different redshifts, etc.

There are plenty of individual cases of galaxies with an excess of QSOs with high redshifts near the centre of nearby galaxies, mostly AGN (Active Galactic Nuclei). In some cases, the QSOs are only a few arcseconds away from the centre of galaxies with different redshifts. Examples are NGC 613, 2237+0305, and 3C 343.1. In some cases there are even filaments/bridges/arms apparently connecting objects with different redshift: in NGC 4319+Mrk 205, QSO1327206 , NGC $3067+3 \mathrm{C} 232$ (in the radio). The probability of chance projections of background/foreground objects within a short distance of a galaxy or onto the filament is as low as $10^{-8}$, or even lower. The alignment of sources with different redshifts also suggests that they may have a common origin, and that the direction of alignment is the direction of ejection. This happens with some configurations of QSOs around NGC 4235, NGC 5985, GC 0248+430, etc. Other proofs presented in favour of the QSO/galaxy association with different redshift is that no absorption lines have been found in QSOs corresponding to foreground galaxies (e.g. PKS 0454+036, PHL 1226), or distortions in the morphology of isolated galaxies.

The non-cosmological redshift hypothesis also affects galaxies differently from QSOs. Cases such as NGC 7603, AM 2004-295, AM 2052-221, etc., present statistical anomalies also suggesting that the redshift of some galaxies different from that of QSOs might have non-cosmological causes. Not all supporters of the non-cosmological redshift agree with this idea; for instance, Arp claimed that galaxies might have non-cosmological redshift because they derive from an evolution of ejected QSOs, while Geoffrey Burbidge only defended the non-cosmological redshifts in QSOs.

In addition to the statistical anomalies, QSOs are still nowadays, half a century after their discovery, objects which are not completely understood in other aspects. There are many pending problems, inconsistencies, and caveats 
in the QSO research [145]. The standard paradigm model based on the existence of very massive black holes that are responsible for QSOS' huge luminosities, resulting from to their cosmological redshifts, leaves many facts without explanation. There are several observations that lack a clear explanation; for instance 145, the absence of bright QSOs at low redshifts, a mysterious evolution not properly understood. There are alternative cosmological scenarios, such as the decelaration of time 146, that try to explain the problem without referring to an evolution effect in which the position of QSOs in the Hubble diagram is explained by an improper estimation of luminosity.

Calculations of the probabilities of the anomalous redshift cases being normal background sources with cosmological redshifts and/or with amplifications due to gravitational lensing 134 indicate that some of the examples of apparent associations of QSOs and galaxies with different redshifts may be just fortuitous cases in which background objects are close to the main galaxy although the statistical mean correlations remain to be explained, and some lone objects have a very low probability of being a projection of background objects. Nevertheless, these very low probabilities (down to $10^{-8}$ or even lower, assuming correct calculations) are not extremely low and, if the anomaly is real, one wonders why we do not find very clearly anomalous cases with probabilities as low as $10^{-20}$. Gravitational lensing seems not to be a general solution yet, although it explains some of the anomalies 147, and the requirement that the probabilities be calculated a posteriori is not in general an appropriate answer for avoiding or forgetting the problem.

There are two possibilities: either all cases of associations are lucky coincidences with a higher probability than expected for some still unknown reason, or there are at least some few cases of non-cosmological redshifts. The debate has lasted a very long time, around 50 years, and it is high time to consider making a last-ditch effort to finish with the problem. Every time the problem is mentioned, supporters of the standard view just smile or talk about "a posteriori" calculations, manipulations of data, crackpot ideas, without even reading any paper on the theme. The Arp-Burbidge hypothesis has become a topic in which everybody has an opinion without having read the papers or knowing the details of the problem, because some leading cosmologists have said it is bogus. On the other hand, the main supporters of the hypothesis of non-cosmological redshifts produce analyses of cases in favour of their ideas without too much care, pictures without rigorous statistical calculations in many cases, or with wrong identifications, underestimated probabilities, biases, use of incomplete surveys for statistics, etc., in many other cases 134. There are, however, many papers in which no objections are found in the arguments and they present quite controversial objects, but owing to the bad reputation of the topic, the community simply ignores them. 


\section{Microwave Background Radiation}

The CMBR has been interpreted as the relict radiation of an early stage of the Universe. Its black-body spectrum of $2.7 \mathrm{~K}$ reveals a very small dependence on sky position, of the order of $\Delta T / T \sim 10^{-5}$ excluding the dipole due to the motion of the Earth. Measurements of the anisotropies carried out by several teams of researchers over the last three decades have been claimed to provide information on the structural formation of the Universe, inflation in its early stages, quantum gravity, topological defects if any (strings, etc.), dark matter type and abundance, dark energy or quintessence, the geometry and dynamics of the Universe, the thermal history of the Universe at the recombination epoch, etc. Moreover, the CMBR has also become a source of accurate values for the parameters of this standard model of cosmology, which is usually called "precision Cosmology" 148. The foundations of cosmology are thought to be definitively established, and now a quantitative science is pursued for which the fitting of the power spectrum of the distribution of CMBR anisotropies and a few other data, and which gives us the numerical values of the parameters in the equations governing the entire past, present, and future of our Universe.

Gamow 149] and Alpher \& Herman [150] in the forties and fifties predicted an early stage of the Universe in the Big Bang model that would produce a relic radiation from this fireball that could be observed as a background. However, Gamow and his coworkers were of the opinion that the detection was completely unfeasible 151 . The first published recognition of the relic radiation as a detectable microwave phenomenon appeared in 1964 152. None of the predictions of the background temperature based on the Big Bang, which ranged between $5 \mathrm{~K}$ and $50 \mathrm{~K}$, matched observations 153 , the worst being Gamow's upward-revised estimate of $50 \mathrm{~K}$ made in 1961. In 1965, the year of the discovery, a temperature of $30 \mathrm{~K}$ was calculated for the amount of helium production observed 154 . As the energy is proportional to $T^{4}$, the energy observed is several thousand times less than predicted energy, but it was predicted correctly that it has a perfect blackbody spectrum 155. Although the discovery is attributed to Penzias \& Wilson, who won the Nobel Prize because of it, the radiation was indeed previously discovered, although not interpreted in terms of a cosmological radiation: in 1957, Shmaonov 156 was measuring radio waves coming from space at a wavelength of $3.2 \mathrm{~cm}$ and obtained the conclusion that the absolute effective temperature of radiation background appears to be $4 \pm 3 \mathrm{~K}$, regardless of the direction of the sky. It is also possible that a team of japanese radioastronomers measured this radiation at the beginning of 1950s [151, and indirectly it was found by MacKellar in 1941 as the necessary radiation to excite rotating cyan molecules [151]; Herzberg also mentions in a book in 1950 [157 that there is a strange excitation of molecular spectra, as if a $2.3 \mathrm{~K}$ radiation existed. Anyway, the radiation is there and the question is the origin of this radiation not the discoverer. Is the high energy primordial Universe the only possible scenario? 
3.1 Alternative explanations for the temperature of $2.7 \mathrm{~K}$

There were predictions of CMBR temperature with origin different from the Big Bang [158] by Guillaume, Eddington, Regener, Nernst, McKellar, Herzberg, Finlay-Freundlich, and Max Born. Charles-Edouard Guillaume (Nobel laureate in Physics 1920) predicted in his article entitled "Les Rayons X" ("X-rays", 1896) that the radiation of stars alone would maintain a background temperature of $6.1 \mathrm{~K}[159]$. The expression "the temperature of space" is the title of chapter 13 of "Internal constitution of the stars" 159, 160 by Eddington. He calculated the minimum temperature any body in space would cool to, given that it is immersed in the radiation of distant starlight. With no adjustable parameters, would be $3 \mathrm{~K}$, essentially the same as the observed background (CMBR) temperature. Other early predictions 159, given by Regener 161 in 1933 or Nernst 162 in 1937, gave a temperature of $2.8 \mathrm{~K}$ for a black body which absorbed the energy of the cosmic rays arriving on earth. It was countered that Eddington's argument for the "temperature of space" applies at most to our Galaxy. But Eddington's reasoning applies also to the temperature of intergalactic space, for which a minimum is set by the radiation of galaxy and QSO light. The original calculations half-a-century ago showed this limit probably fell in the range $1-6 \mathrm{~K}[163]$. And that was before QSOs were discovered and before we knew the modern space density of galaxies. In this way, the existence of a microwave background in a tired light scenario 163, 164] was also deduced. But in a tired light model in a static universe the photons suffer a redshift that is proportional to the distance travelled, and in the absence of absorption or emission the photon number density remains constant, we would not see a blackbody background. The universe cannot have an optical depth large enough to preserve a thermal background spectrum in a tired light model 165 because we could not observe radio galaxies at $z \sim 3$ with the necessary optical depth. Therefore, it seems that this solution does not work, at least when the intergalactic medium instead of the shell of the galaxy is responsible for the tired light.

In the fifties, it was pointed out 166,167 that if the observed abundance of He comes from hydrogen fusion in stars, there must have been a phase in the history of the Universe when the radiation density was much higher than the energy density of starlight today. If the average density of the visible matter in the Universe is $\rho \sim 3 \times 10^{-31} \mathrm{~g} / \mathrm{cm}^{3}$ and the observed $\mathrm{He} / \mathrm{H}$ ratio by mass in it is 0.244 (see 44), then the energy which must have been released in producing He is $4.39 \times 10^{-13} \mathrm{erg} / \mathrm{cm}^{3}$. If this energy is thermalized, the black body temperature turns out to be $T=2.76 \mathrm{~K}$, very close to the observed temperature for the CMBR. Hence, there is a likely explanation of the energy of the microwave radiation in terms of straightforward astrophysics involving hydrogen fusion in stars [168. Hoyle et al. 169] also pointed out the suspect coincidence between the microwave background temperature and that of hydrogen in condensation on grains. They postulate that galaxy and star formation proceed very readily when hydrogen is condensed on grains but not when it is gaseous, and the universal microwave background temperature is that associated with 
galaxy formation. The mechanism of thermalization in any of these cases is perhaps the hardest problem.

\subsection{Alternative origin of CMBR}

Dust emission differs substantially from that of a pure blackbody. Moreover, dust grains cannot be the source of the blackbody microwave radiation because there are not enough of them to be opaque, as needed to produce a blackbody spectrum. A solution for the blackbody emission shape might be unusual properties of the dust particles: carbon needles, multiple explosions or big bangs, energy release of massive stars during the formation of galaxies, and so forth. The quasi-steady state model 170 171 argues that there is a distribution of whiskers with size around $1 \mathrm{~mm}$ long and $10^{-6} \mathrm{~cm}$ in diameter with average $\sim 10^{-35} \mathrm{~g} / \mathrm{cm}^{3}$ providing optical depth $\tau \sim 7$ up to redshift $\sim 4$. However, the presence of a huge dust density to make the Universe opaque is forbidden by the observed transparency up to $z \sim 4$ or 5 . A solution might be an infinite universe. An opaque Universe is required only in a finite space, an infinite universe can achieve thermodynamic equilibrium even if transparent out to very large distances. Somewhat similar to the proposal of whiskers is the proposal of the thermalization of "cosmoids", cosmic meteoroids which are also observed in the solar system[172, or the proposal of emission by millimetre black holes 173 .

Another possible explanation is the existence of an aether [174, 175, i.e. a material vacuum, whose emission gives the microwave background emission itself. This aether would be an incompressible fluid according to Lorentz's theory and its existence is in opposition to Einstein's special relativity. Lorentz's 176 invariant laws of mechanics in a flat space-time reproduce the standard observational tests of general relativity provided that rest mass and light speed are not constant, so the final choice between Einstein's and Lorentz's theories cannot yet be regarded as settled according to Clube [174].

The CMBR may be produced by the scattering of photons with electrons in a plasma of temperature 2 million K 29]. Lerner[177, 178, proposes that electrons in intergalactic magnetic fields emit and absorb microwave radiation. There is no relation between the direction in which the radiation was moving when it was absorbed and its direction on re-emission, so the microwaves would be scattered. After a few scatterings, the radiation would be smoothed out. Magnetic fields much stronger than the average field between galaxies would be needed; perhaps the jets emitted from galactic nuclei would provide it. The background radiation would be distorted by this intergalactic absorption against isotropy observations, so the radiation must instead come from the intergalactic medium itself in equilibrium. This prediction agrees with the fact that the number of radio sources increases much more slowly than the number of optical sources with distance, presumably owing to this absorption of radio waves in the intergalactic medium. Observational evidence was also presented that something in the intergalactic medium is absorbing radio and 
microwaves because farther radio sources with a given constant infrared emission are fainter in the radio 179 180. However, there are some sources which are quite bright in radio at intermediate redshift: Cygnus $\mathrm{A}(z=0.056)$ and Abell $2218(z=0.174)$. There is even a constant FIR/radio emission up to $z=1.5$ [181, 182 and sources are observed at $z=4.4$, so unless we have a problem of anomalous redshift in all these cases, which seems unlikely, Lerner's ideas do not work.

Related to electrons, there are other discussions of alternative explanations of CMBR in the literature too. For instance, the radiation of electrons orbiting protons in atoms 183. It is proposed that the atoms of hydrogen emit energy while in equilibrium owing to the orbiting of the electron around protons (which goes against the principles of quantum mechanics), thereby generating the background spectrum of $2.73 \mathrm{~K}$. The atoms emit and absorb this radiation creating a background field with a blackbody shape.

Krishan 184 points out that, in addition to Thomson scattering, the absorption due to the electron-electron, electron-ion and the electron-atom collisions in a partially ionized cosmic plasma would also contribute to the optical depth of the cosmic microwave background (CMB). The absorption depth depends on the plasma temperature and the frequency of the CMB radiation. The absorption effects are prominent at the low frequency part of the CMB spectrum. These effects, when included in the interpretation of the CMB spectrum, may require a revised view of the ionization of the universe.

In "Curvature Cosmology" [185] the CMBR comes from the curvatureredshift process acting on the high-energy electrons and ions in the cosmic plasma. The energy loss which gives way to the spectrum of photons of the CMBR occurs when an electron that has been excited by the passage through curved spacetime interacts with a photon or charged particle and loses its excitation energy.

The origin must be very local in order to preserve the blackbody shape, or be non-redshifted, which seems problematic. Some proposals have even placed the origin of the CMBR within our own Galaxy, in the local bubble within $100 \mathrm{pc}, 186$, positing that the microwave background radiation stems in very large part in re-radiation of thermalized Galactic starlight. But the high level of isotropy, or the Sunyaev-Zel'dovich imprint of clusters of galaxies in the CMBR [187] or any possible correlation of the CMBR with galaxies would be puzzling in terms of such re-radiation 1

Navia et al. 188. find that there is an isotropic distribution of cosmic rays with energy $>6 \times 10^{19} \mathrm{eV}$, so they should come from distances greater than 50 $\mathrm{Mpc}$, and the cosmological interpretation of the CMBR does not allow these cosmic rays to travel distances $\geq 50 \mathrm{Mpc}$ [189,190. However, Abraham et al.191] claim that an isotropic distribution of these cosmic rays is rejected with $>99 \%$ C.L. and that the most probable sources are nearby AGN. Kashti \& Waxman[192 also claim that the distribution of these high energy cosmic

1 This has indeed not been found yet, since the measurements of the cross-correlation of CMBR maps with galaxy surveys are not significant; see Ref. [193] and references therein. 
rays is inconsistent with isotropy at $\sim 98 \% \mathrm{CL}$, and consistent with a source distribution that traces LSS, with some preference to a source distribution that is biased with respect to the galaxy distribution.

In my opinion, at present there is no satisfactory alternative scenario that does not have a problem in explaining the microwave background radiation, so the standard scenario seems to be the best solution, although also with some discussions about its plausibility in some skeptical literature ${ }^{2}$ Nonetheless, one's mind should not be definitively closed against other options. The hot primordial Big Bang is only a hypothesis that should not be definitively considered as a solid theory just because of the Microwave Background Radiation.

\subsection{Microwave Background Radiation anisotropies}

Another fact is the existence of certain anisotropies in CMBR. There are still some authors who doubt this fact, pointing out that there are spurious temperature anisotropies that are comparable with the entire signal [195, 196, 197], or testing the null hypothesis that the Time-Ordered-Data (TOD) were consistent with no anisotropies when hourly calibration parameters were allowed to vary, i.e. that sky maps with no anisotropies outside the galactic band other than the dipole were a better fit to the uncalibrated TOD than those from the official analysis [198. In my opinion, these analyses cannot be correct. The fact that the same anisotropies have been found by many different experiments and by different teams leaves no doubt in my mind that the anisotropies are real.

The first predictions of CMBR anisotropies were wrong. One predicted $\Delta T / T$ to be one part in hundred or thousand [199]; however, this value could not fit the observations, which gave values hundreds of times smaller, so nonbaryonic dark matter was introduced ad hoc to solve the question.

CMBR analyses in the last two decades have concentrated on anisotropies on small angular scales, smaller than the angular resolution of several degrees achieved by COBE-DMR in the '90s. The power spectrum of the anisotropies of the experiments BOOMERANG and MAXIMA-1 showed a first peak at the Legendre multipole $\ell \approx 200,200,201$. corresponding to angular scales of $\approx 1.2^{\circ}$, which was interpreted as a discovery of the previously predicted acoustic peaks in a flat Universe with $\Omega=\Omega_{m}+\Omega_{\Lambda}=1$, the greatest contribution coming from a dark energy component of $\Omega_{\Lambda}=0.7$ (see review in Ref. [202] and references therein for further comments). Indeed, there are two parts in the comparison of theoretical predictions and observations: a successful one, and a half-successful/half-failed one. The totally successful prediction was the qualitative shape of the power spectrum containing several peaks: For instance,

\footnotetext{
2 For instance, the number of (CMBR) photons is much $\left(10^{9}\right.$ times) higher than the number of cosmic baryons, thus indicating that cosmic evolution violates baryon number conservation; a heavy baryon-antibaryon annihilation? Curiously, the CMBR photon density implies that the mean distance of photons is $0.2 \mathrm{~cm}$, which, to the surprise of some, is just about identical to the maximum wavelength of the CMBR black body emission [194].
} 
Peebles \& Yu[203] had predicted a CMBR power spectrum with fluctuations, the acoustic peaks, from the hypothesis of primaeval adiabatic perturbations in an expanding universe. However, the position of the first peak was not at the expected position when first measured: already in the mid-'90s the position of the first peak was determined to be $\ell \approx 200$ with other experiments to measure small angular resolution anisotropies before BOOMERANG or MAXIMA-1 but with smaller sky coverage (Ref. 204 and references therein). De Bernardis et al.200 and Hanany et al.201 just added a refinement in the measurement of the position of the first peak but they were not its discoverers. White et al. in 1996204 realized that the preferred standard model of the time (an open Universe with $\Omega=\Omega_{m} \approx 0.2$ and without dark energy) did not fit the observations, so that they needed a larger $\Omega$. This was one of the elements, together with SNIa observations, and the age problem of the Universe, that would encourage cosmologists to include the new ad hoc element: dark energy. Between 1997 and 2000 this change of mentality in standard cosmology was produced, and then, in 2000, with the results of the new BOOMERANG and MAXIMA-1 experiments, cosmologists were proud to announce that new observations were giving exactly the results they expected. In any case, just paying attention to the first analyzes of the first peak in the mid-'90s, I would attribute a half-success to the prediction because, even though they failed to fit the observations with the preferred standard model of a curved open Universe at that time, the idea of a flat Universe was also presented as a possibility in the '90s, and indeed a possibility preferred by inflationary paradigms. The position of the other peaks would also serve to constrain the cosmological models. The acoustic peaks on angular scales of $1 \mathrm{deg}$ and $0.3 \mathrm{deg}$ were predicted with the second peak nearly as high as the first one in $\ell(\ell+1) C_{\ell} /(2 \pi)$ [205, 206]. Later, as data became available, its amplitude was reduced and the positions of the other peaks and their relative heights were also constrained from the model with a higher agreement with the data (e.g. Ref. [207]).

More recently, the analysis of CMBR polarization anisotropies (e.g. Ref. 209] ) has also provided strong support for the standard cosmology. This indicates the way in which the CMBR is polarized owing to the scattering of free electrons. Photon diffusion into regions of different temperatures are possible only when the plasma becomes sufficiently optically thin; these diffused photons could then scatter only while there are still free electrons left. Since photons could not diffuse too far, polarization cannot vary much over very large angular scales.

Given this history of CMBR analyses, it is difficult to accept that our observations reproduce "predictions". Nonetheless, whichever comes first, theory or observation, the fact remains that we have now a cosmological model that is able to fit the CMBR power spectrum $C_{\ell}$ quite accurately. It is usually thought that this may not happen by chance, given the apparent complexity of the power spectrum shape, whereas the six ${ }^{3}$ parameter cosmological model

\footnotetext{
3 Plus many other parameters which introduce second-order changes. And, even so, there is a degeneracy in the solutions with different values of $H_{0}$ and $\Omega_{\Lambda}$ : CMBR data, and the large scale structure of galaxies could be reproduced without explicitly requesting the exis-
} 
is relatively simple 209,210, but this is not correct, as I will explain in the following paragraphs.

Some critics of the standard model 211 claim that there is little value in the fact that the standard cosmological model might fit some observational cosmological data if the number of free parameters in the model were comparable to the number of independent parameters characterizing the observations. For instance, Ptolemaic geocentric astronomy may fit the observations of the orbits of the planets but with too high a number of free parameters in the theory. The principle of Occam's razor tells us that a theory is better when the number of free parameters is low. Occam's razor can indeed be understood with a Bayesian analysis, in which it is tested how probable a model is with a given number of free parameters with respect to other models with a higher number of free parameters 212,213. Otherwise, if a theory has a high number of free parameters, it loses credibility because it is always possible to create a "false" model to fit some data when the number of free parameters is comparable to the number of degrees of freedom in the data. Philosophers of science, when talking about cosmology, associate this approach with "instrumentalism" (e.g., Ref. [214]). And saying that the power spectrum contains hundreds of independent parameters for a given resolution is not correct, because the different values of $C_{\ell}$ for each $\ell$ are not independent in the same sense that hundreds of observations of the position and velocity of a planet do not indicate hundreds of independent parameters. Indeed, the information on the orbit of planet is reduced to only six Keplerian parameters.

For the reasons just given, understanding how much information is in the power spectrum is important for key questions in the discussion of the fundamentals of cosmology. There are indeed two main points which should be clarified:

1. Are the oscillations something atypical in a power spectrum? That is, should we consider the fact the power spectrum contains oscillations a successful prediction of the standard cosmological model that cannot be produced by any other means?

2. How much information is contained in the power spectrum? That is, how many free parameters in a function are necessary to fit the CMBR power spectrum? Should we consider the fitting of the power spectrum with a model of six free parameters as a validation of the standard cosmological model?

For the answer to the first question, we must bear in mind that the presence of peaks in the power spectrum is a rather normal characteristic expected from any fluid with clouds of overdensities that emit/absorb radiation or interact

tence of dark energy 208 i.e. with $\Lambda=0$. This degeneracy is broken by adding cosmological information from other sources, for instance, from SNIa data. In order to fit the temperaturepolarization cross power spectrum and the polarization-polarization power spectrum 209, one would need an extra parameter (optical depth), so a total of at least seven free parameters are necessary. Roughly speaking, the relationship between temperature-temperature and the polarization-temperature, or polarization-polarization power spectra is expected since they are different ways of seeing the same light with different filters. 
gravitationally with the photons, and with a finite range of sizes and distances for those clouds. Apart from the standard cosmological model, other scenarios may also follow these conditions. The interpretation of "acoustic" peaks is just a particular case; peaks in the power spectrum may be generated in scenarios that have nothing to do with oscillations due to gravitational compression 215 , 216 .

For the second question, we must note that a simple polynomial function with no physical interpretation with six free parameters is able more or less to reproduce the two-point correlation function (the Fourier transform of the power spectrum), giving very good fit for at least the first two peaks of the power spectrum 216. The fact the standard model is able to fit the CMBR anisotropies with a model with six free parameters is astonishing but it would be much more surprising if it could fit it without free parameters or with only one or two free parameters fitted to the power spectrum. Certainly, the same parameters which are used to fit the CMBR power spectrum are able to fit other cosmological data, but more than six parameters are necessary, as well as additional information such as initial conditions, conditions of stellar formation, galaxy formation, how dark matter is distributed in galaxies, etc. A global analysis of the cosmological models would require the examination of all of the available independent sources of cosmological data (nucleosynthesis, supernovae, gravitational lensing, etc.) and to check whether they are comparable to the number of free parameters in the model.

In cosmologies different from the standard model, there were also attempts to fit the CMBR power spectrum with models with a similar number of free parameters. Narlikar et al. 217,218 fitted the $C_{\ell}$ with five-six parameters apart from the amplitude within a model that has nothing to do with the origin of the CMBR in the standard cosmology. Power spectrum peaks at $\ell \approx 6$ to 10 , 180 to 220 , and 600 to 900 are shown to be respectively related in their QuasiSteady State cosmology to curvature effects at the last minimum of the scale factor, clusters, and groups of galaxies. Previously, it had been calculated that clusters of cold clouds in the halo produce anisotropies, which peak at $l \approx 50$, has a power proportional to $1 / \sin ^{2} b$ and can represent around $5 \%$ of the total anisotropies at $b=30^{\circ}$ [219]. For a MOND (Modified Newtonian Dynamics) cosmology without cold dark matter, it is also possible with few parameters to fit the power spectrum 220,221. It is not clear, however, that these alternative scenarios can explain the phase coherence needed to account for the clear peak/trough structure observed in CMBR anisotropies, as predicted by an inflationary scenario in the standard model[222].

\subsection{Non-gaussianity}

Another relevant feature of CMBR anisotropies is the gaussianity of its fluctuations. Theories involving inflation generally predict a pattern of Gaussian noise, whereas theories based on symmetry breaking and the generation of defects have more distinctive signatures 223 . However, this should not be a 
major problem for alternative cosmologies since many different origins of the fluctuations are expected to be Gaussian. And even if there may be phenomena that generate deviation from normal Gaussian fluctuations, if many of them intervene, the result will be Gaussian anyway.

It is not totally clear yet whether the CMBR fluctuations are exactly Gaussian: a non-Gaussian distribution has been claimed by several authors (e.g., Ref. [224], §2.3 and references therein; Refs. [225, 226, 2227,228, 229, 230,231]); however, other analyses 232,233 . claim that only a few regions have such nonGaussian anisotropies owing to contamination, e.g. the Corona Borealis supercluster region, and most of the regions in the sky are Gaussian. The nongaussianity may be associated with cold spots of unsubtracted foregrounds 234 235; even the lowest spherical harmonic modes, which should be the cleanest, in the map are significantly contaminated with foreground radiation 236].

This might mean that analyses claiming non-gaussianity are wrong, or that the gaussianity is not significant enough, or that the inflation model is incorrect, or that some contamination is present in the maps, which are supposed to be clear of any contamination. Among all these possibilities, maybe more than one applies, and I suspect that one of these is the incorrect subtraction of the Galactic contamination, although other factors may also be important too.

3.5 Some doubts on the validity of the foreground Galactic contribution subtraction from microwave anisotropies

Many authors have studied the different components of the Galactic microwave foreground radiation, but in my view these efforts are still insufficient to separate the components appropriately. The errors in the foreground emission subtraction are small, but they are not negligible. At least, some doubts on the validity of the foreground Galactic subtraction from microwave anisotropies can be expressed 224$]$.

The question of the Galactic foregrounds has been present for a long time now. However, an analysis of the papers analysing the problem reveals that it has sometimes been underestimated, and that the problem of Galaxy subtraction has become more complex in recent years. For instance, two decades ago it was thought 237, 238 that Tenerife data at $15 \mathrm{GHz}$ were likely to be dominated by cosmological fluctuations, and that the $33 \mathrm{GHz}$ data were scarcely affected by the Galaxy ( $\sim 4 \mu \mathrm{K}$ in scales of $5-15$ degrees). Later analyses (WMAP, 239 (Fig. 10)), however, have shown that for comparable angular scales the Galaxy is dominant in the anisotropies at $22.8 \mathrm{GHz}$ (and the Galactic contamination at $15 \mathrm{GHz}$ is not much smaller than at $22.8 \mathrm{GHz}$ ), and at 33 $\mathrm{GHz}$ the Galactic anisotropies are of the same order as those from the CMBR. Also, at small scales, less than a degree, the foreground Galactic emission dominates 240. An explanation for this is that it is mainly due to the existence of a kind of emission unknown in the early ' 90 s, which is correlated with the dust that was not discovered previously; the synchrotron and or free-free emis- 
sion might also have been underestimated. Positive correlations between the microwave anisotropies, including the region around $15 \mathrm{GHz}$, and far-infrared maps, which trace Galactic dust, were found 240,241,242,243. In the Helix region the emission at $31 \mathrm{GHz}$ and $100 \mu \mathrm{m}$ are well correlated [244]; the 100 $\mu \mathrm{m}$-correlated radio emission, presumably due to dust, accounts for at least $20 \%$ of the $31 \mathrm{GHz}$ emission in the Helix (so the total dust emission is higher than $20 \%$ because there is a non-correlated component too). The anomalous emission at around $23 \mathrm{GHz}$ of the Perseus molecular cloud (temperature $\sim 1$ $\mathrm{mK}) 245$ is an order of magnitude larger than the emission expected from synchrotron + free-free + thermal dust. There is also some correlation of 10 , $15 \mathrm{GHz}$ maps with $H_{\alpha}$ maps, but very low, so the free-free emission is detected at levels far lower than the dust correlation [246, 247]. The most likely current explanation for this emission correlated with dust around $15-50 \mathrm{GHz}$ is spinning dust grain emission 248] and/or magnetic dipole emission from ferromagnetic grains 249. More recently, a new foreground was discovered for low frequencies: "microwave haze" emission around the Galactic center 250. Whatever it is, it is now clear that the $15-40 \mathrm{GHz}$ range is dominated by the Galaxy. A question might arise as to whether the contamination in the remaining frequencies $(40-200 \mathrm{GHz})$ is being correctly accounted for. Typical calculations for dust contamination claim that it should not be predominant, and that its contribution can be subtracted accurately, but how sure can we be of this statement? How accurate is the subtraction of the dust foreground signal?

A first difficulty in the subtraction of the dust component is to know exactly how much emission there is in each line of sight. First approximations came with extrapolations from the IRAS far-infrared and DIRBE data (with the zodiacal light subtracted, as well the cosmic infrared background) used to model the dust thermal emission in microwaves. Templates were taken from these infrared maps and extrapolated in amplitude by a common factor for all pixels. The problem is that, as said by Finkbeiner et al. 251], "a template approach is often carelessly used to compare observations with expected contaminants, with the correlation amplitude indicating the level of contamination. (...) These templates ignore well-measured variation in dust temperature and variations in dust/gas ratio." The growing contrast of colder clouds in the background of the diffuse interstellar medium will produce much higher microwave anisotropies than the product of the template extrapolation 252 . Neither is it a good strategy to subtract a scaled IRAS template to remove the spinning dust in multifrequency data since it produces large residual differences 240. There is a presence of residual foreground emission not traced by the templates 253. A better approximation to this dust emission in the microwave region came with the adoption of an extrapolation with colour corrections in each pixel. This method assigns a different temperature to each pixel. This colour correction, together with a $\nu^{2}$ emission emissivity 254, gave a much tighter agreement with FIRAS data. However, it was still inconsistent with the FIRAS data below $800 \mathrm{GHz}$ in amplitude 251: a 14\% error in the mean amplitude at 500 GHz. Indeed, no power-law emissivity function fits the FIRAS data in the 200- 
$2100 \mathrm{GHz}$ region 251. Furthermore, laboratory measurements suggest that the universality of $\nu^{2}$ emissivity is an oversimplification, with different species of grains having different emissivity laws 251. A better approximation is an extrapolation with two components $\left(\nu^{1.7},\langle T\rangle=9.5 \mathrm{~K}\right.$ and $\left.\nu^{2.7},\langle T\rangle=16 \mathrm{~K}\right)$; each pixel has an assignation of two temperatures [251] with correction factors that are a function of these temperatures. This still fails in the predictions of FIRAS from the IRAS-DIRBE extrapolation by $15 \%$ in zones dominated by atomic gas 251.

Finkbeiner et al.'s 251 approach in 1999, although much better than a direct extrapolation of the template, was insufficient. The problem is difficult to solve because it is an extrapolation by a factor $\sim 15-60$ in frequency (from $1250 \mathrm{GHz}[240 \mu \mathrm{m}]$ or $3000 \mathrm{GHz}[100 \mu \mathrm{m}])$ to $50-90 \mathrm{GHz})$. This is equivalent, for instance, to the attempt to derive a map of stellar emission in $12 \mu \mathrm{m}$ as an extrapolation of the emission in the optical B-filter. Frankly, when I see the IRAS $-12 \mu \mathrm{m}$ map of point sources and the Palomar plates in blue filters, I observe huge differences, and I do not know how we can extrapolate the second map to obtain the first one. Each star has a different colour, and stars which are very bright in blue may be very faint at mid-infrared and vice verse. The same thing happens with the diffuse + cloud emission: there are hot regions, cold regions, different kinds of emitters (molecular gas, atomic gas) and we have to integrate all this into each line of sight. The assumption that with only two temperatures we can extrapolate the average flux, and that a colour term can correct the pixel-to-pixel differences is comparable to the assumption that a model with only two kinds of stars and the knowledge of $(B-V)$ for each star we can extrapolate the star counts from optical to mid-infrared for the whole Galaxy. It is also very common 257,243 to calculate the Galactic dust contribution in some microwave data by just making a crosscorrelation between these data and some far-infrared map of the sky. This is simply wrong and not even valid for ascertaining the order of magnitude of such contamination. Nowadays, things are carried out with much better data and with more frequencies (for instance, with the PLANCK data[255]), but the philosophy of extrapolation and correlation is still similar, and the maps which are supposed to be free of contamination still present signs of dust contamination 256.

Therefore, any method of foreground subtraction which uses templates will have serious credibility problems with regard to the goodness of the subtraction. This applies not only to those methods that use templates directly with coupling coefficients derived from cross-correlation but also to MEM (maximum entropy method; 239]), which uses in the initial stage templates for the dominant foreground components and also establishes some a priori conditions of their spectral behaviour. Moreover, any calculation of the limits of such contamination based on cross-correlations will not be totally accurate.

The presence of different dust-emitting components causes the spectral indices of the foregrounds to vary with position. Spectral index changes from -5.1 to -2.1 , depending on the region have been measured 258, and also changes with wavelength; they point out the existence of dust at different 
temperatures, in particular of a cold, extended component. A catalogue of Galactic cold clumps derived with PLANCK gives spectral index between 1.4 and 1.8 [259]. Even small spectral index variations as small as $\Delta \alpha \sim 0.1$ can have a substantial impact on how channels should be combined and on the attainable accuracy 260, so the errors of the subtraction assuming certain power spectrum for the dust are serious.

Another technique that does not use a templates is ILC (internal linear combination) 239. It assumes nothing about the particular frequency dependencies or morphologies of the foregrounds and tries to minimize the variance in different regions of the sky with the combination of the available frequencies. There is a degeneracy of solutions, an infinite number of maps can be generated, and there is no way to test whether the maximum likelihood solution is the correct one 261]. Those that have applied this method 239] warn against its use for cosmological analysis; it is not effective in removing all residual foregrounds 262 . The ILC method performs quite badly, especially for dust 262, in part because of the variability of the spectral indices. Therefore, ILC maps are not clean enough to allow cosmological conclusions to be arrived at [262]: '[The] ILC map, which by eye looks almost free of foreground residuals, has been extensively used for scientific purposes-despite the fact that there are strong (and difficult to quantify) residual foregrounds present in the map. (...) the ILC map is indeed highly contaminated by residual foregrounds, and in particular, that the low- $\ell$ components, which have received the most attention so far, are highly unstable under the ILC cleaning operation' (Eriksen et al.[263]). ILC provides satisfactory results only under rather restrictive conditions 264 .

The WI-FIT method ("Wavelet based hIgh resolution Fitting of Internal Templates" 265]) does not require a priori templates, but takes the information about the foregrounds by taking differences of temperature maps at different frequencies. However, for the application in presently available maps, it requires the assumption that the spectral indices are constant in space, which, as said, is a very inaccurate approximation. Their assumption that the Galactic emission in each pixel is proportional to the difference in temperature maps $\left(\left(T_{i}^{\nu}-T_{i}^{\nu^{\prime}}\right) \propto T_{i}^{\nu}\right)$ is in general incorrect because $T_{i}^{\nu^{\prime}}$ is not proportional to $T_{i}^{\nu}$, the temperature in each pixel being the superposition of many different emissions with different temperatures. Again, we have here the same problem as with the use of templates: the assumption that there is only one temperature along each line of sight, and that the intensity of this emission is describable with a simple average fixed power law multiplying a black body emission with an average temperature. It has been claimed 265] that their method is good because they obtain similar results to other authors with different methods [239], but this may be due to their similar assumptions.

Foreground contamination residuals are found even for the best available supposedly clean CMB maps. Some correlation is found between $\Delta \ell=4 n$ and $n=1,2$ spherical harmonic multipole domain, which is caused by a symmetric signal in the Galactic coordinate system 266. The alignment of low- $\ell$ multipoles appears to be rather robust to Galactic cut and different foreground 
contaminations 267]. The statistical anisotropy in different circles of the sky is also found 268, pointing that foreground contamination residuals are found even for the best available supposed clean CMB maps. Isotropy of the ILC maps is ruled out to confidence levels of better than $99.9 \%$ 269. The crosscorrelation between CMBR data and $\gamma$-ray data has been studied 270] and it was concluded that an unknown source of radiation, most probably of galactic origin, is implied by their analysis. This unknown radiation of galactic origin might take place in the surface of Galactic HI structures moving through interstellar space and/or interacting with one another 271. The spatial association on scales of 1-2 degrees between interstellar neutral hydrogen[271, integrated in maps over ranges of $10 \mathrm{~km} / \mathrm{s}$ and CMBR maps cleaned of foreground contamination through the ILC methods, is especially significant for the present discussion too. Several extended areas of excess emission at high galactic latitudes $\left(b>30^{\circ}\right)$ are present in both maps. These structures are thought to have typical distances from the Sun of order $100 \mathrm{pc} 271$.

Hence, considering only the Galactic dust component, the methods used to remove it (templates, cross-correlations, assumption of a Galactic power spectrum, MEM, ILC, etc.) are all inaccurate and one should not expect to produce maps clean of Galactic dust contamination by applying them. The analysis of galactic latitude dependence of these anisotropies and the fact that the power spectrum is almost independent of the frequency over the range 50$250 \mathrm{GHz}$ can be considered at least as a proof that the Galactic dust emission is lower than $10 \%$ on the $\sim 1$ degree scale [or double of this value considered to $2 \sigma]$, possibly higher for lower $\ell$ multipoles 224. This uncertainty in Galactic contamination may produce important systematic errors in some cosmological parameters. In any case, one thing is clear: the present error bars calculated for cosmological parameters are very significantly underestimated, and the range of possible values is not as small as indicated by the claims of "precision cosmology".

Galactic contamination is also important for polarized CMBR light. Synchrotron radiation of the Galaxy is strongly polarized; it is observed in radio (408 MHz), and it has loops with an excess of $\sim 20 \mu \mathrm{K}$, probably due to shells of very old supernova remnants 272, which are not being taken into account in the subtraction of foregrounds for polarized CMBR light 273. Thermal dust also leaves an imprint on polarized light. As a matter of fact, the recent claim of the discovery of proofs of gravitational waves due to inflation announced with great ballyhoo, based on the BICEP2 detection of a $\sim 0.3 \mu \mathrm{K}$ B-mode signal, was later shown to be a fake result owing to defective analysis of Galactic contamination. The measurements were done in a region of a loop with important radio emission and with some contamination of dust too 273, 274. The anomalous radiation has the spectrum of magnetic dipole radiation. 
3.6 Other contaminants and anomalies

Apart from the cosmological and the Galactic signal, there may be other contaminants: either from closer sources (in the solar system or the solar neighbourhood), or extragalactic sources much closer than $z=1000-1500$ (as it is supposed for the cosmological origin). The two lowest cosmologically interesting multipoles, $\ell=2$ and 3 , are not statistically isotropic 275, 276]. The planes of the quadrupole and the octopole are unexpectedly aligned [275, 276, 277, 278 at $99.6 \%$ C.L. 276 . This alignment can be regarded as a generalization of the alignment of the $\ell=2$ and 3 modes - the so-called 'Axis of Evil'. Indeed, the combined quadrupole plus octopole is surprisingly aligned with the geometry and direction of motion of the solar system: the plane they define is perpendicular to the ecliptic plane and to the plane defined by the dipole direction, and the ecliptic plane carefully separates stronger from weaker extrema, running within a couple of degrees of the null-contour between a maximum and a minimum over more than $120^{\circ}$ of the sky.

Moreover, the angular two-point correlation function at scales $>60$ degrees in the regions outside the Galactic cut is approximately zero in all wavebands and is discrepant with the best fit $\Lambda$ CDM inflationary model: $99.97 \%$ C.L. for the discrepancy 276, 279, which points towards a violation of statistical isotropy [280]. The lack of quadrupole CMB signal is, according to some authors 281, 278, a serious challenge to the standard model. There is abnormally high (low) powers in the $\ell=6,12-17$ modes [277]; the probabilities for having the anomalous amplitudes of the $\ell=5,6,17$ modes are about $0.1 \%$, $1 \%$ and $1 \%$ respectively according to the Gaussian conjecture. The ratio of the large-scale fluctuation amplitudes in the southern ecliptic hemisphere is high at the level 98-99\% 282, with an absence of large-scale power in the vicinity of the north ecliptic pole. This asymmetry is stable with respect to frequency and sky coverage. Also, the first peak in the anisotropies does not have a blackbody shape and shows a clear hemispherical anisotropy 283 .

However, the WMAP (Wilkinson Microwave Anisotropy Probe) team [284] did not find a significant detection of hemispherical or dipole power asymmetry across the sky. They also claim that the cold spots in the map are statistically consistent with random CMB fluctuations (a claim that is contradicted by later analyses of Planck data 285]); that the amplitude of the quadrupole is well within the expected $95 \%$ C.L.; that there is no anomaly in the lack of large angular CMBR power. The WMAP team 284 admit, however, that there is a remarkably unexpected alignment of the quadrupole and octopole.

A solution to explain some of these anomalies is the presence of a certain amount of contamination in relation with the solar system or its neighbourhood, although part of the effect may be due to non-uniform sky coverage 286. too. If a hypothetic foreground produced by a cold spot in the Local Supercluster is subtracted from the CMBR data, the amplitude of the quadrupole is substantially increased, and the statistically improbable alignment of the quadrupole with the octopole is substantially weakened, but this does not explain the coincidence of the alignment with the ecliptic 287]. 
Anomalies of CMBR data could also be explained by synchrotron radiation emitted in the heliosheath $288,289,290$. Radiative and plasma/Magnetohydrodynamic processes within the heliosheath could affect the propagation of an otherwise isotropic CMBR background through it. The geometric distortion of the termination shock might be a possible source for the quadrupole. Freefree thermal radiation which involves pick up ions at the termination shock region can explain both the blackbody quadrupole and the non-blackbody component to the quadrupole. There is also a prediction for the octopole.

If there were no such contamination and the tension of the low quadrupole, the low octopole, and the alignment of the quadrupole and octopole were real, the tension could be alleviated with a different cosmological model; for instance, $R_{h}=c t$ cosmology [291].

Another consideration that could point to the importance of the noncosmological extragalactic contamination over photons of cosmological origin is that the simulations of the gravitational lensing of the microwave background by galaxy clusters at $z<1$ under any plausible Big Bang model variation produces far more dispersion in the angular size of the primary acoustic peaks than the observations allow 292]. When all the effects are taken together, it is difficult to understand how CMBR data could reveal no evidence whatsoever of lensing by groups and clusters. Cool spots in the microwave background are too uniform in size to have travelled from $z=1000-1500$ to us. There should be a spread of sizes around the average, with some of these cool spots noticeably larger and others noticeably smaller. But this dispersion of sizes is not seen in the data. Too many cool spots have the same size. Moreover, the observed Sunyaev-Zel'dovich effect caused by the clusters only accounts for about $1 / 4$ of the expected decrement 293; although the level of SunyaevZel'dovich effect is observed as in the predictions in radio 294. Effects such as a central cooling flow in clusters, the abundance of hot cluster gas, large scale radial decline in the temperature, uncertainties in the $\beta$-model and the role played by cluster radio sources are too weak to change the estimation 293]. Under Big Bang premises, this implies that the cosmological parameters (including the Hubble constant, the amount of dark matter, etc.) used to predict the original, pre-lensed sizes of the cool and hot spots in the microwave background might be wrong, or that some of these cool spot structures are caused by nearby physical processes and are not really remnants of the creation of the Universe; or perhaps there is some other, unknown factor damping the effects of dispersion and focusing. It was speculated that the large-scale curvature of space may not entirely be an initial value problem related to inflation. The absence of gravitational lensing of the CMBR points to the possibility that even effects on light caused by wrinkles in the space of the late (nearby) Universe have been compensated for, beyond some distance scale, by a mechanism that maintains a flat geometry over such scales. Or high energy electrons may synchrotron radiate in the intracluster magnetic field of strength $\mathrm{B}<\sim 1 \mu \mathrm{G}$ to produce cluster microwave emissions that account for the missing SunyaevZel'dovich effect flux 295]. However, one is also tempted to interpret this in 
terms of the importance of extragalactic non-cosmological contribution in the microwave anisotropies.

Summing up, even if we accepted a cosmological origin for most of the CMBR radiation, putatively clean maps of CMBR anisotropies are most probably not totally free from from solar system, Galactic, or extragalactic contamination, and an accurate way of correcting for all these contributions has still to be devised since we do not have accurate information on the microwave emission of any of these contributions.

\section{Nucleosynthesis and reionization}

\subsection{Light element abundances and baryon fraction}

In the standard model, it is claimed that ${ }^{4} \mathrm{He}$ and other light elements (Deuterium, ${ }^{3} \mathrm{He},{ }^{9} \mathrm{Be},{ }^{7} \mathrm{Li}$ ) were created in the primordial Universe, and the existence of these elements is used as a proof for the necessity of a hot Universe in the past. However, there are alternatives. The alternatives may have some caveats to explain all observations accurately, but neither is Big Bang theory free of problems.

Helium could be created with several explosions such as those in the (quasi)steady state theory, or could be synthesized in massive objects evolving in the nuclear regions of galaxies 296. The existence of massive stars in the first moments of the formation of galaxies, which in few hundred million years would produce the $24 \%$ helium now observed, and this would be distributed through the interstellar space by supernova explosions. Indeed, Burbidge \& Hoyle 297. have argued that a case can be made for making all the light nuclei in stars. Population III stars are believed to contribute to the observed near-infrared background and heavy element pollution of the intergalactic medium, and it could contribute to the primordial He abundance 298. Some theoreticians object that in such a case there should be more oxygen and carbon than observed; this could be solved if the least massive stars had not exploded but blew off their outer layers (pure helium) 28 (ch. 6). Certain rare light isotopes cannot have been produced in this way, but the cosmic rays generated by early stars, colliding with the background plasma, would generate them. Therefore, there are alternatives to the Big Bang to produce any of these elements, although, of course, the standard model is the proposal that is the most complete in detail up to now.

It is said that ${ }^{4} \mathrm{He}$ and ${ }^{7} \mathrm{Li}$ abundances are all consistent with those expected minutes after the Big Bang, provided that the present universe has a baryon density in the range $0.018<\Omega_{b} h^{2}<0.022$ [299], but this statement is not totally free of controversy. The best known abundance is that for ${ }^{4} \mathrm{He}$, around $Y_{P}=0.24$, when the metallicity tends to zero. However, there are claims of some problems with the model: measurements of the abundance of helium of $Y_{P}=0.2565 \pm 0.0010$ (stat.) \pm 0.0050 (syst.), higher at the $2-\sigma$ level than the prediction of standard Big Bang nucleosynthesis 300. Furthermore, 
galaxies of poor metal content, implying minimal stellar contamination, show the mass fraction of ${ }^{4} \mathrm{He}$ as low as 0.21 301. Even allowing for error bars, this is far too low to match the Big Bang predictions. Only an ad hoc explanation of inhomogeneities in the primordial set-up conceals the big bang nucleosynthesis with this result. There are also stars in our own Galaxy that have a low helium abundance in their atmosphere (the subdwarf B stars), but it was shown 302 that these also revealed other peculiarities, such as phosphorus and similar elements, which were associated with the chemically peculiar stars of population I. It was believed that these objects do not show a normal helium abundance and were not taken into account. Also, anomalously low helium content (lower than primordial helium) is required to fit the luminosities and temperatures of the metal-poor $\mathrm{K}$ dwarfs 303 .

According to some authors, the Big Bang also fails in its predictions of the abundance of lithium. The $\mathrm{Li}^{7}$ abundance is much lower than the prediction 304 , 305, 306. The Li problem remains and is indeed exacerbated; the discrepancy is a factor $4.2 \sigma$ (from globular cluster stars) to $5.3 \sigma$ (from halo field stars) 307. This observation of the "Spite Plateau" (the name given to the baseline in the abundance of lithium found in old stars orbiting the galactic halo) is relevant since it is believed that such stars were formed early in the universe out of material that had not been significantly modified by other processes. It was suggested that this discrepancy could be caused by a modification of surface lithium abundances during the stars' lifetimes [308]: the lithium is destroyed in old, metal-poor halo stars. This idea has some support in the fact the lithium in the interstellar medium of the Magellanic Clouds, where the destruction process cannot happen, is in agreement with Big Bang nucleosynthesis predictions (4 times larger than in metal-poor halo stars) 309 .

The evolution of deuterium and the mechanisms of possible deuterium production from the Big Bang are still not properly understood 310,311. For beryllium, excessive abundances are observed in the stars 312, but this is justified with the argument that they have no null metallicity and the abundance should be lower in the primordial Universe. It is also argued that our Galaxy produces beryllium, the accretion of matter 312], or that primordial nucleosynthesis should be a posteriori substituted by inhomogeneous nucleosynthesis 313 .

Still, as we have seen, some open questions are discussed about nucleosynthesis, and whether this can be substituted by alternative models remains to be seen. The question is not as simple as the Big Bang making a unique prediction and the observations confirming it; there is a lot of 'cooking' behind these numbers, a lot of astrophysical processes on element production and evolution that are proposed ad hoc in order to fit some observations that do not fit the a priori predictions, and even so there remain problems to explain everything. There are also some cosmological parameters to play with: the neutron decay time, the number of neutrino species, the ratio between baryons and photons and others; although perhaps the first two are more or less known. Curiously, a much better fit to primordial nucleosynthesis to observations is given when 
the number of neutrino species is two instead of three, as predicted by the standard model for particles 314 .

Perhaps the most important aspect to criticize is the methodology itself for testing the primordial nucleosynthesis 315 ]: Instead of trying to make predictions that can be tested by observation, cosmologists take observations that can be made, such as measurements of the abundances in Population II material, and try to determine the primordial abundances while knowing a priori what answer is needed to fit some particular model. They then take those derived abundances and adjust the model to match more closely so that circularity is completely guaranteed. In this way, the baryon density derived is too low to account for the subsequent large scale structure of the universe, and an ad hoc addition of cold, dark non-baryonic matter, cosmological constant must be introduced.

Less than $50 \%$ of the baryons predicted by the $\Lambda \mathrm{CDM}$ model to exist at low redshift have been found in some way in the galaxies or the intergalactic medium [316, 317, 318. Where is the rest of it? A second problem with baryons is that galaxies are observed to have a significantly smaller baryon fraction relative to the cosmic average 317,318. Tentative explanations of these missing baryons are given: a large fraction of the missing baryons may reside in the filaments of the cosmic web[319].

\subsection{Reionization epoch}

In Big Bang cosmology, reionization is the process that ionized the matter in the universe after the dark ages. As the majority of baryonic matter is in the form of hydrogen, reionization usually refers to the reionization of hydrogen gas. This occurred once objects started to condense in the early universe that were energetic enough to reionize neutral hydrogen. As these objects formed and radiated energy, the universe reverted from being neutral to once again being an ionized plasma. At that time, however, matter had been diffused by the expansion of the universe, and the scattering interactions of photons and electrons were much less frequent than before electron-proton recombination. Thus, a universe full of low density ionized hydrogen would remain transparent, as is the case today.

The presence of diffuse neutral hydrogen should produce an absorbing trough shortward of a QSO's Lyman-alpha emission line - the Gunn-Peterson effect. A hydrogen Gunn-Peterson trough was predicted to be present at a redshift $z \approx 6.1$. Indeed, a complete Gunn-Peterson trough at $z=6.28[320$. was discovered, which means that the Universe is approaching the end of the reionization epoch at $z_{r} \approx 6[320$. The quasar population is unlikely to provide enough photons to ionize the universe at $z \approx 6$ [321], even less for higher redshift, where there is a lower number of QSOs. At $z \approx 6$, the amount of absorption increases quickly with redshift (a tentative detection of a complete Gunn-Peterson trough), indicating that $z \approx 6$ is close to the end of the reionization epoch. However, the $z=6.5$ Lyman-alpha lines are not strongly 
suppressed by a neutral intergalactic medium, indicating that reionization was not largely complete at $z=6.5$ [322], and galaxies have been observed at much higher redshifts without the opacity features prior to the reionization, so the epoch of reionization was moved beyond those higher redshifts. An inhomogeneous reionization 320 is a possibility to explain the apparent disagreement of the different data.

Measurements of CMBR anisotropies give a reionization epoch $z_{r}=10.5 \pm$ 1.2 (with WMAP data[323]) or $z_{r}=8.8_{-1.2}^{+1.3}$ (with Planck data[324]). This might be indicative that reionization is likely to have been extended in time; otherwise, we would have a tension here.

It is thought that reionization might be due to light from massive stars. But this is still a problem for reionization at $z \approx 10$ because the measured star formation density is found to decrease too quickly with increasing redshift 325 326. Another exotic and ad hoc proposal to solve the problem uses, for instance, fast accretion shocks formed around the cores of the most massive haloes 327 .

\section{Formation of galaxies and dark matter problem}

\subsection{Large scale structure}

Like in all the other sections, there are other theories which explain the formation of voids and structure: radiation given off by primordial galaxies and QSOs 315]; galaxies that beget other galaxies 168; plasmas in the middle of magnetic fields and electric currents that create filaments, and this would explain the filamentary Universe 28, 328, 329, 330,331; the Quasi steady State Theory [332, which reproduces the observed two-point correlation function; and others.

Even if we ignore the alternative scenarios, we cannot say that everything is well undestood in the standard one. Caveats or open questions are still present. The standard theory would require large-scale homogeneity on scales of distance greater than a few tens of Mpc, and the distribution of galaxies and clusters of galaxies should be random on large scales. However, the departures from homogeneity claiming a fractal Universe and the regularity of structure are frequent. Pencil-beam surveys show large-scale structure out to distances of more than $1 \mathrm{Gpc}$ in both of two opposite directions from us. This appears as a succession of wall-like galaxy features at fairly regular intervals, with a characteristic scale of $128 h^{-1} \mathrm{Mpc} 333$, the first of which, at about 130 Mpc distance, is called "The Great Wall". Several such evenly-spaced "walls" of galaxies have been found 334]. The apparent lack of periodicity in other directions led to the initial report being regarded as a statistical anomaly 335, but a reconfirmation of a $(120 \pm 15) h^{-1} \mathrm{Mpc}$ periodicity for clusters of galaxies came after that (e.g. 336). An analysis of the distribution $N(z)$ of photometric redshifts in a grid of the deep fields reveals the possible existence of super-large 
structures with a contrast $d N / N \sim 50 \%$ and tangential and radial dimensions of about $1000 \mathrm{Mpc}$ 337,338.

Some observations show large scale inhomogeneities and peculiar velocities typical of a fractal on scales up to at least $100 \mathrm{Mpc}$, so the density fluctuations inside the fractal inhomogeneity cell will lead to a strong disturbance of pure Friedmann behaviour $339,340,341,342,343$. Hence, the Universe is not close to the Friedman-Lemaitre-Robertson-Walker on scales less than $100 \mathrm{Mpc}$. Statistical differences from homogeneous distribution were even found out to a scale of at least $200 \mathrm{Mpc} 344$. Structure is dominated by filaments and voids on this level 345 and by large velocity flows relative to the cosmological background 346]. However, other observations suggest the opposite conclusion: a striking linearity of the Hubble law in the distance range between 2 and 25 Mpc 347, 165, 348, 349. Beside the paradox, parameters such $H_{0}, q_{0}$ and $\Omega_{0}$ are determined without knowing on which scales the radial motion of galaxies and clusters of galaxies relative to us is completely dominated by the Hubble flow 350]. Most cosmologists appeal to the highly isotropic character of the microwave background as one of the principal justifications for assuming that the Universe is homogeneous on large scales. By itself, the fact that some observer sees isotropic background radiation is inconclusive, for this can be true in a static inhomogeneous universe, as well as in a spherically symmetric inhomogeneous universe where we are near a centre of symmetry [350. So an open question is apparently present.

There is streaming inconsistent with the Local Group absolute space velocity inferred from the CMBR dipole anisotropy 351]. The standard model interprets this as the existence of a puzzling group flow of galaxies relative to the microwave radiation on scales of at least $130 \mathrm{Mpc}$. Earlier, the existence of this flow led to the hypothesis of a "Great Attractor" pulling all these galaxies in its direction. But in newer studies, no backside infall was found on the other side of the hypothetical feature, possibly because it is difficult to observe given that it is behind the Galactic plane. But if there were no attractor, the only alternative within the standard model to the apparent result of the large-scale streaming of galaxies would be that we are almost at rest, and that the microwave radiation is in motion relative to us. Either way, this result would be a trouble for the orthodox interpretation.

The local streaming motions of galaxies are too high for a finite universe that is supposed to be everywhere uniform. In the early 1990s, we learned that the average redshift for galaxies of a given brightness differs on opposite sides of the sky 352,353,354, 355. There is also the Bullet Cluster problem: the observed large relative velocity of the two interacting galaxy clusters is not accountable for in the standard cosmological model 356 357]. There are anomalously large bulk flows on scales of $100 \mathrm{Mpc} / \mathrm{h}$ and beyond, which challenge the standard $\Lambda \mathrm{CDM}$ concordance model as well as a large class of competitive models of dark energy and modified gravity 358. Results from the measurement of large-scale peculiar velocities of clusters of galaxies using the largest all-sky X-ray cluster catalogue combined to date and CMBR data 359 find a strong and coherent bulk flow on scales out to at least $>300 h^{-1} \mathrm{Mpc}$. 
This flow is difficult to explain by gravitational evolution within the framework of the concordance $\Lambda \mathrm{CDM}$ model. The results are confirmed with recent "Planck?' CMBR data 360, which show that the observed dipole of kinetic Sunyaev-Zel'dovich effect that gives place to the dark flow is not contaminated by thermal Sunyaev-Zel'dovich effect. This dipole was not found with SNIa peculiar velocities (once the Hubble flow is removed) but the sample is quite inhomogeneous. Possible interpretations: i) an intrinsic dipole of CMBR in the last scattering surface (but the prediction of inflation gives a much lower dipole); ii) the flow is real. Both interpretations are problematic.

Another matter is the void problem: the Local Void is observed to be too empty in comparison to the standard cosmology predictions 361,362. Standard cosmology also predicts more dwarf or irregular galaxies in voids than observed 363].

Then there is the problem with profiles of Cluster Haloes: ACDM predicts a shallow low concentration and density profiles in contrast to observations that indicate a denser high concentration cluster haloes 363 .

Baryonic Acoustic Oscillations (BAO) found in the Large Scale Structure 364 are a good point in favour of the standard model. But the BAO peak position depends slightly on the enrironment of galaxies, whether in superclusters or not, which is not predicted by the standard model 365]. And analyses of the Alcock-Paczynski test using Baryonic Acoustic BAO peaks 125 exclude $\Lambda$ CDM within $98 \%$ C.L.

\subsection{Periodicity in large-scale structure}

In a homogeneous and isotropic universe we also expect the redshift distribution of extragalactic objects to approximate to a continuous and aperiodic distribution. However, Tifft 366, 367,368, affirmed that there was a periodicity of $70-75 \mathrm{~km} / \mathrm{s}$ in the redshift of the galaxies. In an improved correction for the optimum solar vector, the periodicity is found to be $37 \mathrm{~km} / \mathrm{s}$ (which includes Tifft's result for twice this velocity) with a probability of finding this period by chance of $2.7 \times 10^{-5} 369$. A galactocentric periodicity of $37 \mathrm{~km} / \mathrm{s}$ was confirmed to exist in the redshift distribution of nearby spiral galaxies 370 . The periodicity is seen in all the datasets examined. Using the distance information, there is some evidence that the periodicity is not strictly with respect to a fixed galactocentric velocity vector, but is referred to a vector that varies with increasing distance.

A periodicity with $\Delta z=0.031$ or 0.062 was also found for the QSOs 371 , 372 and a 0.089 periodicity in $\log _{10}(1+z)$ [370. Other works have also found similar periodicities $373,374,375,376,377,378$. However, other authors $379,380$. $381,382,383$ find no significant periodicity.

An oscillating scalar field model 384 with mass with particles of mass $3.2 \times 10^{-31} \mathrm{eV}$ has been proposed to explain the the periodic structure in galaxy counts vs. redshift. Also, in 1990 the Finnish physicist Ari Lehto had come up with a general formula for the quantization of physical quantities, 
without knowing of Tifft's astronomical studies[22](§9.5.2). He found that his formula describes well the redshift periods 36 and $72 \mathrm{~km} / \mathrm{s}$ that Tifft has derived. If the energy of any photon is quantized, as Lehto suggests, then so are the frequencies, hence the redshifts of light would occur at preferred values. Lehto had searched for a common rule for the properties of the micro- and the macroworld and he found that the ratios of the quantities involving lengths or energies may be expressed as $2^{n / 3}(\mathrm{n}=1,2,3, \ldots)$. What he did was to take the ratios of observed values of various stationary discrete systems (such as Planck energy/electron rest mass etc.) and he noted that the exponents of two thus obtained seemed to group near $0,1 / 3$ and $2 / 3$ [385]. In a more extensive study 386], involving over 40 quantities, it was shown that it is quite unlikely that the groupings could be due to chance. The pattern is best revealed by quantities that have been most accurately measured (laboratory physics), but it seems to be visible also for cosmic quantities. Lehto's system has the Planck units as natural starting points for making up the physical world. The Planck scale is absolute, based on constants of nature. For example, the observed temporary periods take values $t=t_{P l} 2^{n / 3}$, and the electron mass is obtained as $m_{e}=m_{P l} 2^{227 / 3}$. The redshift steps of about 72 and $36 \mathrm{~km} / \mathrm{s}$ would come from $V=2^{n / 3} c$ where $\mathrm{n} / 3$ is 12 and 13 .

\subsection{Antimatter and inflation}

Protons do not decay, as far as we know from particle physics experiments until now. Therefore, the Universe should be made up equally of matter and antimatter according to the standard model. Matter dominates the present universe apparently because of some form of asymmetry, such as $\mathrm{CP}$ violation asymmetry, that caused most anti-matter to annihilate with matter, but left much residual matter. Experiments are searching for evidence of this asymmetry, so far without success. Other galaxies cannot be antimatter because that would create a matter-antimatter boundary with the intergalactic medium that would create gamma rays, which are not seen 387. Another mystery of the standard model.

The introduction of inflation into the standard theory is another sign of the weakness of the model. The inflation necessary to explain a flat Universe is very improbable 388 . And inflation has no predictive power because anything may happen with inflation. A theory that can predict anything is a theory that predicts nothing. There are indeed $\sim 1000$ models of inflation 389].

5.4 Dark matter and the inconsistencies of the theory at galactic scales

Zwicky 390 paper in 1933 on dark matter in rich clusters proposed an application of the virial theorem to these data and it gave a mass-to-light ratio of $\sim 50$ in solar units. Kahn \& Woltjer 391 in 1959 determined the mass of the Local Group and obtained a mass-to-light ratio of 43 in solar units. In the 
'50s, Page 392, 393. also found that pairs of elliptical galaxies had a mass-tolight ratio of $66 \pm 14$ in solar units. This showed that such binaries must have massive envelopes or be embedded in a massive common envelope. Similar results were obtained in the ' 50 s from 26 binary galaxies by Holmberg 394 . Babcock 395 first showed in 1939 the need for dark matter for an individual galaxy, by measuring the rotation curve of the outer regions of M31. However, the majority of astronomers did not become convinced of the need for dark matter in galaxies until the publication of works in the '70s on the stability of galactic discs by Ostriker \& Peebles 397, the paper in which Ostriker et al. 398. showed that the mass of spiral galaxies increases almost linearly with radius to nearly $1 \mathrm{Mpc}$, and rotation curves by Vera Rubin 396. Once again, this shows the typical mentality of astrophysicists: accepting facts only when there is a theory supporting it with an explanation, a not-so-empirical approach that dominates the development of cosmology.

That there is some dark matter, either baryonic or non-baryonic, is clear, but how much, and what is its nature? The success of the standard model in converting a hypothesis into a solid theory depends strongly on the answer to these open questions. Stellar and cold gas in galaxies sum to baryonic matter content that is $8_{-5}^{+4} \%$ of the total amount of the predicted Big Bang baryonic matter 399]. Where is the rest of the baryonic material? From here stems another open question. Baryonic matter amounts to only around a tenth of the total amount of matter 400 . What is the nature of the putative non-baryonic dark matter required to achieve the current value of $\Omega_{m} \approx 0.3$ ?

Current CDM models predict the existence of dark matter haloes for each galaxy whose density profile falls approximately as $r^{-2}$, although the original idea 401 concerning hierarchical structures with CDM, which gave birth to the present models, was that the dark matter was distributed without internal substructure, more like a halo with galaxies than galaxies with a halo 402, something similar to the scenario in Refs. [403,404.

Some authors have been led to question the very existence of this dark matter on galactic scales since its evidence is weak [402,405, 406, 407] and the predictions do not fit the observations: CDM has a "small scale crisis" since there are some features of the galaxies that are very different from the predictions of the cosmological model. Nonetheless, many researchers are eagerly trying to find solutions that make data and model compatible, assuming a priori that the model "must be" correct. Some of the problems are the following.

There is a problem with a observed lower density of the halo in the inner galaxy than predicted. Profiles of Galaxy Haloes: 1 CDM predicts halo mass profiles with cuspy cores and low outer density, while lensing and dynamical observations indicate a central core of constant density and a flattish high dark mass density outer profile 363 . The possible solutions of core-cusp problem without abandoning the standard model are: bar-halo friction, which reduces the density of the halo in the inner galaxy 408; haloes around galaxies may have undergone a compression by the stellar disc 409] or/and suffered from the effects of baryonic physics 410]. 
Another problem is that the predicted angular momentum is much less than the observed one. Binney et al. 411] claim that the problem of an excess of predicted dark matter within the optical bodies and the fact that the observed discs are much larger than expected can be solved if a considerable mass of low angular momentum baryons is ejected (massive galactic outflows) and the discs are formed later from the high angular momentum baryons which fell in the galaxy. The conspiracy problem is also solved if the ejection begins only once $M_{\text {baryons }}(r) \sim M_{\text {dark matter }}(r)$. Another solution within the standard cosmological model for the angular momentum problem is the tidal interaction of objects populating the primordial voids together with the Coriolis force due to void rotation 412 .

The enclosed dynamical mass-to-light ratio increases with decreasing galaxy luminosity and surface brightness, which is not predicted by dark matter scenarios 413 .

Galaxies dominate the halo with little substructure whereas the model predicts that galaxies should be scaled versions of galaxy clusters with abundant substructure 414, 415. Also, the distribution of satellites is in a plane, incompatible with $\Lambda \mathrm{CDM}$ 415, 416, 417. The current standard model of cosmology requires the Dual Dwarf Galaxy Theorem to be true 418. According to this theorem, two types of dwarf galaxies must exist: primordial dark-matter dominated (type A) dwarf galaxies, and tidal-dwarf and ram-pressure-dwarf (type B) galaxies void of dark matter. In the model, type A dwarfs are distributed approximately spherically following the shape of the host galaxy dark matter halo, while type B dwarfs are typically correlated in phase-space. Type B dwarfs must exist in any cosmological theory in which galaxies interact. Only one type of dwarf galaxy is observed to exist: type B dwarfs lie on the baryonic Tully-Fisher relation, which is, however, defined by the putative type A dwarfs. Young and old type B dwarfs coincide with $\mathrm{dE}$ galaxies in radii and masses, and $\mathrm{dE}$ galaxies are observed to be baryon-dominated although they are popularly thought to be of putative type A. Kroupa 418] says that these are arguments against the standard model in which one cannot make the typical rebuff of incompleteness of knowledge of baryonic physics. Furthermore, there is a correlation between bulge mass and the number of luminous satellites in tidal streams 415, 419] that is not predicted by the standard model, and it is predicted by models of modified gravity without dark matter. The disc of satellites and bulge-satellite correlation suggest that dissipational events forming bulges are related to the processes forming phase-space correlated satellite populations. These events are well known to occur since in galaxy encounters energy and angular momentum are expelled in the form of tidal tails, which can fragment to form populations of tidal-dwarf galaxies and associated star clusters. If Local Group satellite galaxies are to be interpreted as Tidal Dwarf galaxies then the substructure predictions of the standard cosmological model are internally in conflict 415 .

Perhaps, that most severe caveat to retain the hypothesis of non-baryonic cold dark matter is that, after a long time looking for it, it has not yet been found, although non-discovery does not mean that it does not exist. Microlens- 
ing surveys 420] constrain the mass of the halo in our Galaxy in the form of dim stars and brown dwarfs to be much less than that necessary for dark matter haloes. Some observations are inconsistent with the dominant dark matter component being dissipationless [421]. Neither are black hole haloes a consistent scenario 422. The nature of dark matter has been investigated 423] and there are no suitable candidates. The latest attempts to search for exotic particles have also finished without success. For instance, it has been attempted to detect neutralinos with the MAGIC and HESS Cerenkov telescope systems for Very High Energy Gamma Rays through their Cherenkov radiation, but so far without success and only some emission associated with the Galaxy has been found 424. Dwarf galaxies are expected to have high ratios of dark matter and low gamma ray emission due to other astrophysical processes so the search is focussed on these galaxies, but without positive results. As usual, the scientists involved in these projects justify their failure by the fact the detectors may still be 3-4 orders of magnitude below of the possible flux of gamma rays emitted by Dark Matter 425] and ask for more money to continue to feed their illusions.

Note also that some other dynamical problems in which dark matter has been claimed as necessary can indeed be solved without dark matter: galactic stability [426, warp creation 404, rotation curves 402,427]. Velocities in galaxy pairs and satellites might measure the mass of the intergalactic medium filling the space between the members of the pairs 403, 404 rather than the mass of dark haloes associated with the galaxies. Also the dark matter necessary to solve many problems may be baryonic: positively charged, baryonic (protons and helium nuclei) particles 428, which are massive and weakly interacting, but only when moving at relativistic velocities; simple composite systems that include nucleons but are still bound together by comparable electric and magnetic forces 429], making up a three-body system "tresinos" or four body system "quatrinos"; antiparticles which have negative gravitational charge 430, etc.

\subsection{Dark energy and the cosmological constant or quintessence}

The question of the cosmological constant 431, Einstein's biggest blunder considered now to be not such a blunder, is very amusing. Two decades ago, most cosmologists did not favour the scenarios dominated by the cosmological constant 432 . In the eighties, the cosmological constant was many times disregarded as an unnecessary encumbrance, or its value was set at zero 433, and all the observations gave a null or almost null value. However, since other problems in cosmology have risen, many cosmologists at the beginning of the '90s realized that an $\Omega_{\Lambda}=0.70-0.80$ could solve many problems in CDM cosmology 434. Years later, evidence for such a value of the cosmological constant began to arrive. A brilliant prediction or a prejudice which conditions the actual measurements? Another open question. 
One measurement of the cosmological constant comes nowadays from supernovae, whose fainter-than-expected luminosity in distant galaxies can be explained with the introduction of the cosmological constant. It was criticized as being due possibly to intergalactic dust 435, 436, 437]. The presence of grey dust is not necessarily inconsistent with the measure of a supernova at $z=1.7$ (SN 1997ff) 436. Dimming by dust along the line of sight, predominantly in the host galaxy of the SN explosion, is one of the main sources of systematic uncertainties 438. Also, there was an underestimate of the effects of host galaxy extinction: a factor which may contribute to apparent faintness of high- $z$ supernovae is the evolution of the host galaxy extinction with $z$ 439]; therefore, with a consistent treatment of host galaxy extinction and the elimination of supernovae not observed before maximum, the evidence for a positive $\Lambda$ is not very significant. Fitting the corrected luminosity distances (corrected for internal extinctions) with cosmological models Balazs et al. 99. concluded that the SNIa data alone did not exclude the possibility of the $\Lambda=0$ solution.

Type Ia Supernovae also possibly have a metallicity dependence and this would imply that the evidence for a non-zero cosmological constant from the SNIa Hubble Diagram may be subject to corrections for metallicity that are as big as the effects of cosmology [440]. The old supernovae might be intrinsically fainter than the local ones, and the cosmological constant would not be needed 441. As a matter of fact, some cases, such as SNLS-03D3bb, have an exceptionally high luminosity 442 . Claims have been made about the possible existence of two classes of Normal-Bright SNe Ia 443. If there is a systematic evolution in the metallicity of SN Ia progenitors, this could affect the determination of cosmological parameters. This metallicity effect could be substantially larger than has been estimated previously and could quantitatively evaluate the importance of metallicity evolution for determining cosmological parameters 444. In principle, a moderate and plausible amount of metallicity evolution could mimic a $\Lambda$-dominated, a flat Universe in an open, $\Lambda$-free Universe. However, the effect of metallicity evolution appears not to be large enough to explain the high- $z$ SNIa data in a flat Universe, for which there is strong independent evidence, without a cosmological constant.

Furthermore, our limited knowledge of the SN properties in the U-band has been identified as another main source of uncertainty in the determination of cosmological parameters 438. And the standard technique with SNe Ia consists in using spectroscopic templates, built by averaging spectra of well observed (mostly nearby) SNe Ia. Thus, the uncertainty in K-corrections depends primarily on the spectroscopic diversity of SNe Ia.

Even if we accept the present-day SN Ia analyses as correct and without any bias or selection effect, other cosmologies may explain the apparent cosmic acceleration of SNe Ia without introducing a cosmological constant into the standard Einstein field equation, thus negating the necessity for the existence of dark energy 445. There are four distinguishing features of these models: 1) the speed of light and the gravitational "constant" are not constant, but vary with the evolution of the universe, 2) time has no beginning 
and no end, 3) the spatial section of the universe is a 3-sphere, and 4) the universe experiences phases of both acceleration and deceleration. An inhomogeneous isotropic universe described by a Lemaitre-Tolman-Bondi solution of Einstein's fields equations can also provide a positive acceleration of the expansion without dark energy 446. Quasi-Steady-State theory predicts a decelerating universe at the present era, it explains successfully the recent $\mathrm{SNe}$ Ia observations 447. Carmeli's cosmology fits data for an accelerating and decelerating universe without dark matter or dark energy 448. Thompson 449 ] used available measurement for the constrainst on the variation the proton to mass electron with redshift, and with $\frac{\Delta \alpha}{\alpha}=7 \times 10^{-6}$ he finds that almost all of the dark energy models using the commonly expected values or parameters are excluded. A static Universe can also fit the supernovae data without dark energy 102, 103, 104, 105, 106.

There are other sources of $\Omega_{\Lambda}$ measurement: the anisotropies of the microwave background radiation (see my doubts on the accuracy of using them to determine cosmological parameters in 3.5, for instance. In the last two decades, a lot of proofs have been presented to the community to convince us that the definitive cosmology has $\Omega_{\Lambda} \approx 0.7$, which is surprising taking into account that in the rest of the history of the observational cosmology proofs have been presented for $\Omega_{\Lambda} \approx 0$. Furthermore, recent tests indicate that other values are available in the literature. For instance, from the test angular size vs. redshift for ultracompact radio sources, it is obtained that $\Lambda$ has the opposite sign [450]. Using the brightest galaxies in clusters, with two independent samples, the fit in the Hubble diagram is compatible with a Universe without acceleration instead of $\Omega_{\Lambda}=0.7$ [11].

Moreover, the actual values of $\Omega_{\Lambda}$ have some consistency problem in the standard scenario of the inflationary Big Bang. The cosmological constant predicted by quantum field theory has a value much larger than those derived from observational cosmology. This is because the vacuum energy in quantum field theory takes the form of the cosmological constant in Einstein's equations. If inflation took place at the GUT epoch, the present value would be too low by a factor $\sim 10^{-108}$, and if the inflation took place at the quantum gravity epoch, the above factor would be lower still at $\sim 10^{-120}$ [451.

The standard model has some surprising coincidences. There is the coincidence that now the deceleration of the Hubble flow is compensated by the acceleration of the dark energy 452 (Ch. 3). The average acceleration throughout the history of the Universe is almost null 453 . Another apparent paradox is that the Big Bang Model assumes that the cosmic fluid is not only continuous but also homogeneous and isotropic, intrinsically corresponding to zero pressure and hence zero temperature. Therefore, the ideal Big Bang Model cannot describe the physical universe as having pressure, temperature, and radiation. Consequently, the physical universe may comprise matter distributed in discrete non-continuous lumpy fashion (as observed) rather than in the form of a homogeneous continuous fluid. The intrinsic absence of pressure in the "Big Bang Model" also rules out the concept of "Dark Energy", according to some opinions 454. 
Again, everything is far from being properly understood and with wellconstrained parameters.

5.6 Distant galaxies, evolution, and the age of galaxies

The Big Bang requires that stars, QSOs, and galaxies in the early universe be "primitive", meaning mostly metal-free, because it requires many generations of supernovae to build up the metal content in stars. But the evidence shows the existence of even higher than solar metallicities in the "earliest" QSOs and galaxies $320,321,455$. The iron-to-magnesium ratio increases at higher redshifts 456. And what is even more amazing: there is no evolution of some line ratios, including iron abundance 457,458,459,460, between $z=6.5$ and $z=0$. There is no evolution of CaII absorbers with redshift 461](Fig. 9). The amount of dust in high redshift galaxies and QSOs is also much higher than expected 462 .

From the comparison with a synthesis model, colours of some very red galaxies at $z>2.5$ could be related to ages of a stellar population in passively evolving galaxies older than $1 \mathrm{Gyr}$ on average 463 . They turn out to be massive galaxies (stellar masses of $\sim 10^{11} \mathrm{M}_{\odot}$ ) that where formed on average when the Universe was very young $(<1 \mathrm{Gyr})$, assuming the standard cosmological parameters. Some early-type massive extremely red objects with $z \sim 1.4$ [464, 465. are also very old: a galaxy with $z=1.22 \pm 0.05$ has an average stellar population age of $5.0 \pm 0.1 \mathrm{Gyr}$ [464], again in the limit of the age of the Universe at this redshift $(5.3 \pm 0.2 \mathrm{Gyr})$. It is clear from this information that the formation of very massive elliptical galaxies should take place at very high redshifts. More distant $(z>2)$ red galaxies have been analysed 466 with observations that provide rest-frame UV-to-NIR photometry (derived from 0.3-8.0 $\mu \mathrm{m}$ photometry) and it has been found that three of the 14 red galaxies were indeed old galaxies with Single Stellar Population best-fit ages 2.6 Gyr at $z=2.7 \pm 0.4,3.5 \mathrm{Gyr}$ at $z=2.3 \pm 0.3$, and 3.5 Gyr at $z=2.3 \pm 0.3$ respectively, representative of the average age of the stellar populations, so the oldest stars must be still older. The age of the Universe in the standard model at both redshifts of $2.7 \pm 0.4$ and $2.3 \pm 0.3$ is $2.5 \pm 0.5$ Gyr and $3.0 \pm 0.4$ Gyr respectively. We are again with the case of galaxies as old as the Universe. Toft et al.467] fitted synthesis models to the spectra of red compact galaxies with ages 5.5, 3.5 and 1.7 Gyr for redshifts 1.2, 1.9 and 3.4 (ages of the Universe respectively: 5.4, 3.6, $2.0 \mathrm{Gyr}$ ), in the limit. Galaxies at very high redshift $(z \sim 4)$ have been analysed 468 to find, with optical, near-infrared and mid-infrared surveys, an important ratio of old $(\sim 1$ Gyr or older $)$ and massive $\left(\sim 10^{11} \mathrm{M}_{\odot}\right)$ galaxies. With similar techniques evidence has been presented for 11 massive and evolved (0.2-1.0 Gyr) galaxies at redshifts $4.9 \leq z \leq 6.5$ (photom. redshifts) 469. This is what has been called the impossible early galaxy problem, according to which observations find several orders of magnitude more very massive haloes at very high redshift than predicted, implying that these massive galaxies formed impossibly early [470]. 
These results are at odds with semianalytical $\Lambda$ CDM models, which claim that very massive galaxies were formed much later 471, although along the line suggested by the "downsizing" scenario of galaxy formation.

In view of all this evidence and the tensions within the results expected a priori, orthodox cosmologists have claimed that star formation began very early and produced metals up to the solar abundance quickly, in a few hundred Myr, and in which most massive galaxies assembled substantial amounts of their stellar content rapidly (in 1-2 Gyr) beyond $z \sim 3$ in very intense star formation episodes 472. This idea may find some support in observations such as the discovery of a massive starburst galaxy at $z=6.34$ [73], with mass around $3 \times 10^{11}$ solar masses. A "maximum starburst" converts the gas into stars at a rate more than 2000 times that of the Milky Way, a rate among the highest observed at any epoch. All well and good, but this scenario is at odds with the predictions of the galaxy formation in hierarchical scenarios of $\Lambda \mathrm{CDM}$ cosmological model, so the hypothesis which served to explain the large scale structure succesfully is failing here.

\section{Conclusions}

There are many tenets of the standard cosmological theory that are being cast into doubt in a wide-ranging literature. Certainly, many of the references that I have cited in this review may contain wrong or uncertain arguments and results, and further research is needed to confirm or refute their statements, but it is good that we do not forget them and continue to think about these controversial topics.

Note that I am not defending any specific idea of the cosmos here: neither the correctness nor the wrongness of Big Bang. I am just presenting a number of sceptical arguments expressing certain doubts on the validity of the standard cosmology that are found in a wide literature. We must also admit that alternative theories are not at present as competitive as the standard model in cosmology in terms of giving better explanations. If they were more developed, there is a possibility that they might compete in some aspects with the Big Bang theory, but efforts are made in the present-day scientific community to avoid their development 1 . In any case, one advantage of alternative theories is that their study may lead to new useful cosmological tests, even if the theories themselves were not viable, for instance the redshift-angular size test in connection with his studies of the Steady State theory proposed by Hoyle 113 . We need unquestionable facts, free from interpretation (as Lemaître or Hubble did when they discovered the expansion) and good physicists to propose physical theories (Einstein, Gamow, etc.). There are so many works on cosmology, that it is more and more difficult to propose a theory without discussing all previous studies. This paper may be of interest in this sense. My hope is that the reader of this review may find it useful and inspiring in order to carry out new and challenging research, rather than the usual boring and conformist attitude in present-day cosmology. Or can you say, after reading this paper, 
that everything in the standard model is clear and well established and the only remaining mission is to refine the measurement of certain parameters?

Acknowledgements Thanks are given to Fulvio Melia and the two anonymous referees for comments on a draft of this paper that helped to improve it. Thanks are given to Terence J. Mahoney for proof-reading of the text.

\section{References}

1. López-Corredoira, M.: Non-standard models and the sociology of cosmology. Studies in History and Philosophy of Modern Physics, 46-A, 86-96 (2014)

2. Einstein, A.: Kosmologische Betrachtungen zur allgemeinen Relativitätstheorie. In: Sitzungsberichte der Königlich Preussischen Akademie der Wissenschaften, pp. 142-152. Berlin (1917)

3. Narlikar, J.V., Arp, H.C.: Flat spacetime cosmology - A unified framework for extragalactic redshifts. Astrophys. J., 405, 51-56 (1993)

4. Boehmer, C.G., Hollenstein, L., Lobo, F.S.N.: Stability of the Einstein static universe in f(R) gravity. Phys. Rev. D, 76, id. 084005 (2007)

5. Van Flandern, T.: Is the gravitational constant changing? In: Taylor, B. N., Phillips, W. D. (eds.) Precision Measurements and Fundamental Constants II (National Bureau of Standards Special Publication 617), pp. 625-627 (1984)

6. Troitskii, V. S.: Physical constants and evolution of the universe. Astrophys. Space Science, 139, 389-411 (1987)

7. Van Flandern, T.: Dark Matter, Missing Planets and New Comets. North Atlantic Books, Berkeley (1993)

8. Francis, M.J., Barnes, L.A. James, J.B., Lewis, G.F.: Expanding Space: the Root of all Evil? Publ. Astron. Soc. Australia, 24, 95-102 (2007)

9. Baryshev, Yu.V.: Expanding space: the root of conceptual problems of the cosmological physics, In: Baryshev, Yu. V., Taganov, I. N., Teerikorpi, P. (eds.) Practical Cosmology, 1, pp. 20-30. TIN, St.-Petersburg (2008)

10. Feynman, R.P., Morinigo, F.B., Wagner, W.G.: Feynman lectures on gravitation. Addison-Wesley, Reading, MA (1995)

11. Baryshev, Yu.V.: Field fractal cosmological model as an example of practical cosmology approach In: Baryshev, Yu. V., Taganov, I. N., Teerikorpi, P. (eds.) Practical Cosmology, 1, pp. 60-67. TIN, St.-Petersburg (2008)

12. Bondi, H.: Cosmology (2nd. edition). Cambridge Univ. Press, London (1961)

13. Lemaître, G.: Un Univers homogène de masse constante et de rayon croissant rendant compte de la vitesse radiale des nébuleuses extra-galactiques, Annales de la Société Scientifique de Bruxelles A47, 49-59 (1927). Translated into English in: Expansion of the universe, A homogeneous universe of constant mass and increasing radius accounting for the radial velocity of extra-galactic nebulae. Mon. Not. R. Astron. Soc. 91, 483-490 (1931)

14. Hubble, E. P.: A Relation between Distance and Radial Velocity among Extra-Galactic Nebulae, Proc. US Nat. Acad. Sci. 15, 168-173 (1929)

15. Narlikar, J. V.: Noncosmological redshifts. Space Science Reviews, 50, 523-614 (1989)

16. Baryshev, Yu.V., Sylos Labini, F., Montuori, M., Pietronero, L.: Facts and ideas in modern cosmology Vistas in Astronomy, 38, 419-500 (1994)

17. Reboul, H.J.: Untrivial redshifts - A bibliographical catalogue. Astron. Astrophys. Supp. Ser., 45, 129-144 (1981)

18. Zwicky, F.: On the Red Shift of Spectral Lines through Interstellar Space. Proc. N. A. S., 15, 773-779 (1929)

19. Zwicky, F.: Morphological Astronomy. Springer, Berlin (1957)

20. Steinbring, E.: Are High-Redshift Quasars Blurry? Astrophys. J., 655, 714-717 (2007)

21. Roberts, M.S.: The gaseous content of galaxies (survey Lecture). In: Evans, D.S. (ed.) External Galaxies and Quasi-Stellar Objects (IAU Symp. 44), p. 12. Reidel, Dordrecht (1972) 
22. Baryshev, Yu.V., Teerikorpi, P.: Fundamental Questions of Practical Cosmology. Springer Verlag, Dordrecht (2012)

23. Vigier, J.P.: Alternative interpretation of the cosmological redshift in terms of vacuum gravitational drag. In: Bertola, F., Madore, B., Sulentic, J. (eds.) New Ideas in Astronomy, pp. 257-274. Cambridge University Press, Cambridge (1988)

24. Gallo, C.: A New Red Shift Mechanism with Possible Applications to Astrophysical Problems Such as Quasars. International Journal of Theoretical Physics, 13, 417-418 (1975)

25. Moret-Bailly, J.: The Parametric Light-Matter Interactions in Astrophysics. In: Lerner, E. J., Almeida, J. B. (eds.), 1st Crisis in Cosmology Conference (AIP Conf. Ser. 822(1)), pp. 226-238. AIP, Melville (2006)

26. Varshni, Y.P.: The physics of quasars. Phys. Canada, 35, 11-17 (1979)

27. Laio, A., Rizzi, G., Tartaglia, A.: Quantum theory of frequency shifts of an electromagnetic wave interacting with a plasma. Phys. Rev. E, 55, 7457-7461 (1997)

28. Lerner, E. J.: The Big Bang never happened: a startling refutation of the dominant theory of the origin of the universe. Random House, Toronto (1991)

29. Brynjolfsson, A.: Redshift of photons penetrating a hot plasma. arXiv.org: astro$\mathrm{ph} / 0401420(2004)$

30. Ashmore, L.: Intrinsic Plasma Redshifts Now Reproduced In The Laboratory - a Discussion in Terms of New Tired Light. viXra.org: 1105.0010 (2011)

31. Weidner, H.: The Size and Energy Loss of a Wave Packet. viXra.org: 1408.0139 (2014)

32. Mamas, D. L.: An explanation for the cosmological redshift. Phys. Essays, 23, 326-329 (2010)

33. Wolf, E.: Invariance of the spectrum of light on propagation. Phys. Rev. Lett., 56, 1370-1372 (1986)

34. Roy, S., Kafatos, M., Datta, S.: Shift of spectral lines due to dynamic multiple scattering and screening effect: implications for discordant redshifts. Astron. Astrophys., 353, 1134$1138(2000)$

35. Joos, C., Lutz J.: Quantum redshift. Paper presented at the Crisis in Cosmology Conference-I, Moncao, Portugal, 23-25 June 2005

36. Crawford, D.: Curvature Cosmology. BrownWalker Press, Boca Raton (Florida, US) (2006)

37. Crawford, D.: Observational Evidence Favors a Static Universe (Part I). J. Cosmology, $13,3875-3946(2011)$

38. Crawford, D.: Observational Evidence Favors a Static Universe (Part III). J. Cosmology, 13, 4000-4057 (2011)

39. Bondi, H.: Spherically symmetrical models in general relativity. Mon. Not. R. Astron. Soc., 107, 410-425 (1947)

40. Baryshev, Yu.V.: Hierarchical Structure of Metagalaxy - Problem Review. Astrofizicheskie Issledovaniia Izvestiya Spetsial'noj Astrofizicheskoj Observatorii, 14, 24 (1981)

41. Baryshev, Yu.V.: On the Fractal Nature of the Large-Scale Structure of the Universe. Astron. Astrophys. Trans., 5, 15-23 (1994)

42. Broberg, H.: The Geometry of Acceleration in Space-Time: Application to the Gravitational Field and Particles. In: Rudnicki, K. (ed.) Gravitation, Electromagnetism and Cosmology: Toward a New Synthesis, p. 9. Apeiron, Montreal (2001)

43. Nesvizhevsky, V.V., Börner, H.G., Petukhov, A.K., et al.: Quantum states of neutrons in the Earth's gravitational field. Nature, 415, 297-299 (2002)

44. Ghosh, A.: Velocity Dependent Inertial Induction: A Possible Mechanism for Cosmological Red Shift in a Quasi Static Infinite Universe. J. Astrophys. Astron. 18, 449-454 (1997)

45. Barber, G.: A New Self Creation Cosmology. Astrophys. Space Sci., 282, 683-730 (2002)

46. Barber, G.: The Principles of Self Creation Cosmology and its Comparison with General Relativity. arXiv.org: gr-qc/0212111 (2002)

47. Barber G.: Resolving the Degeneracy: Experimental Tests of the New Self Creation Cosmology and a Heterodox Prediction for Gravity Probe B. Astrophys. Space Sci., 305, 169-176 (2006)

48. Barber, G.: The derivation of the coupling constant in the new Self Creation Cosmology. arXiv.org: gr-qc/0302088 (2003) 
49. Fischer, E.: Homogeneous cosmological solutions of the Einstein equation. Astrophys. Space Sci., 325, 69-74 (2010)

50. Bouvier, P., Maeder, A.: Consistency of Weyl's geometry as a framework for gravitation. Astrophys. Space Sci., 54, 497-508 (1978)

51. Lunsford, D.R.: Gravitation and electrodynamics over $\mathrm{SO}(3,3)$. Int. J. Theor. Phys. 43, 161-177 (2004)

52. Krasnov, K., Shtanov, Y.: Non-metric gravity: II. Spherically symmetric solution, missing mass and redshifts of quasars Classical and Quantum Gravity, 25, id. 5002 (2008)

53. Castro, C.: On Dark Energy, Weyl Geometry and Brans-Dicke-Jordan Scalar Field. vixra.org: 0901.0001 (2009)

54. Ivanov, M.A.: Another origin of cosmological redshifts. arXiv.org: astro-ph/0405083 (2004)

55. Ivanov, M.A.: Low-Energy Quantum Gravity Leads to Another Picture of the Universe In: Lerner, E.J., Almeida, J.B. (eds.) 1st Crisis in Cosmology Conference (AIP Conf. Ser. 822(1)), pp. 187-199. AIP, Melville (2006)

56. Roscoe, D.: Maxwells Equations: New Light on Old Problems. Apeiron, 13, 206-239 (2006)

57. Mosquera Cuesta, H.J., Salim, J.M., Novello,M.: Cosmological redshift and nonlinear electrodynamics propagation of photons from distant sources. arXiv.org: 0710.5188 (2007)

58. Maxwell, J.C.: A Treatise on Electricity and Magnetism, vol. II (1891). Reprinted at: Dover, New York (1954)

59. Monti, R.: The electric conductivity of background space. In: Kostro, L., Posiewnik, A. Pykacz, J., Zukowski, M. (eds.) Problems in Quantum Physics, Gdansky 87 - Recent and Future Experiments and Interpretations, p. 640. World Scientific, Singapore (1988)

60. Nernst, W. von: The Structure of the Universe in Light of our Research. Jules Springer, Berlin (1921)

61. Alfonso-Faus, A.: Mass-Boom Versus Big-Bang: An Alternative Model. In: Lerner, E.J., Almeida, J.B. (eds.) 1st Crisis in Cosmology Conference (AIP Conf. Ser. 822(1)), pp. 107-109. AIP, Melville (2006)

62. Alfonso-Faus, A.: The case for a non-expanding universe. arXiv.org: 0908.1539 (2009)

63. Urbanowski, K.: On a possible quantum contribution to the red shift. In: Baryshev, Yu.V., Taganov, I.N., Teerikorpi, P. (eds.) Practical Cosmology, 1, pp. 117-122. TIN, St.-Petersburg (2008)

64. Segal, I.E.: Mathematical Cosmology and Extragalactic Astronomy. Academic Press, New York (1976)

65. Segal, I.E., Zhou, Z.: Maxwell's Equations in the Einstein Universe and Chronometric Cosmology. Astrophys. J. Supp. Ser., 100, 307-324 (1995)

66. Hoyle, F., Narlikar, J.V.: A New Theory of Gravitation. Proc. Roy. Soc. London, A282, 191-207 (1964)

67. Narlikar, J.V.: Two astrophysical applications of conformal gravity. Ann. Phys., 107, $325-336(1977)$

68. Garaimov, V.I.: Time and Entropy. In: Holt, S.S., Reynolds, C. S. (eds.) The emergence of cosmic structure (AIP Conf. Proc. 666), pp. 361-364. AIP, Melville (2003)

69. Chen, C. S., Zhou, X. L., Man, B. Y., Zhang, Y. Q., Guo, J.: Investigation of the mechanism of spectral emission and redshifts of atomic line in laser-induced plasmas. Optik, 120, 473-478 (2009)

70. Nguyen H., Koenig, M., Benredjem, D., Caby, M., Coulaud, G.: Atomic structure and polarization line shift in dense and hot plasmas. Phys. Rev. A, 33(2), 1279-1290 (1986)

71. Mérat, P., Pecker, J.-C., Vigier, J.-P., Yourgrau, W.: Observed Deflation of Light by the Sun as a Function of Solar Distance. Astron. Astrophys., 32, 471-475 (1974)

72. Mérat, P., Pecker, J.-C., Vigier, J.-P.: Possible Interpretation of an Anomalous Redsbift Observed on the $2292 \mathrm{MHz}$ Line Emitted by Pioneer-6 in the Close Vicinity of the Solar Limb. Astron. Astrophys., 30, 167-174 (1974)

73. Marmet, P.: Red shift of spectral lines in the sun's chromosphere. IEEE Transactions on Plasma Science, 17(2), 238-244 (1989)

74. Dravins, D.: Photospheric spectrum line asymmetries and wavelength shifts. Ann. Rev. Astron. Astrophys., 20, 61-89 (1982)

75. Sandage, A.: The Change of Redshift and Apparent Luminosity of Galaxies due to the Deceleration of Selected Expanding Universes. Astrophys. J., 136, 319-333 (1962) 
76. Liske, J., Grazian, A., Vanzella, E., et al.: Cosmic dynamics in the era of Extremely Large Telescopes. Mon. Not. Roy. Astron. Soc., 386, 1192-1218 (2008)

77. Molaro, P., Levshakov, S.A., Dessauges-Zavadsky, M., D'Odorico, S.: The cosmic microwave background radiation temperature at $z_{\mathrm{abs}}=3.025$ toward QSO 0347-3819. Astron. Astrophys., 381, L64-L67 (2002)

78. Noterdaeme, P., Petitjean, P., Srianand, R., Ledoux, C. López, S.: The evolution of the cosmic microwave background temperature. Measurements of $T_{\mathrm{CMB}}$ at high redshift from carbon monoxide excitation. Astron. Astrophys., 526, id. L7, 4 pp. (2011)

79. Krelowski, J., Galazutdinov, G., Gnacinski, P.: CN rotational excitation. Astron. Nachrichten, 333, 627-633 (2012)

80. Sato, M., Reid, M.J., Menten, K.M., Carilli, C. L.: On Measuring the Cosmic Microwave Background Temperature at Redshift 0.89. Astrophys. J., 764, id. 132, 8 pp. (2013)

81. Luzzi, G., Génova-Santos, R.T., Martins, C.J.A.P., De Petris, M., Lamagna, L.: Constraining the evolution of the CMB temperature with SZ measurements from Planck data. Journal of Cosmology and Astroparticle Physics, 9, id. 011 (2015)

82. Goldhaber, G., Groom, D.E., Kim, A., et al.: Timescale Stretch Parameterization of Type Ia Supernova B-Band Light Curves. Astrophys. J., 558, 359-368 (2001)

83. Blondin, S., Davis, T.M., Krisciunas, K., et al.: Time Dilation in Type Ia Supernova Spectra at High Redshift. Astrophys. J., 682, 724-736 (2008)

84. Nobili, S., Goobar, A.: The colour-lightcurve shape relation of type Ia supernovae and the reddening law. Astron. Astrophys., 487, 19-31 (2008)

85. Brynjolfsson, A.: Plasma Redshift, Time Dilation, and Supernovas Ia. arXiv.org: astro$\mathrm{ph} / 0406437$ (2004)

86. Leaning, S. P.: New Analysis of Observed High Redshift Supernovae Data Show that A Majority Of SN1a Decay Lightcurves can be Shown to Favourably Compare with a non Dilated Restframe Template. In: Lerner, E.J., Almeida, J.B. (eds.) 1st Crisis in Cosmology Conference (AIP Conf. Ser. 822(1)), pp. 48-59. AIP, Melville (2006)

87. Ashmore, L.: Supernovae Ia Light Curves Show a Static Universe. vixra.org: 1207.0015 (2012)

88. Holushko, H.: Tired Light and Type Ia Supernovae Observations. vixra.org: 1203.0062 (2012)

89. LaViolette, P. A.: Subquantum kinetics: The Alchemy of Creation (4th ed.). Starlane Publ., Niskayana (NY, US) (2012)

90. Crawford, D.: No Evidence of Time Dilation in Gamma-Ray Burst Data arXiv.org: 0901.4169 (2009)

91. Hawkins, M. R. S.: On time dilation in quasar light curves. Mon. Not. Roy. Astron. Soc., 405, 1940-1946 (2010)

92. Dai, D.-C., Starkman, G.D., Stojkovic, B., Stojkovic, D., Weltman, A.: Using Quasars as Standard Clocks for Measuring Cosmological Redshift. Phys. Rev. Lett., 108, id. 231302 (2012)

93. Moresco, M., Cimatti, A., Jiménez, R., et al.: Improved constraints on the expansion rate of the Universe up to $z \sim 1.1$ from the spectroscopic evolution of cosmic chronometers. J. Cosmol. Astropart. Phys., 8, id. 6 (2012)

94. Moresco, M., Pozzetti, L., Cimatti, A., et al.: A $6 \%$ measurement of the Hubble parameter at $z \sim 0.45$ : direct evidence of the epoch of cosmic re-acceleration. J. Cosmol. Astropart. Phys., 5, id. 14 (2016)

95. López-Corredoira, M., Vazdekis, A., Gutiérrez, C.M., Castro-Rodríguez, N.: Stellar content of extremely red quiescent galaxies at $z>2$. Astron. Astrophys, accepted (2017)

96. LaViolette, P.A.: Is the universe really expanding? Astrophys. J., 301, 544-553 (1986)

97. Kowalski, M., Rubin, D., Aldering, G., et al.: Improved Cosmological Constraints from New, Old, and Combined Supernova Data Sets. Astrophys. J., 686, 749-778 (2008)

98. Wei, H.: Observational constraints on cosmological models with the updated long gamma-ray bursts. J. Cosm. Astropart. Phys., 8, id. 20 (2010)

99. Balázs, L.G., Hetesi, Zs., Regály, Zs., Csizmadia, Sz., Bagoly, Zs., Horváth, I., Mészáros, A.: A possible interrelation between the estimated luminosity distances and internal extinctions of type Ia supernovae. Astron. Nachrichten, 327, 917-924 (2006)

100. Podsiadlowski, P., Mazzali, P., Lesaffre, P., Han, Z., Förster, F.: The nuclear diversity of Type Ia supernova explosions. New Astron. Rev., 52, 381-385 (2008) 
101. Bogomazov, A. I., Tutukov, A. V.: Type Ia supernovae: Non-standard candles of the universe. Astron. Reports, 55, 497-504 (2011)

102. Sorrell, W.H.: Misconceptions about the Hubble recession law. Astrophys. Space Sci., 323, 205-211 (2009). Erratum: Astrophys. Space Sci., 323, 213 (2009)

103. Lerner, E. J.: Tolman Test from $z=0.1$ to $z=5.5$ : preliminary results challenge the expanding universe model. In: Potter, F. (ed.) Second Crisis in Cosmology Conference (ASP Conf. Ser. 413), pp. 12-23. ASP, S. Francisco (2009)

104. López-Corredoira, M.: Angular-size test on the expansion of the Universe. Int. J. Mod. Phys. D, 19, 245-291 (2010)

105. Farley, F.J.M.: Does gravity operate between galaxies? Observational evidence reexamined. Proc. R. Soc. A, 466, 3089-3096 (2010)

106. Marosi, L.A.: Hubble Diagram Test of Expanding and Static Cosmological Models: The Case for a Slowly Expanding Flat Universe. Advances in Astronomy, 2013, id. 917104 (2013)

107. Schwarz, D.J., Weinhorst, B.: (An)isotropy of the Hubble diagram: comparing hemispheres. Astron. Astrophys., 474, 717-729 (2007)

108. Hubble, E.P., Tolman, R.C.: Two Methods of Investigating the Nature of the Nebular Redshift. Astrophys. J., 82, 302-337 (1935)

109. Lubin, L.M., Sandage, A.: The Tolman Surface Brightness Test for the Reality of the Expansion. IV. A Measurement of the Tolman Signal and the Luminosity Evolution of Early-Type Galaxies. Astron. J., 122, 1084-1103 (2001)

110. Lerner, E.J.: Evidence for a Non-Expanding Universe: Surface Brightness Data From HUDF. In: Lerner, E.J., Almeida, J.B. (eds.) 1st Crisis in Cosmology Conference (AIP Conf. Ser. 822(1)), pp. 60-74. AIP, Melville (2006)

111. Andrews, T.B.: Falsification of the Expanding Universe Model. In: Lerner, E.J., Almeida, J.B. (eds.) 1st Crisis in Cosmology Conference (AIP Conf. Ser. 822(1)), pp. 3-22. AIP, Melville (2006)

112. Lerner, E.J., Falomo, R., Scarpa, R.: UV surface brightness of galaxies from the local Universe to $z \sim 5$. Int. J. Mod. Phys. D, 23, id. 1450058 (2014)

113. Hoyle, F.: The relation of radio astronomy to cosmology. In: Bracewell, R.. N. (ed.) Radio Astronomy (IAU Symp. 9), pp. 529.-533 (1959)

114. Kapahi, V.K.: The angular size-redshift relation as a cosmological tool. In: Hewitt, A., Burbidge, G., Fang, L.-Z. (eds.) Observational Cosmology (IAU Symp. 124), pp. 251-265. Reidel, Dordrecht (1987)

115. Andrews, T.B.: Derivation of the Hubble Redshift and the Metric in a Static Universe. In: Lerner, E.J., Almeida, J.B. (eds.) 1st Crisis in Cosmology Conference (AIP Conf. Ser. 822(1)), pp. 123-143. AIP, Melville (2006)

116. Nabokov, N.V., Baryshev, Yu.V. Classical cosmological tests for galaxies of the Hubble Ultra Deep Field. Astrophys. Bull. 63, 244-258 (2008)

117. Lerner, E.: Surface Brightness of Galaxies and the Evidence Against the Concordance Model. Paper presented at the Observational anomalies challenging the Lambda-CDM cosmological model (Special Session 2, EWASS 2015), Tenerife, Spain 22 June 2015. http://www.iac.es/galeria/martinlc/EWASS2015/1339.pdf

118. Disney, M.J., Lang, R.H.: The galaxy ancestor problem. Mon. Not. R. Astron. Soc., 426, 1731-1749 (2012)

119. Valtonen, M., Nilsson, K., Kotilainen, J., Jaakkola, T.: Double radio sources as standard rods of testing cosmological models. In: Proc. of the 25th Annual Conf. of the Finnish Phys. Soc. Oulu Univ., Oulu (1991)

120. Nilsson, K., Valtonen, M.J., Kotilainen, J., Jaakkola, T.: On the redshift-apparent size diagram of double radio sources. Astrophys. J., 413, 453-476 (1993)

121. Pashchenko, I.N., Vitrishchak, V.M.: The use of ultra-compact radio sources for the angular size-redshift cosmological test. Astron. Reports, 55(4), 293-301 (2011)

122. Holanda, R.F.L., Goncalves, R.S., Alcaniz, J.S.: A test for cosmic distance duality. J. Cosmol. Astropart. Phys., 6, id. 022 (2012)

123. Totani, T., Yoshii, Y., Maihara, T., Iwamuro, F., Motohara, K.: Near-Infrared Faint Galaxies in the Subaru Deep Field: Comparing the Theory with Observations for Galaxy Counts, Colors, and Size Distributions to $K \sim 24.5$. Astrophys. J., 559, 592-605 (2001)

124. López-Corredoira, M.: Alcock-Paczyński cosmological test. Astrophys. J., 781, id. 96 , 15 pp. (2014) 
125. Melia, F., López-Corredoira, M.: Alcock-Paczyński cosmological test with modelindependent BAO data. Int. J. Mod. Phys. D, 26, id. 1750055, 10 pp. (2017)

126. Arp, H.C.: QSOs, Redshifts and Controversies. Interstellar Media, Berkeley (1987)

127. Arp, H.C. Catalogue of discordant redshift associations. Apeiron, Montreal (2003)

128. Burbidge, G.R.: Noncosmological Redshifts. Publ. Astron. Soc. Pacific, 113, 899-902 (2001)

129. Bell, M.B.: Further Evidence for Large Intrinsic Redshifts. Astrophys. J., 566, 705-711 (2002)

130. Bell, M.B.: On Quasar Distances and Lifetimes in a Local Model. Astrophys. J., 567, 801-810 (2002)

131. Bell, M.B.: Evidence that Quasars and Related Active Galaxies are Good Radio Standard Candles and that they are Likely to be a Lot Closer than their Redshifts Imply. arXiv.org: astro-ph/0602242 (2006)

132. Bell, M.B.: Further Evidence That the Redshifts of AGN Galaxies May Contain Intrinsic Components. Astrophys. J. Lett., 667, L129-L132 (2007)

133. López-Corredoira, M., Gutiérrez, C. M.: Research on candidates for non-cosmological redshifts. In: Lerner, E.J., Almeida, J.B. (eds.) 1st Crisis in Cosmology Conference (AIP Conf. Ser. 822(1)), pp. 75-92. AIP, Melville (2006)

134. López-Corredoira, M.: Apparent discordant redshift QSO-galaxy associations. In: Harutyunian, H.A., Mickaelian, A.M., Terzian, Y. (eds.) Evolution of Cosmic Objects through their Physical Activity, pp. 196-205. Gitutyun Publ. House of NAS RA, Yerevan (2010)

135. Chu, Y., Zhu, X., Burbidge, G., Hewitt, A.: Statistical evidence for possible association between QSOs and bright galaxies. Astron. Astrophys., 138, 408-414 (1984)

136. Zhu, X. F., Chu, Y. Q.: The association between quasars and the galaxies of the Virgo cluster. Astron. Astrophys., 297, 300-304 (1995)

137. Burbidge, G.R., Narlikar, J.V., Hewitt, A.: The statistical significance of close pairs of QSOs. Nature, 317, 413-415 (1985)

138. Burbidge, G.R.: The reality of anomalous redshifts in the spectra of some QSOs and its implications. Astron. Astrophys., 309, 9-22 (1996)

139. Harutyunian, H.A., Nikogossian, E.H.: Quasars in Regions of Rich Clusters of Galaxies. Astrophysics (English translation of Astrofizika), 43(4), 391-402 (2000)

140. Benítez, N., Sanz, J.L., Martínez-González, E.: Quasar-galaxy associations revisited. Mon. Not. R. Astron. Soc., 320, 241-248 (2001)

141. Gaztañaga, E.: Correlation between Galaxies and Quasi-stellar Objects in the Sloan Digital Sky Survey: A Signal from Gravitational Lensing Magnification? Astrophys. J., 589, 82-99 (2003)

142. Nollenberg, J.G., Williams, L.R.: Galaxy-Quasar Correlations between APM Galaxies and Hamburg-ESO QSOs. Astrophys. J., 634, 793-805 (2005)

143. Bukhmastova, Yu.L.: Quasars lensed by globular clusters of spiral and elliptical galaxies. Astron. Lett., 33(6), 355-367 (2007). Translated from original Russian: Pi'sma v Astronomicheckii Zhurnal, 33(6), 403 (2007)

144. Burbidge, G., Napier, W.M.: Associations of high-redshift quasi-stellar objects with active, low-redshift spiral galaxies. Astrophys. J., 706, 657-664 (2009)

145. López-Corredoira, M.: Pending Problems in QSOs. Int. J. Astron. Astrophys., 1(2), 73-82 (2011)

146. Taganov, I.N.: Quantum cosmology: deceleration of time. TIN, St.-Petersburg (2008)

147. Scranton, R., Ménard, B., Richards, G.T., et al.: Detection of Cosmic Magnification with the Sloan Digital Sky Survey. Astrophys. J., 633, 589-602

148. Primack, J.R.: Precision cosmology. New Astr. Rev., 49, 25-34 (2005)

149. Gamow, G.: The expanding universe and the origin of galaxies. Kgl. Danske Videnskab Selskab Mat. Fys. Medd., 27(10), 3-15 (1953)

150. Alpher, R.A., Herman, R.: Remarks on the Evolution of the Expanding Universe. Phys. Rev., 75, 1089-1095 (1949)

151. Novikov, I.: Discovery of CMB, Sakharov Oscillations and Polarization of the CMB Anisotropy. In: Martínez, V.J., Trimble, V., Pons-Bordería, M.J. (eds.) Historical Development of Modern Cosmology (ASP Conf. Ser. 252), pp. 43-53. Astronomical Society of the Pacific, S. Francisco (2001) 
152. Doroshkevich, A.G., Novikov, I.D.: Mean Density of Radiation in the Metagalaxy and Certain Problems in Relativistic Cosmology. Soviet Physics-Dokl., 9, 111-113 (1964). Translated from original Russian: Dokl. Akad. Nauk. USSR, 154, 809-811 (1964)

153. Van Flandern, T.: Is the microwave radiation really from the big bang 'fireball'? Reflector (Astron. League Newsletter), XLV, 4 (1993)

154. Dicke, R.H., Peebles, P.J.E., Noll, P.G., Wilkinson, D.T.: Cosmic Black-Body Radiation. Astrophys. J., 142, 414-419 (1965)

155. Mather, J.C., Cheng, E.S., Cottingham, D.A., et al.: Measurement of the cosmic microwave background spectrum by the COBE FIRAS instrument. Astrophys. J., 420, 439$444(1994)$

156. Shmaonov, T.: Pribori i Tekhnika Experimenta (Russia), 1, 83 (1957)

157. Herzberg, G.: Spectra of Diatomic Molecules. Van Nostrand, New York (1950)

158. Assis, A.K.T., Neves, M.C.D.: History of the $2.7 \mathrm{~K}$ temperature prior to Penzias and Wilson. Apeiron, 2, 79-84 (1995)

159. Meyers, R.: A Brief history of competing ideologies in cosmology and evidence for noncosmological redshifts as a case for alternative theoretical interpretations in cosmology (PhD thesis). University of Western Sydney, Sydney (2003)

160. Eddington, A.S.: Internal constitution of the stars. Cambridge University Press, Cambridge (1926, reprinted: 1988)

161. Regener, E.: Der Energiestrom der Ultrastrahlung. Zeit. Phys., 80, 666-669 (1933)

162. Nernst, W.: Weitere prüfung der annahme lines stationären zustandes im weltall. Zeit. Phys., 106, 633-661 (1937)

163. Finley-Freundlich, E.: Red shifts in the spectra of celestial bodies. Phil. Mag., 45, 303-319 (1954)

164. Born, M.: On the Interpretation of Freundlich's Red-Shift Formula. Proc. Phil. Soc., A67, 193-194 (1954)

165. Peebles, P.J.E.: The Standard Cosmological Model. In: Greco, M. (ed.) Le Rencontres de Physique de la Vallee d'Aoste: Results and Perspectives in Particle Physics, p. 39. Poligrafica Laziale s.r.l., Frascati (1998)

166. Bondi, H., Gold, T., Hoyle, F.: Black giant stars. Observatory Mag., 75, 80-81 (1955)

167. Burbidge, G.R.: Nuclear Energy Generation and Dissipation in Galaxies. Publ. Astron. Soc. Pacific, 70, 83-89 (1958)

168. Burbidge, G.R.: Explosive Cosmogony and the Quasi-Steady State Cosmology. In: Sato, K. (ed.) Cosmological Parameters and the Evolution of the Universe, pp. 286-289. Kluwer, Dordrecht (1999)

169. Hoyle, F., Wickramashinghe, N.C., Reddish, V.C.: Solid Hydrogen and the Microwave Background. Nature, 218, 1124-1126 (1968)

170. Hoyle, F., Burbidge, G., Narlikar, J.V.: A quasi-steady state cosmological model with creation of matter. Astrophys. J., 410, 437-457 (1993)

171. Hoyle, F., Burbidge, G., Narlikar, J.V.: Astrophysical Deductions from the Quasi Steadystate Cosmology. Mon. Not. R. Astron. Soc., 267, 1007-1019 (1994). Erratum: Mon. Not. R. Astron. Soc., 269, 1152 (1994)

172. Soberman, R.K., Dubin, M.: Dark Matter is Baryons. arXiv.org :astro-ph/0107550 (2001)

173. Alfonso-Faus, A., Fullana i Alfonso, M.J.F.: Sources of cosmic microwave radiation and dark matter identified: millimeter black holes (m.b.h.). arXiv.org: 1004.2251 (2010)

174. Clube, S.V.M.: The material vacuum. Mon. Not. R. Astron. Soc., 193, 385-397 (1980)

175. Sorrell, W.H. The cosmic microwave background radiation in a non-expanding universe. Astrophys. Space Sci., 317, 59 (2008)

176. Lorentz, H.A.: Electromagnetic phenomena in a system moving with any velocity less than that of light. Proc. R. Acad. Amsterdam, 6, 809-830 (1904)

177. Lerner, E.J.: Plasma model of microwave background and primordial elements - an alternative to the big bang. Laser and Particle Beams, 6, 457-469 (1988)

178. Lerner, E.J.: Intergalactic Radio Absorption and the COBE Data. Astrophys. Space Sci., 227, 61-81 (1995)

179. Lerner, E.J.: Radio absorption by the intergalactic medium. Astrophys. J., 361, 63-68 (1990)

180. Lerner, E.J.: Confirmation of radio absorption by the intergalactic medium. Astrophys. Space Sci., 207, 17-26 (1993) 
181. Garrett, M.A.: The FIR/Radio correlation of high redshift galaxies in the region of the HDF-N. Astron. Astrophys., 384, L19-L22 (2002)

182. Mao, M.Y., Huynh, M.T., Norris, R.P., Dickinson, M., Frayer, M., Helou, G., Monkiewick, J.A.: No Evidence for Evolution in the Far-infrared-Radio Correlation out to z 2 in the Extended Chandra Deep Field South. Astrophys. J., 731, id. 79, 10 pp. (2011)

183. Shpenkov, G.P., Kreidik, G.: Microwave background radiation of hydrogen atoms. Revista Ciencias Exatas e Naturais, 4,9-18 (2002)

184. Krishan, V.: Optical Depth of the Cosmic Microwave Background due to Scattering and Absorption. arXiv.org: 0909.0125 (2009)

185. Crawford, D.: Observational Evidence Favors a Static Universe (Part II). J. Cosmology, 13, 3947-3999 (2011)

186. Pecker, J.-C., Narlikar, J.V., Ochsenbein, F., Wickramasinghe, C.: The local contribution to the microwave background radiation. Res. Astron. Astrophys., 15, id. 461 (2015)

187. Planck Collaboration: Planck early results. X. Statistical analysis of Sunyaev-Zeldovich scaling relations for X-ray galaxy clusters. Astron. Astrophys., 536, id. A10, 14 pp. (2011)

188. Navia, C.E., Augusto, C.R.A., Tsui, K.H.: On the ultra high energy cosmic rays and the origin of the cosmic microwave background radiation. arXiv.org: 0707.1896 (2007)

189. Greisen, K.: End to the Cosmic-Ray Spectrum? Phys. Rev. Lett. 16, 748-750 (1966)

190. Zapsepin, G.T., Kuzmin, V.A.: Upper limit of the spectrum of cosmic rays. Sov. Phys.JETP Lett, 4, 78-80 (1966)

191. Abraham, R.G., Nair, P., McCarthy, P.J., et al.: The Gemini Deep Deep Survey. VIII. When Did Early-Type Galaxies Form? Astrophys. J., 669, 184-201 (2007)

192. Kashti, T., Waxman, E.: Searching for a correlation between cosmic-ray sources above $10^{19} \mathrm{eV}$ and large scale structure. J. Cosmol. Astropart. Phys., 5, id. 6, 21 pp. (2008)

193. López-Corredoira, M., Sylos-Labini, F., Betancort-Rijo, J.: Absence of significant crosscorrelation between WMAP and SDSS. Astron. Astrophys., 513, id. A3, 5 pp. (2010)

194. Fahr, H.J., Zönnchen, J.H.: The "writing on the cosmic wall": Is there a straightforward explanation of the cosmic microwave background? Ann. Phys., 521, 699-721 (2009)

195. Li., T.-P., Liu, H., Song, L.-M., Xiong, S.-L., Nie, J.-Y.: Observation number correlation in WMAP data. Mon. Not. R. Astron. Soc., 398, 47-52 (2009)

196. Roukema, B. F.: On the suspected timing error in Wilkinson microwave anisotropy probe map-making. Astron. Astrophys., 518, A34, 7pp. (2010)

197. Liu, H., Xiong, S.-L., Li, T.-P.: Diagnosing timing error in WMAP data. Mon. Not. R. Astron. Soc., 413, L96-L100 (2011)

198. Cover, K.S.: Sky maps without anisotropies in the cosmic microwave background are a better fit to WMAP's uncalibrated time ordered data than the official sky maps. Europhys. Lett., 87, id. 69003 (2009)

199. Sachs, R.K., Wolfe, A.M.: Perturbations of a Cosmological Model and Angular Variations of the Microwave Background. Astrophys. J., 147, 73-90 (1967)

200. de Bernardis, P., Ade, P.A.R., Bock, J.J., et al.: A flat Universe from high-resolution maps of the cosmic microwave background radiation. Nature, 404, 955-959 (2000)

201. Hanany, S., Ade, P., Balbi, A., et al.: MAXIMA-1: A Measurement of the Cosmic Microwave Background Anisotropy on Angular Scales of 10'-5 $5^{\circ}$ Astrophys. J. Lett., 545, L5-L9 (2000)

202. Hu, W., Dodelson, S.: Cosmic Microwave Background Anisotropies. Ann. Rev. Astron. Astrophys., 40, 171-216 (2002)

203. Peebles, P.J.E., Yu, J.T.: Primeval Adiabatic Perturbation in an Expanding Universe. Astrophys. J., 162, 815-836 (1970)

204. White, M., Viana, P.T.P., Liddle, A.R., Scott, D.: Primeval Adiabatic Perturbation in an Expanding Universe. Mon. Not. R. Astron. Soc., 283, 107-118 (2006)

205. Bond, J.R., Efstathiou, G.: The statistics of cosmic background radiation fluctuations. Mon. Not. R. Astron. Soc., 226, 655-687 (1987)

206. Jorgensen, H.E., Kotok, E., Naselsky, P., Novikov, I.: Evidence for Sakharov oscillations of initial perturbations in the anisotropy of the cosmic microwave background. Astron. Astrophys., 294, 639-647 (1995)

207. Hu, W., Fukugita, M., Zaldarriaga, M., Tegmark, M.: Cosmic Microwave Background Observables and Their Cosmological Implications. Astrophys. J., 549, 669-680 (2001)

208. Blanchard, A., Douspis, M., Rowan-Robinson, M., Sarkar, S.: An alternative to the cosmological "concordance model". Astron. Astrophys., 412, 35-44 (2003) 
209. Larson, D., Dunkley, J., Hinshaw, G., et al.: Seven-year Wilkinson Microwave Anisotropy Probe (WMAP) Observations: Power Spectra and WMAP-derived Parameters. Astrophys. J. Suppl. Ser., 192, id. 16, 19 pp. (2011)

210. Peiris, H.: First year Wilkinson Microwave Anisotropy Probe results: implications for cosmology and inflation. Contemporary Phys., 46(2), 77-91 (2005)

211. Disney, M.J.: Modern Cosmology: Science or Folktale? American Scientist, 95(5), 383385 (2007)

212. Jefferys, H., Berger, J.: Ockham's Razor and Bayesian Analysis. American Scientist, 80(1), 64-72 (1992)

213. Berger, J.O., Jefferys, W.H.: The application of robust Bayesian analysis to hypothesis testing and Occam's Razor. J. Italian Statistical Soc., 1, 17-32 (1992)

214. Soler Gil, F.J.: Modelos cosmológicos: ¿Ficciones útiles o descripciones realistas del universo? Thémata, 40, 117-146 (2008)

215. López-Corredoira, M., Gabrielli, A.: Peaks in the CMBR power spectrum. I. Mathematical analysis of the associated real space features. Physica A, 392, 474-484 (2013)

216. López-Corredoira, M.: Peaks in the CMBR Power Spectrum II: Physical Interpretation for any Cosmological Scenario. Int. J. Mod. Phys. D, 22(7), id. 1350032 (2013)

217. Narlikar, J.V., Vishwakarma, R.G., Hajian, A., Souradeep, T., Burbidge, G.; Hoyle, F.: Inhomogeneities in the Microwave Background Radiation Interpreted within the Framework of the Quasi-Steady State Cosmology. Astrophys. J., 585, 1-11 (2003)

218. Narlikar, J.V., Burbidge, G., Vishwakarma, R.G.: Cosmology and cosmogony in a cyclic universe. J. Astrophys. Astron., 28, 67-99 (2007)

219. Walker, M., Ohishi, M., Mori, M.: Microwave anisotropies from the Galactic halo. arXiv.org: astro-ph/0210483 (2002)

220. McGaugh, S.S.: Confrontation of Modified Newtonian Dynamics Predictions with Wilkinson Microwave Anisotropy Probe First Year Data. Astrophys. J., 611, 26-39 (2004) 26

221. Angus, G.W., Diaferio, A.: The abundance of galaxy clusters in modified Newtonian dynamics: cosmological simulations with massive neutrinos. Mon. Not. R. Astron. Soc., 417, 941-949 (2011)

222. Dodelson, S.: Coherent Phase Argument for Inflation. In: Nieves, J.F., Raymond, R. (eds.) Neutrinos, Flavor Physics and Precision Cosmology (AIP Conf. Proc. 689), pp. 184-196. AIP, Melville (New York) (2003)

223. Coulson, D., Ferreira, P., Graham, P., Turok, N.: Microwave anisotropies from cosmic defects. Nature, 368, 27-31 (1994)

224. López-Corredoira, M.: Some doubts on the validity of the foreground Galactic contribution subtraction from microwave anisotropies. J. Astrophys. Astron., 28, 101-116 (2007)

225. Ferreira, P.G., Magueijo, J., Górski, K.M.: Evidence for Non-Gaussianity in the COBE DMR 4 Year Sky Maps. Astrophys. J. Lett., 503, L1-L4 (1998)

226. Pando, J., Valls-Gabaud, D., Fang, L.-Z.: Evidence for Scale-Scale Correlations in the Cosmic Microwave Background Radiation. Phys. Rev. Lett., 81(21), 4568-4571 (1998)

227. Jeong, E., Smoot, G.F.: Probing Non-Gaussianity In The Cosmic Microwave Background Anisotropies: One Point Distribution Function. arXiv.org: 0710.2371 (2007)

228. Raeth, C., Schuecker, P., Banday, A.J.: A scaling index analysis of the Wilkinson Microwave Anisotropy Probe three-year data: signatures of non-Gaussianities and asymmetries in the cosmic microwave background. Mon. Not. R. Astron. Soc., 380, 466-478 (2007)

229. Bernui, A., Tsallis, C., Villela, T.: Deviation from Gaussianity in the cosmic microwave background temperature fluctuations. Europhys. Lett., 78(1), id. 19001 (2007)

230. McEwen, J.D., Hobson, M.P., Lasenby, A.N., Mortlock, D.J.: A high-significance detection of non-Gaussianity in the WMAP 5-yr data using directional spherical wavelets. Mon. Not. R. Astron. Soc., 388, 659-662 (2008)

231. Rossi, G., Sheth, R. K., Park, C., Hernández-Monteagudo, C.: Non-Gaussian distribution and clustering of hot and cold pixels in the five-year WMAP sky. Mon. Not. R. Astron. Soc., 399, 304-316 (2009)

232. Rubiño-Martín, J.A., Aliaga, A.M., Barreiro, R.B., et al.: Non-Gaussianity in the Very Small Array cosmic microwave background maps with smooth goodness-of-fit tests. Mon. Not. R. Astron. Soc., 369, 909-920 (2006) 
233. McEwen, J.D., Hobson, M.P., Lasenby, A.N., Mortlock, D.J.: A high-significance detection of non-Gaussianity in the WMAP 3-yr data using directional spherical wavelets. Mon. Not. R. Astron. Soc., 371, L50-L54 (2006)

234. Liu, X., Zhang, S.N.: Non-Gaussianity Due to Possible Residual Foreground Signals in Wilkinson Microwave Anistropy Probe First-Year Data Using Spherical Wavelet Approaches. Astrophys. J., 633, 542-551 (2005)

235. Tojeiro, R., Castro, P.G., Heavens, A.F., Gupta, S.: Non-Gaussianity in the Wilkinson Microwave Anisotropy Probe data using the peak-peak correlation function. Mon. Not. R. Astron. Soc., 365, 265-275 (2006)

236. Chiang, L.-Y., Naselsky, P.D., Coles, P.: Departure from Gaussianity of the Cosmic Microwave Background Temperature Anisotropies in the Three-Year WMAP Data. Astrophys. J., 664, 8-13 (2007)

237. Gutierrez de La Cruz, C.M., Davies, R.D., Rebolo, R., Watson, R.A., Hancock, S., Lasenby, A.N.: Dual-frequency mapping with the Tenerife cosmic microwave background experiments. Mon. Not. R. Astron. Soc., 442, 10-22 (1995)

238. Davies, R.D., Gutiérrez, C.M., Hopkins, J., et al.: Studies of cosmic microwave background structure at Dec. $=+40 \mathrm{deg}-\mathrm{I}$. The performance of the Tenerife experiments. Mon. Not. R. Astron. Soc., 278, 883-896 (1996)

239. Bennett, C.L., Hill, R.S., Hinshaw, G., et al.: First-Year Wilkinson Microwave Anisotropy Probe (WMAP) Observations: Foreground Emission. Astrophys. J. Supp. Ser., 148, 97-117 (2003)

240. Leitch, E.M., Readhead, A.C.S., Pearson, T.J., Myers, S.T., Gulkis, S., Lawrence, C.R.: A Measurement of Anisotropy in the Cosmic Microwave Background on 7'-22' Scales. Astrophys. J., 532, 37-56 (2000)

241. Kogut A., Banday A.J., Bennett C.L., et al.: High-Latitude Galactic Emission in the COBE Differential Microwave Radiometer 2 Year Sky Maps. Astrophys. J., 460, 1-9 (1996)

242. Finkbeiner, D.P., Langston, G.I., Minter, A.H.: Microwave Interstellar Medium Emission in the Green Bank Galactic Plane Survey: Evidence for Spinning Dust. Astrophys. J., 617, 350-359 (2004)

243. Fernández-Cerezo, S., Gutiérrez, C.M., Rebolo, R., et al.: Observations of the cosmic microwave background and galactic foregrounds at $12-17 \mathrm{GHz}$ with the COSMOSOMAS experiment. Mon. Not. R. Astron. Soc., 370, 15 (2006)

244. Casassus, S., Readhead, A.C.S., Pearson, T.J., Nyman, L.-A., Shepherd, M.C., Bronfman, L.: Anomalous Radio Emission from Dust in the Helix. Astrophys. J., 603, 599-610 (2004)

245. Watson, R.A., Rebolo, R., Rubiño-Martín, J.A., Hildebrandt, S., Gutiérrez, C.M., Fernández-Cerezo, S., Hoyland, R.J., Battistelli, E.S.: Detection of Anomalous Microwave Emission in the Perseus Molecular Cloud with the COSMOSOMAS Experiment. Astrophys. J. Lett., 624, L89-L92 (2005)

246. de Oliveira-Costa A., Tegmark, M., Gutiérrez, C.M., Jones, A.W., Davies, R.D., Lasenby, A.N., Rebolo, R., Watson, R.A.: Cross-Correlation of Tenerife Data with Galactic Templates-Evidence for Spinning Dust? Astrophys. J. Lett., 527, L9-L12 (1999)

247. de Oliveira-Costa, A., Tegmark, M., Finkbeiner, D.P., et al.: A New Spin on Galactic Dust. Astrophys. J., 567, 363-369 (2002)

248. Draine B.T., Lazarian A.: Diffuse Galactic Emission from Spinning Dust Grains. Astrophys. J. Lett., 494, L19-L22 (1998)

249. Draine B.T., Lazarian A.: Magnetic Dipole Microwave Emission from Dust Grains. Astrophys. J., 512, 740-754 (1999)

250. Pietrobon, D., Górski, K.M., Bartlett, J., et al.: Analysis of WMAP 7 Year Temperature Data: Astrophysics of the Galactic Haze. Astrophys. J., 755, id. 69, 16 pp. (2012)

251. Finkbeiner, D.P., Davis, M., Schlegel, D.J.: Extrapolation of Galactic Dust Emission at 100 Microns to Cosmic Microwave Background Radiation Frequencies Using FIRAS. Astrophys. J., 524, 867-886 (1999)

252. López-Corredoira, M.: A conspicuous increase of Galactic contamination over CMBR anisotropies at large angular scales. Astron. Astrophys., 346, 369-382 (1999)

253. Bottino, M., Banday, A.J., Maino, D.: Foreground analysis of the Wilkinson Microwave Anisotropy Probe 3-yr data with FASTICA. Mon. Not. R. Astron. Soc., 389, 1190-1208 (2008) 
254. Schlegel, D.J., Finkbeiner, D.P., Davis, M.: Maps of Dust Infrared Emission for Use in Estimation of Reddening and Cosmic Microwave Background Radiation Foregrounds. Astrophys. J., 500, 525-553 (1998)

255. Planck Collaboration: Planck 2015 results. I. Overview of products and scientific results. Astron. Astrophys., 594, id. A1, 38 pp. (2016)

256. Axelsson, M., Ihle, H.T., Scodeller, S., Hansen, F.K. Testing for foreground residuals in the Planck foreground cleaned maps: A new method for designing confidence masks. Astron. Astrophys., 578, id. A44, 11 pp. (2015)

257. Masi, S., Ade, P.A.R., Bock, J.J., et al.: High-Latitude Galactic Dust Emission in the BOOMERANG Maps. Astrophys. J. Lett., 553, L93-L96 (2001)

258. Kiss, C., Ábrahám, P., Klaas, U., Lemke, D., Héraudeau, P., del Burgo, C., Herbstmeier, U.: Small-scale structure of the galactic cirrus emission. Astron. Astrophys., 399, 177-185 (2003)

259. Planck Collaboration: Planck early results. XXIII. The first all-sky survey of Galactic cold clumps. Astron. Astrophys., 536, id. A23, 33 pp. (2011)

260. Tegmark, M.: Removing Real-World Foregrounds from Cosmic Microwave Background Maps. Astrophys. J., 502, 1-6 (1998)

261. Robitaille, P.-M.: WMAP: A Radiological Analysis. Progress in Physics, 1/2007, 3-18 (2007)

262. Eriksen, H.K., Banday, A.J., Górski, K.M., Lilje, P.B.: On Foreground Removal from the Wilkinson Microwave Anisotropy Probe Data by an Internal Linear Combination Method: Limitations and Implications. Astrophys. J., 612, 633-646 (2004)

263. Eriksen, H.K., Banday, A.J., Górski, K.M., Lilje, P.B.: Astro-ph communication: Simulations of the WMAP Internal Linear Combination sky map. arXiv.org: astro-ph/0508196 (2005)

264. Vio, R., Andreani, P.: A statistical analysis of the "internal linear combination" method in problems of signal separation as in cosmic microwave background observations. Astron. Astrophys., 487, 775-780 (2008)

265. Hansen, F.K., Banday, A.J., Eriksen, H.K., Górski, K.M., Lilje, P.B.: Foreground Subtraction of Cosmic Microwave Background Maps Using WI-FIT (Wavelet-Based HighResolution Fitting of Internal Templates). Astrophys. J., 648, 784-796 (2006)

266. Naselsky, P.D., Novikov, I.G., Chiang, L.-Y.: Correlations from Galactic Foregrounds in the First-Year Wilkinson Microwave Anisotropy Probe Data. Astrophys. J., 642, 617624 (2006)

267. de Oliveira-Costa, A., Tegmark, M.: CMB multipole measurements in the presence of foregrounds. Phys. Rev. D, 74(2), id. 023005 (2006)

268. Then, H.: Foreground contamination of the WMAP CMB maps from the perspective of the matched circle test. Mon. Not. R. Astron. Soc., 373, 139-145 (2006)

269. Samal, P.K., Saha, R., Jain, P., Ralston, J.P.: Testing isotropy of cosmic microwave background radiation. Mon. Not. R. Astron. Soc., 385, 1718-1728 (2008)

270. Liu, X., Zhang, S.N.: A Cross-Correlation Analysis of WMAP and EGRET Data in Wavelet Space. Astrophys. J. Lett., 636, L1-L4 (2006)

271. Verschuur, G.L.: High Galactic Latitude Interstellar Neutral Hydrogen Structure and Associated (WMAP) High-Frequency Continuum Emission. Astrophys. J., 671, 447-457 (2007)

272. Sarkar, S.: Does the galactic synchrotron radio background originate in old supernova remnants. Mon. Not. R. Astron. Soc., 199, 97-108 (1982)

273. Sarkar, S.: Galactic foregrounds for the CMB. Paper (PoS(FFP14)095) presented at the Frontiers of Fundamental Physics, Marseille, France, 15-18 July 2014

274. Liu, H., Mertsch, P., Sarkar, S.: Fingerprints of Galactic Loop I on the Cosmic Microwave Background. Astrophys. J. Lett., 789, id. L29, 5 pp. (2014)

275. Copi, C.J., Huterer, D., Schwarz, D.J., Starkman, G.D.: On the large-angle anomalies of the microwave sky. Mon. Not. R. Astron. Soc., 367, 79-102 (2006)

276. Copi, C.J., Huterer, D., Schwarz, D.J., Starkman, G.D.: Uncorrelated universe: Statistical anisotropy and the vanishing angular correlation function in WMAP years 1-3. Phys. Rev. D, 75(2), id. 23507 (2007)

277. Su, S.-C., Chu, M.-C.: New Anomalies in Cosmic Microwave Background Anisotropy: Violation of the Isotropic Gaussian Hypothesis in Low- $\ell$ Modes. arXiv.org: 0805.1316 (2008) 
278. Starkman, G.D., Copi, C.J., Huterer, D., Schwarz, D.: The Oddly Quiet Universe: How the CMB challenges cosmology's standard model. arXiv.org: 1201.2459 (2012)

279. Rakić, A., Schwarz, D.J.: Correlating anomalies of the microwave sky. Phys. Rev. D, 75(10), id. $103002(2007)$

280. Copi, C.J., Huterer, D., Schwarz, D.J, Starkman, G.D.: No large-angle correlations on the non-Galactic microwave sky. Mon. Not. R. Astron. Soc., 399, 295-303 (2009)

281. Liu, H., Li, T.-P.: Missing completely of CMB quadrupole in WMAP data. Chinese Science Bull., 58, 1243-1249 (2013)

282. Eriksen, H.K., Hansen, F.K., Banday, A.J., Górski, K.M., Lilje, P.B.: Asymmetries in the Cosmic Microwave Background Anisotropy Field. Astrophys. J., 605, 14-20 (2004)

283. Jiang, B.-Z., Lieu, R., Zhang, S.-N., Wakker, B.: Significant Foreground Unrelated Non-Acoustic Anisotropy on the 1 Degree Scale in Wilkinson Microwave Anisotropy Probe 5-Year Observations. Astrophys. J., 708, 375-380 (2010)

284. Bennett, C.L., Hill, R.S., Hinshaw, G., et al.: Seven-year Wilkinson Microwave Anisotropy Probe (WMAP) Observations: Are There Cosmic Microwave Background Anomalies? Astrophys. J. Supp. Ser., 192, id. 17, 19 pp. (2011)

285. Planck Collaboration: Planck 2015 results. XVI. Isotropy and statistics of the CMB. Astron. Astrophys., 594, id. A16, 62 pp. (2016)

286. Chyzy, K.T., Novosyadlyj, B., Ostrowski, M.: Gradient and dispersion analyses of the WMAP data. arXiv.org: astro-ph/0512020 (2005)

287. Abramo, L.R., Sodre, L. Jr., Wuensche, C.A.: Anomalies in the low CMB multipoles and extended foregrounds. Phys. Rev. D, 74(8), id. 83515 (2006)

288. Sharpe, H.N.: Heliosheath Synchrotron Radiation As A Possible Source For The Arcade 2 CMB Distortions. arXiv.org: 0902.0181 (2009)

289. Sharpe, H.N.: A Model For The WMAP Anomalous Ecliptic Plane Signal. arXiv.org: $0904.1697(2009)$

290. Sharpe, H.N.: A Heliosheath Model for the Origin of the CMB Quadrupole Moment. arXiv-org: 0905.2978 (2009)

291. Meliá, F.: Cosmological Implications of the CMB Large-Scale Structure. Astron. J., 149, id. 6, 10 pp. (2015)

292. Lieu, R., Mittaz, J.P.D.: On the Absence of Gravitational Lensing of the Cosmic Microwave Background. Astrophys. J., 628, 583-593 (2005)

293. Lieu, R., Mittaz, J.P.D., Zhang, S.-N.: The Sunyaev-Zel'dovich Effect in a Sample of 31 Clusters: A Comparison between the X-Ray Predicted and WMAP Observed Cosmic Microwave Background Temperature Decrement. Astrophys. J., 648, 176-199 (2006)

294. Bonamente, M., Joy, M.K., LaRoque, S.J., Carlstrom, J.E., Reese, E.D., Dawson, K. S.: Determination of the Cosmic Distance Scale from Sunyaev-Zel'dovich Effect and Chandra X-Ray Measurements of High-Redshift Galaxy Clusters. Astrophys. J., 647, 25-54 (2006)

295. Lieu, R., Quenby, J., Bonamente, M.: The Non-thermal Intracluster Medium. Astrophys. J., 721, 1482-1491 (2010)

296. Burbidge, G.R.: Was there really a Big Bang? Nature, 233, 36 (1971)

297. Burbidge, G.R., Hoyle, F.: The Origin of Helium and the Other Light Elements. Astrophys. J. Lett., 509, L1-L3 (1998)

298. Salvaterra, R., Ferrara, A.: Is primordial ${ }^{4}$ He truly from the Big Bang? Mon. Not. R. Astron. Soc., 340, L17-L20 (2003)

299. Schramm, D.N., Turner, M.S.: Big-bang nucleosynthesis enters the precision era. Rev. Mod. Phys., 70, 303-318 (1998)

300. Izotov, Y.I., Thuan, T.X.: The Primordial Abundance of ${ }^{4} \mathrm{He}$ : Evidence for NonStandard Big Bang Nucleosynthesis. Astrophys. J. Lett., 710, L67-L71 (2010)

301. Terlevich, E., Terlevich, R., Skillman, E., Stepanian, J., Lipovetskii, V.: The Extremely Low He Abundance of SBS:0335-052. In: Edmunds, M.G., Terlevich, R. (eds.) Elements and the Cosmos, pp. 21-27. Cambridge University Press, Cambridge (1992)

302. Sargent, W.L.W., Searle, L.: The Interpretation of the Helium Weakness in Halo Stars. Astrophys. J. Lett., 150, L33-L37 (1967)

303. Casagrande, L., Flynn, C., Portinari, L., Girardi, L., Jiménez, R.: The helium abundance and $\Delta Y / \Delta Z$ in lower main-sequence stars. Mon. Not. R. Astron. Soc., 382, 1516$1540(2007)$

304. Ryan, S.G., Beers, T.C., Olive, K.A., Fields, B.D., Norris, J.E.: Primordial Lithium and Big Bang Nucleosynthesis. Astrophys. J. Lett., 530, L57-L60 (2000) 
305. Coc, A., Goriley, S., Xu, Y., Saimpert, M., Vangioni, E.: Standard Big Bang Nucleosynthesis up to CNO with an Improved Extended Nuclear Network. Astrophys. J., 744, id. 158,18 pp. $(2012)$

306. Famaey, B., McGaugh, S.: Modified Newtonian Dynamics (MOND): Observational Phenomenology and Relativistic Extensions. Living Rev. Relativity, 15, id. 10, 159 pp. (2012)

307. Cyburt, R.H., Fields, B.D., Olive, K.A.: An update on the big bang nucleosynthesis prediction for ${ }^{7} \mathrm{Li}$ : the problem worsens. J. Cosmol. Astropart. Phys., 11, id. 12, 14 pp. (2008)

308. Korn, A.J., Grundahl, F., Richard, O., Barklem, P.S., Mashonkina, L., Collet, R., Piskunov, N., Gustafsson, B.: A probable stellar solution to the cosmological lithium discrepancy. Nature, 442, 657-659 (2006)

309. Howk, J.C., Lehner, N., Fields, B.D., Mathews, G.J.: Observation of interstellar lithium in the low-metallicity Small Magellanic Cloud. Nature, 489, 121-123 (2012)

310. Vidal-Madjar, A., Ferlet, R., Lemoine, M.: Deuterium Abundance and Cosmology. In: Brandt, J.C., Ake, T.B. III, Petersen, C.C. (eds.) The Scientific Impact of the Goddard High Resolution Spectrograph (ASP Conf. Series, 143), pp. 3-17. Astron. Soc of the Pacific, S. Francisco (1998)

311. Prodanovic, T., Fields, B.D.: On Nonprimordial Deuterium Production by Accelerated Particles. Astrophys. J., 597, 48-56 (2003)

312. Casuso, E., Beckman, J.E.: Beryllium and Boron Evolution in the Galaxy. Astrophys. J., 475, 155-162 (1997)

313. Boyd, R., Kajino, T.: Can Be-9 provide a test of cosmological theories? Astrophys. J. Lett., 336, L55-L58 (1989)

314. Hata, N., Scherrer, R.J., Steigman, G., Thomas, D., Walker, T.P., Bludman, S., Langacker. P.: Big Bang nucleosynthesis in crisis? Phys. Rev. Lett., 75, 3977-3980 (1995)

315. Kurucz, R.L.: Gedanken Astrophysics: The Universe Since Recombination. Comments on Astrophysics, 16, 1-15 (1992)

316. Takei, Y., Henry, J. P., Finoguenov, A., Mitsuda, K., Tamura, T., Fujimoto, R., Briel, U.G.: Warm-Hot Intergalactic Medium Associated with the Coma Cluster. Astrophys. J., 655, 831-842 (2007)

317. Anderson, M.E., Bregman, J.N.: Do Hot Halos Around Galaxies Contain the Missing Baryons? Astrophys. J., 714, 320-331 (2010)

318. McGaugh, S.S., Schombert, J.M., de Blok, W.J.G., Zagursky, M.J.: The Baryon Content of Cosmic Structures. Astrophys. J. Lett., 708, L14-L17 (2010)

319. Eckert, D., Jauzac, M., Shan H., et al.: Warmhot baryons comprise 510 per cent of filaments in the cosmic web. Nature, 528, 105-107 (2015)

320. Becker, R.H., Fan, X., White, R.L., et al.: Evidence for Reionization at $z \sim 6$ : Detection of a Gunn-Peterson Trough in a $z=6.28$ Quasar. Astron. J., 122, 2850-2857 (2001)

321. Fan, X., Narayanan, V.K., Lupton, R.H., et al.: A Survey of $z>5.8$ Quasars in the Sloan Digital Sky Survey. I. Discovery of Three New Quasars and the Spatial Density of Luminous Quasars at $z \sim 6$. Astron. J., 122, 2833-2849 (2001)

322. Malhotra, S., Rhoads, J.: Luminosity Functions of Ly $\alpha$ Emitters at Redshifts $z=6.5$ and $z=5.7$ : Evidence against Reionization at $z<=6.5$. Astrophys. J. Lett., 617, L5-L8 (2004)

323. Jarosik, N., Bennett, C. L., Dunkley, J., et al.: Seven-year Wilkinson Microwave Anisotropy Probe (WMAP) Observations: Sky Maps, Systematic Errors, and Basic Results. Astrophys. J. Supp. Ser., 192, id. 14, 15 pp. (2011)

324. Planck Collaboration. Planck 2015 results. XIII. Cosmological parameters. Astron. Astrophys., 594, id. A13, 63 pp. (2016)

325. Bunker, A.J., Wilkins, S., Ellis, R.S., et al.: The contribution of high-redshift galaxies to cosmic reionization: new results from deep WFC3 imaging of the Hubble Ultra Deep Field. Mon. Not. R. Astron. Soc., 409, 855-866 (2010)

326. Bouwens, R.J., Illingworth, G.D., Labbe, I., et al.: A candidate redshift $z \sim 10$ galaxy and rapid changes in that population at an age of 500 Myr. Nature, 469, 504-507 (2011) 327. Dopita, M.A., Krauss, L.M., Sutherland, R.S., Kobayashi, C., Lineweaver, C.H.: Reionizing the universe without stars. Astrophys. Space Sci., 335, 345-352 (2011)

328. Battaner, E., Florido, E., Jiménez-Vicente, J.: Magnetic fields and large scale structure in a hot universe. I. General equations. Astron. Astrophys., 326, 13-22 (1997) 
329. Florido, E., Battaner, E.: Magnetic fields and large-scale structure in a hot universe.

II. Magnetic flux tubes and filamentary structure. Astron. Astrophy., 327, 1-7 (1997)

330. Battaner, E., Florido, E., García-Ruiz, J. M.: Magnetic fields and large scale structure in a hot Universe. III. The polyhedric network. Astron. Astrophys., 327, 8-10 (1997)

331. Battaner, E., Florido, E.: Magnetic fields and large scale structure in a hot Universe. IV. The egg-carton Universe. Astron. Astrophys., 338, 383-385 (1998)

332. Nayeri, A., Engineer, S., Narlikar, J.V., Hoyle, F.: Structure Formation in the QuasiSteady State Cosmology: A Toy Model. Astrophys. J., 525, 10-16 (1999)

333. Broadhurst, T.J., Ellis, R.S., Koo, D.C., Szalay, A.S.: Large-scale distribution of galaxies at the Galactic poles Nature, 343, 726-728 (1990)

334. Kurki-Suonio, H.: Galactic beads on a cosmic string. Sci. News, 137, 287 (1990)

335. Kaiser, N., Peacock, J.A.: Power-spectrum analysis of one-dimensional redshift surveys. Astrophys. J., 379, 482-506 (1991)

336. Einasto, J., Einasto, M., Gottlöber, S., et al.: A 120-Mpc periodicity in the threedimensional distribution of galaxy superclusters. Nature, 385, 139-141 (1997)

337. Nabokov, N.V., Baryshev, Yu.V.: Method for analyzing the spatial distribution of galaxies on gigaparsec scales. II. Application to a grid of the HUDF-FDF-COSMOS-HDF surveys. Astrophysics, 53(1), 101-111 (2010). Translated from Russian: Astrofizika, 53(1), $117-129$ (2010)

338. Massey, R., Rhodes, J., Ellis, R., et al.: Dark matter maps reveal cosmic scaffolding. Nature, 445, 286-290 (2007)

339. Haggerty, M.J., Wertz, J.R.: On the redshift-magnituderelation in hierarchical cosmologies. Mon. Not. R. Astron. Soc., 155, 495-503 (1972)

340. Fang, L.L., Mo, H.J., Ruffini, R.: The cellular structure of the universe and cosmological tests. Astron. Astrophys., 243, 283-294 (1991)

341. Ribeiro, M.B.: On modeling a relativistic hierarchical (fractal) cosmology by Tolman's spacetime. II - Analysis of the Einstein-de Sitter model. Astrophys. J., 395, 29-33 (1992)

342. Ribeiro, M.B.: On modeling a relativistic hierarchical (fractal) cosmology by Tolman's spacetime. I - Theory. Astrophys. J., 388, 1-8 (1992)

343. Ribeiro, M.B.: On Modeling a Relativistic Hierarchical (Fractal) Cosmology by Tolman's Spacetime. III. Numerical Results. Astrophys. J, 415, 469-485 (1993)

344. Best, J.S.: An Examination of the Large-Scale Clustering of the Las Campanas Redshift Survey. Astrophys. J., 541, 519-526 (2000)

345. de Lapparent, V., Geller, M.J., Huchra, J.P.: A slice of the universe. Astrophys. J. Lett., 302, L1-L5 (1986)

346. Dressler, A., Faber, S.M., Burstein, D., Davies, R.L., Lynden-Bell, D., Terlevich, R.J., Wegner, G.: Spectroscopy and photometry of elliptical galaxies - A large-scale streaming motion in the local universe. Astrophys. J. Lett., 313, L37-L42 (1987)

347. Sandage, A.

The redshift-distance relation. IX - Perturbation of the very nearby velocity field by the mass of the Local Group. Astrophys. J., 307, 1-19 (1986)

348. Ekholm, T., Baryshev, Yu., Teerikorpi, P., Hanski, M.O., Paturel, G.: On the quiescence of the Hubble flow in the vicinity of the Local Group. A study using galaxies with distances from the Cepheid PL-relation. Astron. Astrophys., 368, L17-L20 (2001)

349. Karachentsev, I.D., Sharina, M.E., Makarov, D.I., et al.: The very local Hubble flow. Astron. Astrophys., 389, 812-824 (2002)

350. Matravers, D.R., Ellis, G.F.R., Stoeger, W.R.: Complementary Approaches to Cosmology - Relating Theory and Observations. Q. J. R. astr. Soc., 36, 29-45 (1995)

351. Lauer, T.R., Postman, M.: The motion of the Local Group with respect to the 15,000 kilometer per second Abell cluster inertial frame. Astrophys. J., 425, 418-438 (1994)

352. Mathewson, D.S., Ford, V.L., Buchhorn, M.: No back-side infall into the Great Attractor. Astrophys. J. Lett., 389, L5-L8 (1992)

353. Lindley, D.: Not so Great Attractor? Nature, 356, 657 (1992)

354. Finkbeiner, A.: Mapping the River in the Sky. Science, 257, 1208-1210 (1992)

355. Hudson, M.J., Smith, R.J., Lucey, J.R., Schelegel, D.J., Davies, R.L.: A Large-scale Bulk Flow of Galaxy Clusters. Astrophys. J. Lett., 512, L79-L82 (1999)

356. Lee, J., Komatsu, E.: Bullet Cluster: A Challenge to $\Lambda$ CDM Cosmology. Astrophys.

J., 718, 60-65 (2010) 
357. Thompson, R., Nagamine, K.: Pairwise velocities of dark matter haloes: a test for the cold dark matter model using the bullet cluster. Mon. Not. R. Astron. Soc., 419, 3560-3570 (2012)

358. Ayaita, Y., Weber, M., Wetterich, C.: Peculiar Velocity Anomaly from Forces Beyond Gravity? arXiv.org: 0908.2903 (2009)

359. Kashlinsky, A., Atrio-Barandela, F., Kocevski, D., Ebeling, H.: A measurement of large-scale peculiar velocities of clusters of galaxies: results and cosmological implications. Astrophys. J. Lett., 686, L49-L52 (2009)

360. Atrio-Barandela, F., Kashlinsky, A., Ebeling, H., Fixsen, D.J., Kocevski, D.: Probing the Dark Flow Signal in WMAP 9 -Year and Planck Cosmic Microwave Background Maps. Astrophys. J., 810, id. 143, 16 pp. (2015)

361. Tikhonov, A.V., Gottlöber, S., Yepes, G., Hoffman, Y.: The sizes of minivoids in the local Universe: an argument in favour of a warm dark matter model? Mon. Not. R. Astron. Soc., 399, 1611-1621 (2009)

362. Peebles, P.J.E., Nusser, A.: Nearby galaxies as pointers to a better theory of cosmic evolution. Nature, 465, 565-569 (2010)

363. Perivolaropoulos, L.: Six Puzzles for LCDM Cosmology. arXiv.org: 0811.4684 (2008)

364. Anderson, L., Aubourg, E., Bailey, S., et al.: The clustering of galaxies in the SDSS-III Baryon Oscillation Spectroscopic Survey: baryon acoustic oscillations in the Data Releases 10 and 11 Galaxy samples. Mon. Not. R. Astron. Soc., 441, 24-62 (2014)

365. Roukema, B.F., Buchert, T., Ostrowski, J.J., France, M.J.: Evidence for an environment-dependent shift in the baryon acoustic oscillation peak. Mon. Not. R. Astron. Soc., 448, 1660-1673 (2015)

366. Tifft, W.G.: Discrete states of redshift and galaxy dynamics. I - Internal motions in single galaxies. Astrophys. J., 206, 38-56 (1976)

367. Tifft, W.G.: Discrete states of redshift and galaxy dynamics. II - Systems of galaxies. Astrophys. J., 211, 31-46 (1977)

368. Tifft, W.G.: Periodicity in the redshift intervals for double galaxies. Astrophys. J., 236, 70-74 (1980)

369. Guthrie, B., Napier, W.M.: Redshift periodicity in the Local Supercluster. Astron. Astrophys., 310, 353-370 (1996)

370. Napier, W.M.: Statistics of redshift periodicities. In: Pecker, J.-C., Narlikar, J. (eds.) Current issues in cosmology, pp. 207-216. Cambridge University Press, Cambridge (U.K.) (2006)

371. Burbidge, G.R., O'Dell, S.L.: The Distribution of Redshifts of Quasi-Stellar Objects and Related Emission-Line Objects. Astrophys. J., 178, 583-606 (1972)

372. Bell, M.B.: Discrete Intrinsic Redshifts from Quasars to Normal Galaxies. arXiv.org: astro-ph/0211091 (2002)

373. Bell, M.B., Comeau, S.P.: Further Evidence for Quantized Intrinsic Redshifts in Galaxies: Is the Great Attractor a Myth? arXiv.org: astro-ph/0305112 (2003)

374. Bell, M.B., Comeau, S.P.: Intrinsic Redshifts in QSOs Near NGC 6212. arXiv.org: astro-ph/0306042 (2003)

375. Bell, M.B., McDiarmid, D.: Six Peaks Visible in the Redshift Distribution of 46,400 SDSS Quasars Agree with the Preferred Redshifts Predicted by the Decreasing Intrinsic Redshift Model. Astrophys. J., 648, 140-147 (2006)

376. Hartnett, J.G., Hirano, K. Galaxy redshift abundance periodicity from Fourier analysis of number counts $\mathrm{N}(\mathrm{z})$ using SDSS and 2dF GRS galaxy surveys. Astrophys. Space Sci. 328, 13-24 (2008)

377. Hartnett, J.G.: Unknown selection effect simulates redshift periodicity in quasar number counts from Sloan Digital Sky Survey Astrophys. Space Sci., 324, 13-16 (2009)

378. Fulton, C.C., Arp, H.C.: The 2dF Redshift Survey. I. Physical Association and Periodicity in Quasar Families. Astrophys. J., 754, id. 134, 10 pp. (2012)

379. Hawkins, E., Maddox, S., Merrifield, M.: No periodicities in 2dF Redshift Survey data Mon. Not. R. Astron. Soc. 336, L13-L16 (2002)

380. Tang, S.M., Zhang, S.N.: Critical Examinations of QSO Redshift Periodicities and Associations with Galaxies in Sloan Digital Sky Survey Data. Astrophys. J., 633, 41-51 (2005) 
381. Tang, S., Zhang, S.N.: Evidence against non-cosmological redshifts of QSOs in SDSS data. In: Basu, D. (ed.) Redshifts in Spectral Lines of Quasi Stellar Objects, pp. 125-136. Research Signpost, Kerala (2010)

382. Bajan, K., Flin, P., Godlowski, W., Pervushin, V.P.: On the investigations of galaxy redshift periodicity. Physics of Particles and Nuclei Letters, 4(1), 5-10 (2007)

383. Repin, S.V., Komberg, B.V., Lukash, V.N.: Absence of a periodic component in the quasar z distribution. Astron. Reports, 56(9), 702-709 (2012)

384. Hirano, K., Komiya, Z.: Observational tests for oscillating expansion rate of the Universe. Phys. Rev. D, 82(10), id. 103513 (2010)

385. Lehto, A.: Periodic time and the stationary properties of matter. Chin. J. Phys., 28, 215-225 (1990)

386. Lehto, A.: On the Planck scale and properties of matter. Nonlinear Dyn., 55, 279-298 (2009)

387. Taubes, G.: Theorists Nix Distant Antimatter Galaxies. Science, 278, 226 (1997)

388. Steinhardt, P.J., La inflación a debate. Investigación y Ciencia, Junio 2011, 16-23 (2011)

389. Martin, J., Ringeval, C., Vennin, V.: Encyclopaedia Inflationaris. Physics of the Dark Universe, 5, 75-235 (2014)

390. Zwicky, F.: Die Rotverschiebung von extragalaktischen Nebeln Helvetica Phys. Acta, 6(10), 110-127 (1933)

391. Kahn F.D., Woltjer L.: Intergalactic Matter and the Galaxy. Astrophys. J., 130, 705$717(1959)$

392. Page, T.: Radial Velocities and Masses of Double Galaxies. Astrophys. J., 116, 63-84 (1952)

393. Page, T.: Average Masses and Mass-Luminosity Ratios of the Double Galaxies. Astrophys. J., 132, 910-912 (1960)

394. Holmberg, E.: On the masses of double galaxies. Meddelanden fran Lunds Astronomiska Observatorium Series I, 186, 1-20 (1954)

395. Babcock, H.W.: The rotation of the Andromeda Nebula. Lick Obs. Bull. 19 (498), 41-51 (1939)

396. Rubin, V.: Rotational Properties of 21 Sc Galaxies with a Large Range of Luminosities and Radii from NGC $4605(\mathrm{R}=4 \mathrm{kpc})$ to UGC $2885(\mathrm{R}=122 \mathrm{kpc})$. Astrophys. J., 238, 471$487(1980)$

397. Ostriker, J.P., Peebles, J.P.E.: A Numerical Study of the Stability of Flattened Galaxies: or, can Cold Galaxies Survive? Astrophys. J., 186, 467-480 (1973)

398. Ostriker, J.P., Peebles, J.P.E., Yahil, A.: The size and mass of galaxies, and the mass of the universe. Astrophys. J. Lett., 193, L1-L4 (1974)

399. Bell, F.B., McIntosh, D.H., Katz, N., Weinberg, M.D.: A First Estimate of the Baryonic Mass Function of Galaxies. Astrophys. J. Lett., 585, L117-L120 (2003)

400. Turner, M.S.: The Case for $\Omega_{M}=0.33 \pm 0.035$. Astrophys. J., 576, L101-L104 (2002) 401. White, S.D.M., Rees, M.J.: Core condensation in heavy halos - A two-stage theory for galaxy formation and clustering. Mon. Not. R. Astron. Soc., 183, 341-358 (1978)

402. Battaner, E., Florido, E.: The Rotation Curve of Spiral Galaxies and its Cosmological Implications. Fund. Cosmic Phys., 21, 1-154 (2000)

403. López-Corredoira, M., Beckman, J.E., Casuso E.: High-velocity clouds as dark matter in the Local Group. Astron. Astrophys., 351, 920-924 (1999)

404. López-Corredoira, M., Betancort-Rijo, J., Beckman, J.E.: Generation of galactic disc warps due to intergalactic accretion flows onto the disc. Astron. Astrophys., 386, 169-186 (2002)

405. McGaugh, S.S.: Boomerang Data Suggest a Purely Baryonic Universe. Astrophys. J. Lett., 541, L33-L36 (2000)

406. Evans, N.W.: No Need for Dark Matter in Galaxies? In: Spooner, N.J.C., Kudryavtsev,

V. (eds.) Proceedings of the 3rd International Workshop on the Identification of Dark Matter, pp. 85-92. World Scientific, Singapore (2001)

407. Tasitsiomi, A.: The State of the Cold Dark Matter Models on Galactic and Subgalactic Scales. Int. J. Mod. Phys. D, 12(7), 1157-1196 (2003)

408. Sellwood, J.A.: Bar-Halo Friction in Galaxies. III. Halo Density Changes. Astrophys. J., 679, 379-396 (2008) 
409. Gnedin, O.Y., Kravtsov, A.V., Klypin, A.A., Nagai, D.: Response of Dark Matter Halos to Condensation of Baryons: Cosmological Simulations and Improved Adiabatic Contraction Model. Astrophys. J., 616, 16-26 (2004)

410. Di Cintio, A., Brook, C.B., Macciò, A.V., Stinson, G.S., Knebe, A., Dutton, A.A., Wadsley, J.: The dependence of dark matter profiles on the stellar-to-halo mass ratio: a prediction for cusps versus cores. Mon. Not. R. Astron. Soc., 437, 415-423 (2014)

411. Binney, J., Gerhard, O., Silk, J.: The dark matter problem in disc galaxies. Mon. Not.

R. Astron. Soc. 321, 471-474 (2001)

412. Casuso E., Beckman J.E.: On the Origin of the Angular Momentum of Galaxies: Cosmological Tidal Torques and Coriolis Force. Mon. Not. R. Astron. Soc., 449, 2910-2918 (2015)

413. McGaugh, S.: The Third Law of Galactic Rotation. Galaxies, 2, 601-622 (2014)

414. D'Onguia, E., Lake, G.: Cold Dark Matter's Small-Scale Crisis Grows Up. Astrophys. J., 612, 628-632 (2004)

415. Kroupa, P., Famaey, B., de Boer, K. S., et al.: Local-Group tests of dark-matter concordance cosmology . Towards a new paradigm for structure formation. Astron. Astrophys., 523, id. A32, 22 pp. (2010)

416. Kroupa, P., Theis, C., Boily, C.M.: The great disk of Milky-Way satellites and cosmological sub-structures. Astron. Astrophys., 431, 517-521 (2005)

417. Pawlowski, M.S., Kroupa, P.: The rotationally stabilized VPOS and predicted proper motions of the Milky Way satellite galaxies. Mon. Not. R. Astron. Soc., 435, 2116-2131 (2013)

418. Kroupa, P.: The Dark Matter Crisis: Falsification of the Current Standard Model of Cosmology. Publ. Astron. Soc. Australia, 29, 395-433 (2012)

419. López-Corredoira, M., Kroupa, P.: The Number of Tidal Dwarf Satellite Galaxies in Dependence of Bulge Index. Astrophys. J., 817, id. 75, 7 pp. (2016)

420. Lasserre, T., Afonso, C., Albert, J.N., et al.: Not enough stellar mass Machos in the Galactic halo. Astron. Astrophys., 355, L39-L42 (2000)

421. Moore, B.: Evidence against dissipation-less dark matter from observations of galaxy haloes. Nature, 370, 629-631 (1994)

422. Moore, B.: An upper limit to the mass of black holes in the halo of the galaxy. Astrophys. J. Lett., 413, L93-L96 (1993)

423. Sadoulet, B.: Deciphering the nature of dark matter Reviews of Modern Physics, 71, S197-S204 (1999)

424. Aharonian, F., Akhperjanian, A.G., Bazer-Bachi, A.R., et al.: The H.E.S.S. Survey of the Inner Galaxy in Very High Energy Gamma Rays. Astrophys. J., 636, 777-797 (2006)

425. Sánchez-Conde, M.A.: Gamma-ray dark matter searches in the Milky Way. Paper presented at the Conference Distribution of Mass in the Milky Way, Leiden, Netherlands, 13-17 July 2009

426. Toomre, A.: What amplifies the spirals. In: Fall, S.M., Lynden-Bell, D. (eds.) The Structure and Evolution of Normal Galaxies, pp. 111-136. Cambridge University Press, Cambridge (U.K.) (1981)

427. Sanders, R.H., McGaugh, S.S.: Modified Newtonian Dynamics as an Alternative to Dark Matter. Ann. Rev. Astron. Astrophys., 40, 263-317 (2002)

428. Drexler, J.: Identifying Dark Matter Through the Constraints Imposed by Fourteen Astronomically Based 'Cosmic Constituents'. arXiv.org: astro-ph/0504512 (2005)

429. Mayer, F.J., Reitz, J.R.: Electromagnetic Composites at the Compton Scale. Int. J. Theor. Phys., 51, 322-330 (2012)

430. Hajdukovic, D.S.: Virtual gravitational dipoles: The key for the understanding of the Universe? Physics of the Dark Universe, 3, 34-40 (2014)

431. Padmanabhan, T.: Cosmological constant-the weight of the vacuum. Phys. Reports, 380, 235-320 (2003)

432. Fukugita, M., Lahav, O.: Ly-alpha clouds at low redshift and the cosmological constant. Mon. Not. R. Astron. Soc., 253, 17P-20P (1991)

433. Longair, M.S.: Observational Cosmology 1986. In: Hewitt, A., Burbidge, G., Fang, L.Z. (eds.) Observational Cosmology (IAU Symp. 124), pp. 823-840. Reidel, Dordrecht (1987) 434. Efstathiou, G., Sutherland, W.J., Maddox, S.J.: The cosmological constant and cold dark matter. Nature, 348, 705-707 (1990) 
435. Aguirre, A., Haiman, Z.: Cosmological Constant or Intergalactic Dust? Constraints from the Cosmic Far-Infrared Background. Astrophys. J., 532, 28-36 (2000)

436. Goobar, A., Bergström, L., and Mörtsell, E.: Measuring the properties of extragalactic dust and implications for the Hubble diagram. Astron. Astrophys., 384, 1-10 (2002)

437. Milne, P.A., Foley, R.J., Brown, P.J., Narayan, G.: The Changing Fractions of Type Ia Supernova NUV-Optical Subclasses with Redshift. Astrophys. J., 803, id. 20, 15 pp. (2015)

438. Knop, R.A., Aldering, G., Amanullah, R., et al.: New Constraints on $\Omega_{M}, \Omega_{\Lambda}$, and $w$ from an Independent Set of 11 High-Redshift Supernovae Observed with the Hubble Space Telescope. Astrophys. J., 598, 102-137 (2003)

439. Rowan-Robinson, M.: Do Type Ia supernovae prove $\Lambda>0$ ? Mon. Not. R. Astron. Soc., $332,352-360(2002)$

440. Shanks, T., Allen, P.D., Hoyle, F., Tanvir, N.R.: Cepheid, Tully-Fisher and SNIa Distances. arXiv.org: astro-ph/0102450 (2001)

441. Domínguez I., Höflich P., Straniero O., Wheeler C. Evolution of type Ia supernovae on cosmological time scales. Mem. Soc. Astron. Ital., 71, 449-460 (2000)

442. Howell, D.A., Sullivan, M., Nugent, P.E., et al.: The type Ia supernova SNLS-03D3bb from a super-Chandrasekhar-mass white dwarf star. Nature, 443, 308-311 (2006)

443. Quimby, R., Höflich, P., Craig Wheeler, J.: SN 2005hj: Evidence for Two Classes of Normal-Bright SNe Ia and Implications for Cosmology. Astrophys. J., 666, 1083-1092 (2007)

444. Podsiadlowski, P., Mazzali, P.A., Lesaffre, P., Wolf, C., Forster, F.: Cosmological Implications of the Second Parameter of Type Ia Supernovae. arXiv.org: astro-ph/0608324 (2006)

445. Shu, W.-Y.: The Geometry of the Universe. arXiv.org: 1007.1750 (2010)

446. Romano, A.E.: Lemaitre-Tolman-Bondi universes as alternatives to dark energy: Does positive averaged acceleration imply positive cosmic acceleration? Phys. Rev. D, 75(4), id. 043509 (2007)

447. Vishwakarma, R.G., Narlikar, J.V.: Modeling Repulsive Gravity with Creation. J. Astrophys. Astr., 28, 17-27 (2007)

448. Oliveira, F.J., Hartnett, J.G.: Carmeli's Cosmology Fits Data for an Accelerating and Decelerating Universe Without Dark Matter or Dark Energy. Foundations of Physics Letters, 19(6), 519-535 (2006)

449. Thompson, R.I.: Constraints on quintessence and new physics from fundamental constants. Mon. Not. R. Astron. Soc., 422, L67-L71 (2012)

450. Jackson, J.C., Dodgson, M.: Deceleration without dark matter. Mon. Not. R. Astron. Soc., 285, 806-810 (1997)

451. Weinberg, S.: The cosmological constant problem. Rev. Mod. Phys., 61, 1-23 (1989)

452. Unzicker, A.: Vom Urknall zum Durchknall. Die absurde Jagd nach der Weltformel. Springer, Heidelberg (2010)

453. Melia, F., Shevchuk, A.S.: The $R_{h}=c t$ universe. Mon. Not. R. Astron. Soc., 419, 2579-2586 (2012)

454. Mitra, A.: Why Friedmann cosmology cannot describe the observed universe having pressure and radiation. J. Mod. Phys., 2, 1436-1442 (2011)

455. Constantin, A., Shields, J.C., Hamann, F., Foltz, C.B., Chaffee, F.H.: Emission-Line Properties of $z>4$ Quasars. Astrophys. J., 565, 50-62 (2002)

456. Iwamuro, F., Motohara, K., Maihara, T., Kimura, M., Yoshii, Y., Doi, M.: Fe II/Mg

II Emission-Line Ratios of QSOs within $0<z<5.3$. Astrophys. J., 565, 63-77 (2002)

457. Dietrich, M., Hamann, F., Appenzeller, I., Vertergaard, M.: Fe II/Mg II Emission-Line Ratio in High-Redshift Quasars. Astrophys. J., 596, 817-829 (2003)

458. Freudling, W., Corbin, M.R., Korista, K.T.: Iron Emission in $z \sim 6$ QSOS. Astrophys. J. Lett., 587, L67-L70 (2003)

459. Maiolino, R., Juarez, Y., Mujica, R., Nagar, N., Oliva, E.: Early Star Formation Traced by the Highest Redshift Quasars. Astrophys. J. Lett., 596, L155-L158 (2003)

460. Barth, A.J., Martini, P., Nelson, C.H., Ho, L.C.: Iron Emission in the $z=6.4$ Quasar SDSS J114816.64+525150.3. Astrophys. J. Lett., 594, L95-L98 (2003)

461. Sardane, G.M., Turnshek, D.A., Rao, S.M.: Ca II absorbers in the Sloan Digital Sky Survey: statistics. Mon. Not. R. Astron. Soc, 444, 1747-1758 (2014) 
462. Dunne, L., Eales, S., Ivison, R., Morgan, H., Edmunds, M.: Type II supernovae as a significant source of interstellar dust. Nature, 424, 285-287 (2003)

463. Castro-Rodríguez, N., López-Corredoira, M.: The age of extremely red and massive galaxies at very high redshift. Astron. Astrophys., 537, id. A31, 6 pp. (2012)

464. Longhetti, M., Saracco, P., Severgnini, P., et al.: Dating the stellar population in massive early-type galaxies at $z$ 1.5. Mon. Not. R. Astron. Soc., 361, 897-906 (2005)

465. Trujillo, I., Feulner, G., Goranova, Y., et al.: Extremely compact massive galaxies at $z \sim$ 1.4. Mon. Not. R. Astron. Soc. 373, L36-L40 (2006)

466. Labbé, I., Huang, J., Franx, M., et al.: IRAC Mid-Infrared Imaging of the Hubble Deep Field-South: Star Formation Histories and Stellar Masses of Red Galaxies at $z>2$. Astrophys. J., 624, L81-L84 (2005)

467. Toft, S., van Dokkum, P., Franx, M., Thompson, R.I., Illingworth, G.D., Bouwens, R.J., Kriek, M.: Distant Red Galaxies in the Hubble Ultra Deep Field. Astrophys. J., 624, L9-L12 (2005)

468. Rodighiero, G., Cimatti, A., Franceschini, A., Brusa, M., Fritz, J., Bolzonella, M.: Unveiling the oldest and most massive galaxies at very high redshift. Astron. Astrophys., 470, 21-37 (2007)

469. Wiklind, T., Dickinson, M., Ferguson, H.C., Giavalisco, M., Mobasher, B., Grogin, N.A., Panagia, N.: A Population of Massive and Evolved Galaxies at $z>\sim 5$. Astrophys. J., 686, 781-806 (2008)

470. Steinhardt, C. L., Capak, P., Masters, D., Speagle, J.S.: The impossible early galaxy problem. Astrophys. J., 824, id. 21, 9pp. (2016)

471. Guo, Q., White, S., Boylan-Kolchin, M., et al.: From dwarf spheroidals to cD galaxies: simulating the galaxy population in a $\Lambda$ CDM cosmology. Mon. Not. R. Astron. Soc., 413, 101-131 (2011)

472. Pérez-González, P.G., Rieke, G.H., Villar, V., et al.: The Stellar Mass Assembly of Galaxies from $z=0$ to $z=4$ : Analysis of a Sample Selected in the Rest-Frame NearInfrared with Spitzer. Astrophys. J., 675, 234-261 (2008)

473. Riechers, D.A., Bradford, C.M., Clements, D.L., et al.: A dust-obscured massive maximum-starburst galaxy at a redshift of 6.34. Nature, 496, 329-333 (2013)

(c) 2017 by the authors; licensee Preprints, Basel, Switzerland. This article is an open access article distributed under the terms and conditions of the Creative Commons Attribution (CC BY) license http://creativecommons.org/licenses/by/4.0/). 